\title{
MORTAL INFILL
}

Finding space for the dead in today's suburbs

By

Martin Neil Smith Powell

$$
\text { A thesis }
$$

submitted to the Victoria University of Wellington

in partial fulfilment of the requirements for a degree of

Master of Landscape Architecture 



\section{Abstract}

The spaces set aside for the dead in contemporary cities have been shifted to the periphery. As a result the built fabric of

today's suburbs does little to acknowledge the mortality of their inhabitants.

This thesis aims to introduce spaces for the dead into these suburbs through a network of memorial walls, known as columbaria', which will provide inhabitants with the option of interment closer to their own communities.

A series of design principles have been developed, through research and design testing, for the distribution and composition of these spaces. This testing has been carried out through a design case study, focusing on site selection and design, based in Wellington city.

The resulting design principles provide a framework for the establishment of a place for death within contemporary suburbs, increasing their inhabitants' awareness of their own mortality, and consequently their appreciation of life. 



\section{Acknowledgements}

As this thesis is concerned with the commemoration of the dead, It seems appropriate to firstly thank both of my late Grandfathers; Neil

for the critical thinking which has allowed me to complete my studies, and Mick for the passion for cricket which has kept me sane whilst doing so.

Thanks to Victoria University of Wellington, both for providing me with so many great opportunities through my studies, but also for the Masters by Thesis Scholarship which has allowed me to focus on research rather than financial worries this year.

Thanks to my supervisor; Chris McDonald; whose regular feedback and encouragement has been incredibly valuable.

Thanks to the Southern Cross Garden Bar \& Restaurant for both employment and abundant caffeine when necessary.

Thanks to my studio colleagues; whose advice, humour and encouragement I could not have gone without.

And finally thanks to my mother, father, sister and friends for their support and understanding throughout my year in hiding. 


\section{Glossary of Terms}

Columbarium A wall containing niches for the interment of ashes.

Columbaria The Plural form of Columbarium.

Churchyard A small burial area associated with a nearby church. Generally found at a settlement's centre.

Cemetery A larger scale burial area generally found at the settlement's edge when first constructed.

Interment The act of disposing of/storing human remains 


\section{Table of Contents}

\section{Abstract}

Acknowledgements

Glossary of Terms

INTRODUCTION

Problem

Aim

Scope

Significance

Overview

Design Principles

Context Review

Introduction

Historical Context

Theoretical Context

Cultural Context

Approaches

Conclusions
Precedents

Introduction

De Nieuwe Ooster Columbarium

Friedhof Hörnli Columbarium

Friedhof Steckborn Columbarium

Westminster Urban Columbarium

Arradon Columbarium

Conclusions

\section{Design PrinCIPLES}

Introduction

Informed Introduction

Obvious Improvement

Infill Impacts

Layered Intimacy

Individualised Interment

Conclusions

\section{Site Selection}

Introduction

City Scale Network - Existing

City Scale Network - Proposal

Conclusions
DESIGN

Introduction

Brooklyn - Catalyst

Kilbirnie - Development Alignment

Conclusions

EXEGESIS

\section{Introduction}

General Design Discussion

Informed Introduction Discussion

Obvious Improvement Discussion

Infill Impacts Discussion

Layered Intimacy Discussion

Individualised Interment Discussion

Synthesis of Principles

Conclusions
3 



\section{INTRODUCTION}


The space allocated for the dead in the contemporary city is 1 a topic subject to much discussion across a wide variety of disciplines, from sociology to history. However spatial design disciplines such as Landscape Architecture and Urban Design in terms of propositions for spaces other than the city-scale cemetery now seen as the norm in most western cities.

In one of the few architectural discussions of these spaces Last Landscapes: The Architecture of the Cemetery in the West Ken Worpole identifies the need for these propositions, stating that:

“...unless planners, architects and landscape designers take seriously the issue of how to create new kinds of cemeteries ... there is a real danger of creating cities without memory, cities in denial of death and humanity." (31)

As such this thesis seeks to investigate this topic through Landscape Architecture and Urban Design.

\section{Problem}

Traditional settlement patterns have generally involved a space allocated for the dead close to the community's centre. These spaces were generally experienced on an everyday basis by the town's inhabitants. The town churchyard is a commonly referenced example of this.

However when cities of considerable density have developed, such as the cities of the west following the industria revolution, the spaces allocated for the dead have been pushed to the periphery. These spaces are more difficult to reach and are seldom experienced on a frequent basis by the communities for whom they are built. This is particularly true in today's cities, where the periphery is often hours of travel from the places where the majority of people live.

Ultimately the built fabric of today's suburbs does little to acknowledge the mortality of their own inhabitants.

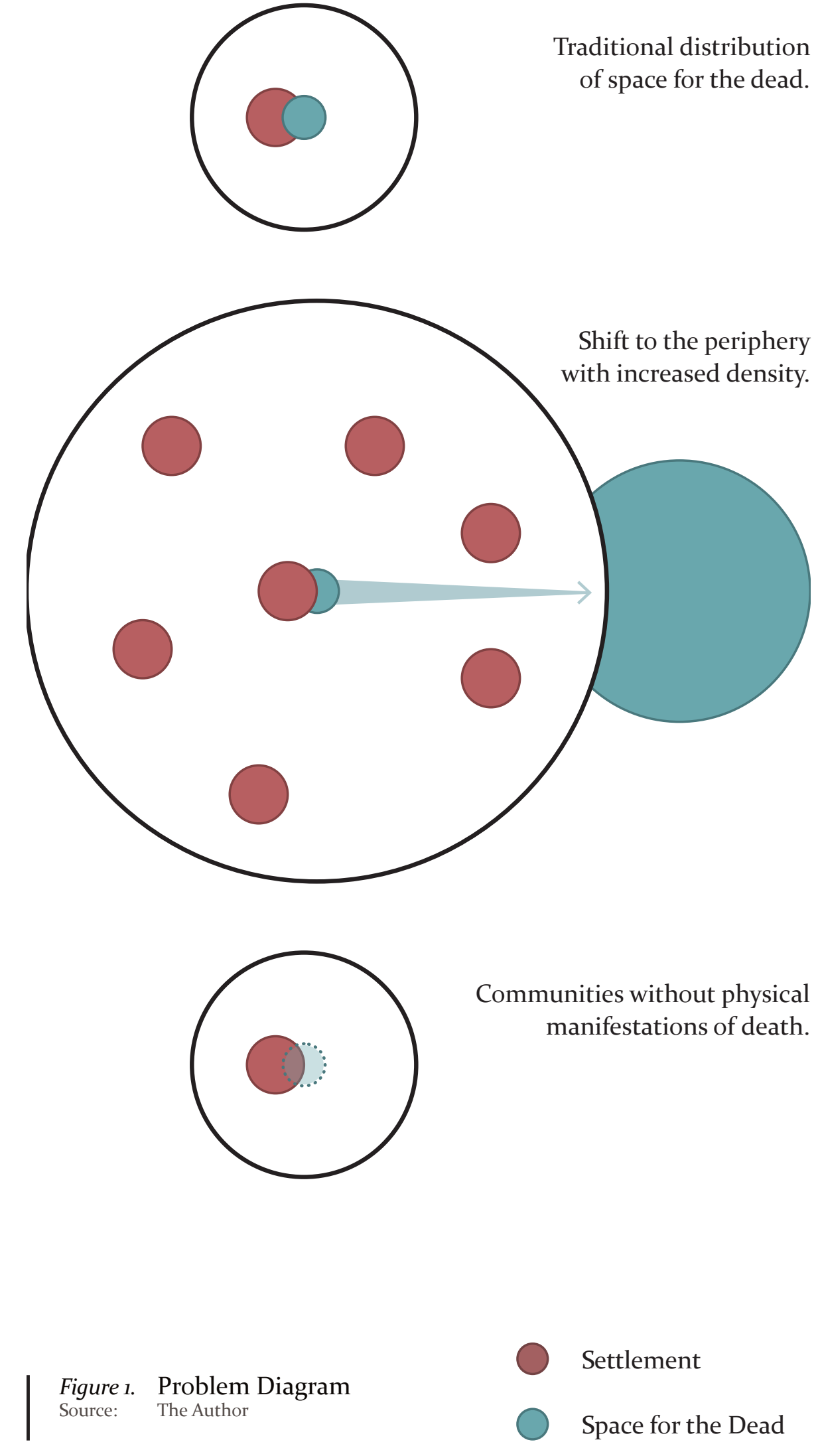




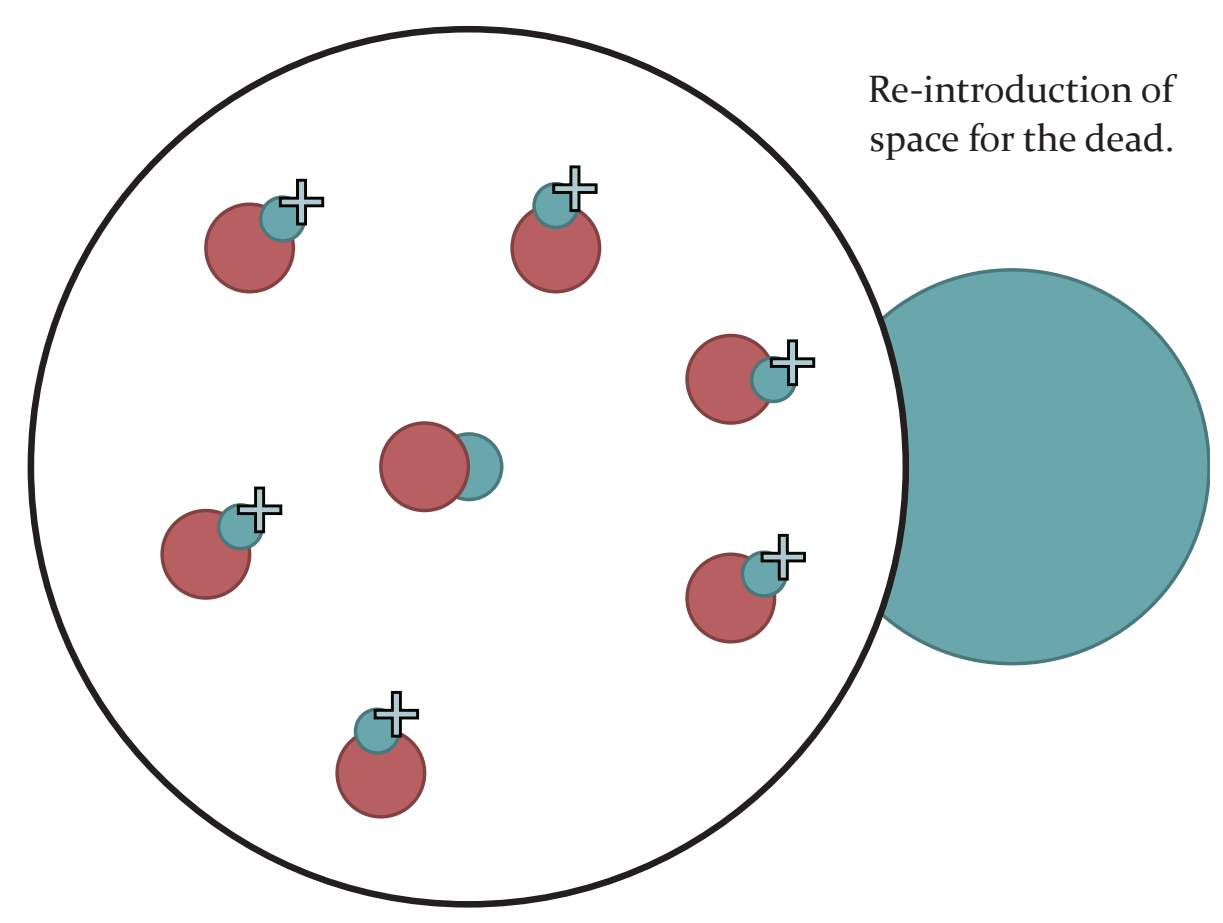

Aim

\section{Scope}

The proposition investigated in this thesis is:

\section{To find space for the dead within today's suburbs.}

More specifically it aims to investigate existing suburbs in Wellington city. Because these suburbs are existing rather than blank green fields sites, the spatial footprint that can be worked with is somewhat smaller.
Between $70 \%$ and $80 \%$ of New Zealanders choose to be cremated(Raudon), as such the thesis focuses on options for

This proposition does not argue for the replacement of the existing centralised system of large cemeteries, but rather to augment it with a series of 'satellite' spaces for the dead located close to their associated communites.

As practices around death are often the product of cultural and religious beliefs, the death practices of New Zealand's common cultures and faiths has been reviewed in order to work from a more informed understanding of the country's present day diversity. However most of these communities are spread across entire cities and as such require a centralised space rather than one tied to any one particular suburb.

Instead the proposition looks to provide a religiously/ culturally non-specific option, seeking to cater to those with a strong connection to a specific suburban community rather than a specific faith or culture. This also aligns with New Zealand's increasingly secular population. (Roudon) 


\section{Significance}

This thesis provides an example or case study for those carrying out more in-depth theoretical research on the topic of spaces for the dead.

The design principles produced and refined throughout the design/research process could have practical use for those working on similar real world projects involving spaces for the dead.

Planning, policy and design professionals may also find the processes used helpful in making a proposition for the introduction of controversial land uses.

Also, as a review of the Burial and Cremation Act (1964) by the Law Commission is currently underway (Brett), the thesis has the potential to contribute to the larger contemporary discussion surrounding the future of New Zealand's attitudes towards death and spaces for the dead.

\section{Methodology}

The design research for this thesis has not been linear. While an initial background was established at the beginning of the process, further background material has been uncovered as new problems have presented themselves throughout the process.

The design and research aspects of the thesis are therefore strongly interrelated. However for the sake of clarity the thesis has been split into background, proposition and discussion.

The background consists of a Context Review (II) chapter consisting of historical, theoretical and cultural sections, and a Precedent Studies (III) chapter reviewing contemporary approaches to the siting and design of columbaria walls.

The proposition begins with a Design Principles (IV) chapter detailing the principles which have been used throughout the proposal. A chapter is then devoted to Site Selection (V) within six of Wellington's Suburbs. This leads to the Design (VI) taking two of these sites and developing design solutions for each.

The discussion is found in the final chapter, an Exegesis (VII) of both the design principles and their application to Wellington City

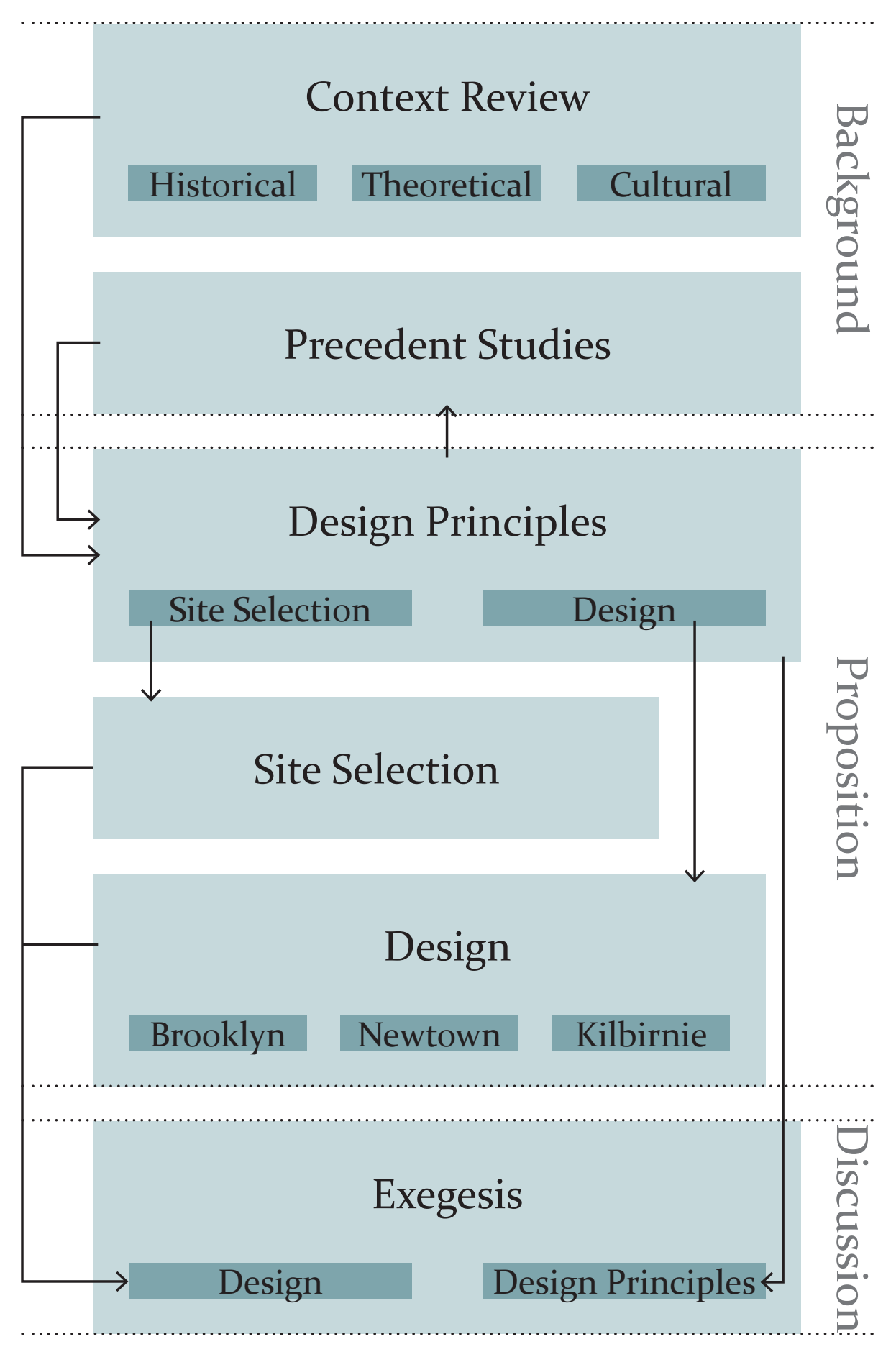

Figure 3. Thesis Method Diagram 
Informed Introduction

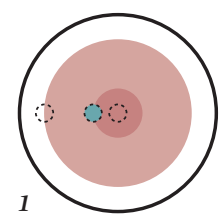

Aligning space for the dead with the community's core without undermining its existing significance to the community.

\section{Obvious Improvement}

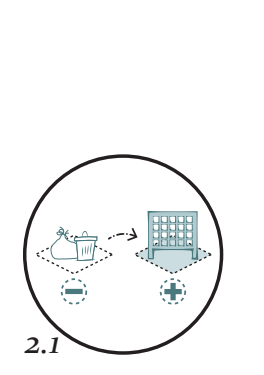

Selecting underutilised sites where designed intervention will be an
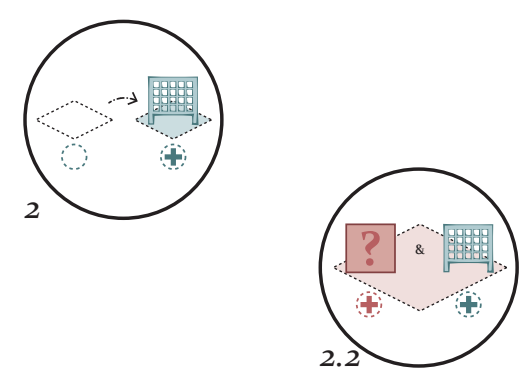

Integrate other land uses which are with the space for the dead.

Infill Impacts
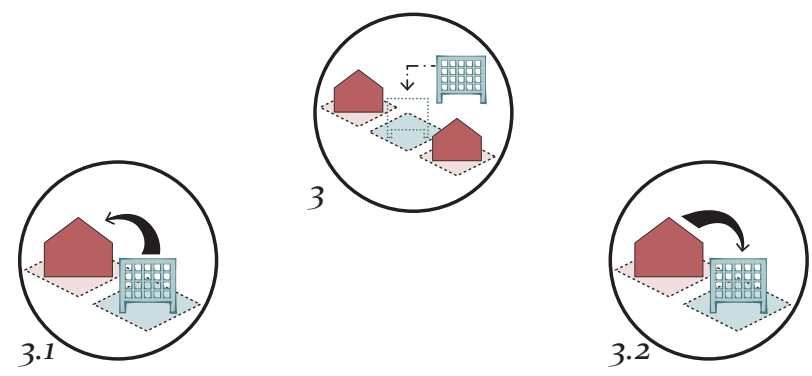

Taking into account the impacts surrounding land uses... obvious improvement.

\section{Layered Intimacy}

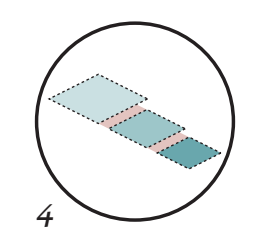

Establish a sequence of distinctive spaces, separated by clear thresholds, leading from public to intimate and concluding with a space for ceremony and

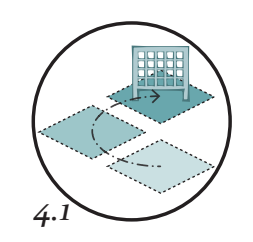

Distinctive Spaces interment.

\section{Individualised Interment}

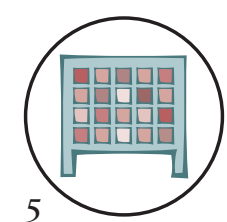

Allow for individualisation within the commemorative spaces without compromising the design's overall integrity.

\section{Design Principles}

While the principles have developed from ideas to normative spatial propositions throughout the background chapters, a summary of them is given here in order to better illustrate how they evolve throughout the thesis.

Throughout the thesis these symbols are used to notate when the specific idea behind each principle is being discussed. In the core chapters they are used to indicate where design principles are being applied. Within the exegesis each of these ideas serves as a section of the chapter, where that aspect of both the design and the principle itself is critically assessed. ....and the impact of these land uses
on the space for the dead when on the space for the dead when 

Context Review 



\section{Introduction}

n order to understand why the built fabric of today's suburbs 1 do so little to acknowledge their inhabitants mortality, the historical, theoretical and cultural context of spaces built to commemorate the dead must first be addressed.

This is a rich subject with many debates ranging through a variety of subtopics. However due to the limited scope of the thesis, those of specific relevance to the aim of the thesis have been the primary focus. Insights gained from these discussions are intended to contribute directly to the development of the design principles.

This chapter achieves this by first examining the history of spaces or the dead, focusing on their distribution in relation to settlement and the spatial practices of their associated communities.

Following this, theoretical perspectives relevant to the topic are addressed. The issue of society's 'death taboo' is discussed, followed by a look at the prominent approaches to memorial last century, particularly following World War II.
Next the specific cultural context of New Zealand is examined with regards to the problem, in particular the growing prominence of issues around death in an aging society, the varied beliefs existing in a multicultural country, and the specific 'kiwi' culture of death.

Finally, the propositions made by Christopher Alexander in his book A Pattern Language regarding 'grave sites' are discussed as an example of previous approaches to the problem of finding spaces for the dead in the places where we live. 


\section{Historical Context}

The shift of space for the dead from a community's centre to the periphery is a reoccurring change often tied to settlement density One of the first societies to experience this shift was that of $3000 B C-2000 B C$ Mesopotamian civilisation in the modern day country of Iraq

Earlier settlements of a relatively small size within this civilization were characterised by spaces for the dead located at the centre. In describing the settlement of Kish, Torres-Rouff, Pestle and Daverman point out: "Although failing to conform to modern norms defining cemeteries as removed places used exclusively for disposing of the dead, the ... residential burials, made in such close proximity to living spaces, would have served to exten and strengthen ties between the living and the dead." (199) They argue that these ties are important to the formation of a collective identity for the relatively young civilisation. (200) However later records of the larger city of Ur indicate that spaces for the dead had been moved to the edge of the city. (Wilford) (McIntosh 224)

The transition of ancient Greek city states to the classical period also saw a similar shift of space for the dead from settlement centre to periphery. The main spaces for the dead in
Athens were located within the city limits at Kerameikos and Agora Archaic cemetery. (Morris 21) However as the city's density increased this changed: by "... c. 500 BC new cemeteries seem to begin outside ... the city gates." (22)

This explicit border of the cities edge as a line between space for the living and the dead is perhaps best exemplified in Rome where laws "...forbade burial or cremation within the city walls (pomerium), and led over time to the establishment of loca religiosa (formal cemeteries)."(Retief 134) These 'loca' also held some of the first columbaria which "...during the period under discussion [c.80o $B C-c .100 A D$ were] the preferred procedure [for interment]."(135)

It is clear that this shift of space for the dead from settlements centres to their periphery is not solely a modern process, but has, in fact, occurred in many situations where population density has risen to a certain level.

Since the fall of the Roman Empire and the rise of Christianity in the west, however, settlement has occurred primarily with the space for the dead at the centre. The population densities which had existed were for the most part dispersed, leading to lower density settlements and contributing to the shift of these spaces back to the centre of settlement. The 
formation of a collective identity, cited as a reason for the earlier central spaces for the dead in Kish (Torres-Rouff, Pestle and Daverman), may also have played a role in this shift in the Christian world, as the religion was still in its relative infancy.

The pre-industrial revolution landscape of mortality was "... largely comprised [of] small-scale graveyards owned and managed by the established church and usually surrounding the church itself." (Rugg "Lawn Cemeteries" 215) In essence the placement of the space for the dead was bound to the placement of the actual

church. It follows that, as the church was a community centre, the churchyard was generally found directly adjacent to the church, at the centre of settlement

The churchyard had a largely informal character, seldom being the product of a specific 'design' and was small in scale (Basmajian and Coutts 306) allowing for a sense of intimacy within what is still effectively a 'public' space. It has become the space for the dead associated with lower density settlement in the western world.

The industrial revolution brought about many dramatic changes in society, not the least of which was a dramatic

increase in population densities. The churchyards, which had been established to accommodate lower population densities of preindustrial settlement, quickly filled leaving a demand for further space for burial. (Rugg "A Few Remarks on Modern Sepulture" 116) The prevailing response was to find a large tract of readily available land "...perhaps half a mile away..." from the settlement's edge. (Rugg "Defining the Place of Burial" 261) Perhaps the most discussed example of this is Paris, where the overcrowding of churchyards was particularly severe. "In 1765 the Parliament of Paris published a decree that proposed to move burial from the heart of the city to its periphery..." "A Few Remarks on Modern Sepulture" 114)

The fact that early cemeteries were generally built at the periphery is perhaps not clear on first consideration. Most have been absorbed by the urban fabric as suburbs developed around and past them. In many cases they have become a central part of the suburb, and are now described as "urban cemeteries" (Odland 44). However their origins are still generally that of a large tract of land at the periphery of a settlement.

A well-known example of this is Père-Lachaise. When it was first established (at the behest of the previously mentioned decree) by urban planner Nicolas Frochot in 1804 it was almost $2 \mathrm{~km}$ from the city's edge, and was deemed too far by the Parisian 
population, seeing very few burials in its first ten years. (paris.fr) However as the city expanded it quickly became one of Paris' most central cemeteries and an integral part of the $20^{\text {th }}$ arrondissement. It was fiercely defended by the same Parisian population when its relocation was proposed by Baron Haussmann later that century. (Odland 41)

As cities have grown larger, so too have the populations the cemeteries are required to serve. While the first cemeteries were large in comparison to the churchyards which preceded them,

the ever increasing growth of urban populations meant that these spaces were, in turn, filled. Of course the response to this was to once again find a large space tract of readily available land.

However with the suburbanisation that occurred in many cities in the latter half of last century, the edge of the city is no longer the edge of a specific residential settlement, and instead of a twenty minute trip to the cemetery, up to a two hour commute is now required. This has led to what Worpole describes as "... severing the geographical (and anthropological) link between the community of life and the community of death." (31)

In addition these modern cemeteries are built to cater to communities of hundreds of thousands rather than thousands, leading critics such as Aaron Odland to argue that "... a cemetery of decent size tends to be more the scale of a regional park than a neighbourhood park." (41) Worpole argues that in this adjustment of scales the nuances of culture have often been passed over and that "Many modern cemeteries seem to be designed in the spirit of mass production..." (56) This change in scale is more than just a difference in acres; it has a profound impact on the intimacy of the spaces.

While in many cases the old churchyard would be retained, in others the dead would literally be moved to the new sites in order to allow development on that land. Odland uses the example of a former Jewish Burial Ground near his apartment in San Francisco: "The land was purchased by the city of San Francisco just before the earthquake of 1908, and the bodies were exhumed and relocated to Colma, a suburb just south of the city. This happened to most of the burial sites in San Francisco during that same era, with city expansion ... justifying the exchange of use."(40) This, combined with San Francisco's only land border being to the south, has led to Colma having 1.5 million dead across 17 cemeteries despite having a living population of only 1,191. (Brennan) 


\section{Theoretical Context}

A question which emerges is to what extent is this actually a problem? At the centre of this question is the premise that these spaces are, as Worpole argues, "... a vital part of the urban pallet of public institutions and public open space..."

A common argument is that this shift of spaces for the dead to the periphery is a symptom of a larger societal denial of death. It has been commonly put forward that, since the end of the Victorian era (which was noticeably fixated with death) (Rugg "A Few Remarks on Modern Sepulture" 119) death has effectively become another taboo within Western Society. In 1915 Sigmund Freud stated that "We showed an unmistakable tendency to put death on one side, eliminate it from life. We tried to hush it up" (77-78) While more recently Curl has associated changes in spaces for the dead to "...an irrational desire to deny that death and its realities have any place in the modern world" (360) The lack of a daily reminder of mortality via a space allocated for the dead could certainly be attributed to these attempts to 'hush death'.

However the actual existence of this death taboo is debated by several contemporary authors. Worpole states that: "I do not subscribe to the commonly expressed view that modern societies have 'abolished' death or hidden it from view."(8) It has been argued that, if death was such a taboo, it is "... a very badly kept secret; such that there are over 750 books now in print asserting that we are ignoring the subject." (Tarlow 6) Indeed the proposition the the is is somehow 'hidden' within contemporary soc a difficult one to accept when consider withing the exposure to death found in film, television and news media alone.

However this does not necessarily debase the importance of spaces for the dead as reminders of mortality. In many cases these other reminders of death, while present, are easily dismissed as exterior to oneself, whereas experiencing an actual physical manifestation of death, in particular the presence of human remains, can serve as a much stronger reminder of the universality of human mortality. Worpole points out that the media, in particular film and television, portray the cemetery more often than the landscape does and that the 'fictional' cemetery has a far stronger presence in the contemporary psyche than that of the actual space for the dead experienced on a daily basis (8). As such he concludes that "... While I do believe that most people still treat death seriously, I do think that in its topographical, processional, landscaping and architectural aspects, commemoration has been poorly served in recent times." 
This debate over the 'death taboo' played an important ole in discussion surrounding approaches to commemorative memorials throughout last century. This focused primarily on the War Memorials of the United States, however it can be applied to most western civilisations, particularly those which participated in the 'World Wars'. At the centre of this discussion was the 'traditional memorial' (often described as 'dead') and the 'living memorial'.

Joseph Hudnut's 1945 book The Monument does not Remember represented one of the strongest voices in this argument, arguing that the traditional memorials would "...try to bring the grim business to your doorstep in a democratic guise. They will not succeed. The war will hide its head behind the common man quite as easily as behind the trophies of conquerors." (57) These traditional memorials were argued to be based on a symbolism no longer relevant to contemporary society. In many cases they were argued to have become "...symbols not of victory or of peace but of industrial capitalism's penchant for war, as well as its related inability to care for its public spaces and built environment." (Shanken 139) In many cases the traditional memorials were portrayed as monuments to the leaders and generals involved in wars (Forum) rather than the 'common man' mentioned by Hudnut. They were commonly associated with death, generally in a negative light. Shanken describes the rhetoric of one critic; Louis Bromfield, as such: "For Broomfield the appellation "dead" damned the traditional memorial, and he repeated it gleefully like a dirty word..." This seems to demonstrate, at least to some extent, an aversion to death similar to that discussed previously.

The alternative to these 'dead' memorials was the 'living memorial'. These memorials were to serve as practical and utilitarian land uses which would, by virtue of their use, commemorate the sacrifices of war. A 1945 edition of The Architectural Forum argued that "With the recognition that the price of victory had been paid by the group came a correlative

feeling that the war memorial might best commemorate the group sacrifice by increasing group welfare."(141) Lewis Mumford argued against this, stating that "... such a practical result cannot be achieved by an auditorium or a swimming pool, perfunctorily named a memorial. These useful structures do not bind us to the dead..." However, as Shanken points out, where these would have been "... a common sentiment during World War I; in 1945 [they] represented a shrinking minority."(132)

Interestingly much of this debate and discussion occurred during (not after) the Second World War. It is described as a ".. long and wide ranging wartime debate over memorials that for 
two years had filled the pages of art and architectural magazines, the popular press, trade and even scholarly journals."(Shanken 130) This is likely because for commemoration to be integrated in to public infrastructure the concept of 'living memorials' would have to be approved by the time post-war development began (which was almost immediately). In fact designs for specific memorial community centres were already featuring in magazines such as The Architectural Forum prior to the allied victory in the Pacific. (145)

The memorials which resulted from these discussions were, of course, far less polemic than the discussions surrounding them. (Shanken 130) Much of the time while the community centre or park would be named as a memorial, a smaller section of the development would also be devoted to a more traditional memorial. (Forum 147)

The concept of integrating other utilitarian land uses in to a memorial is an effective one, as it encourages continued community involvement with the memorial. (Shanken 133)

However the most effective of these also retained specific physical manifestations of this memorial, rather than abolishing them entirely as was argued by many of the traditional memorial's strongest critics. 



\section{Cultural Context}

The living memorial has also been made use of throughout New Zealand, though perhaps not quite as extensively. The war monument remained the most prominent mode of commemoration. So much so that NZS 4242, revised in 1995 , still includes a standard for an obelisk and a spire in its drawing specifications $(20,21)$. However there still exist a large number of memorial schools, parks and halls throughout the country. Smaller, more ad-hoc forms of memorial influenced by the "living memorial' concept have also increased significantly, in particular the tradition of memorial seats and fountains now found across the country. Similarly, the use of roadside crosses for accident victims can be seen as an extension of the traditional memorial which has become a commonplace commemoration of road deaths throughout the country.

The 'death taboo' also exists within New Zealand to a certain extent. Despite a being exposed to death in the media frequently we possess quite an aversion to the physical presence of death. A recent example of this is a crematorium proposed for Sydenham in Christchurch, which was met by strong opposition by the local community despite having all required consents. A quote from local MP Jim Anderton exemplifies the sentiment that a business associated with death, no matter how successful, will not be accepted if its placement isn't well considered: "This is an area which really needs revitalization, and plonking a crematorium in the middle of it doesn't have the right ring to it..." (Sachdeva) This makes clear the sensitivity of New Zealanders to the placement of spaces associated with death.

New Zealand has its own specific culture around death Sally Rauden has researched this by comparing practices in New Zealand, Norway, Sweden and Scotland. (Raudon "Angelboxes in the Land of Ikea") Though all countries with a similar level of secular beliefs, she found that New Zealand's practices were still significantly different, focusing far more on the individual. (Harvey) We have a tendency to customise ceremonies to reflect the personality of the deceased rather than follow a strict ritual. (Raudon "The Nz Way of Death") This desire for the opportunity to customise and individualise is a noteworthy aspect of our culture of death. The speed at which this tendency has developed also exemplifies that New Zealanders are relatively receptive to cultural changes surrounding mortality, as long as they are not too dramatic. 
However as much as there are a number of cultural tendencies which can be attributed to New Zealanders as a whole, our society is a multicultural one, and therefore our beliefs and practices around death are inherently multifaceted. This is best addressed in the book Last Words commissioned by the Funeral Directors Association of New Zealand and edited by Margot

Schwass. In this book the predominant cultures and faiths of New Zealand are described in relation to their practices around death. An analysis of this text has been performed focusing specifically on spatial practices of these communities in relation to spaces for the dead. (See Fig. 4)

Most notable is the fact that there are several cultures and faiths which are very much uncomfortable with cremation. For this reason any solution involving just cremation must act as an augmentation to the existing system of cemeteries rather than as a replacement. In addition the discomfort these comm as a reet with may feel with cremation must betaken in to account when spaces associated with death. Also of importance is that almost all groups have a preference for some form of committal ceremony. With this in mind, any space of commemoration would need to be able to accommodate these events, which are generally separate from the funeral and consist of a smaller group of close family and friends.

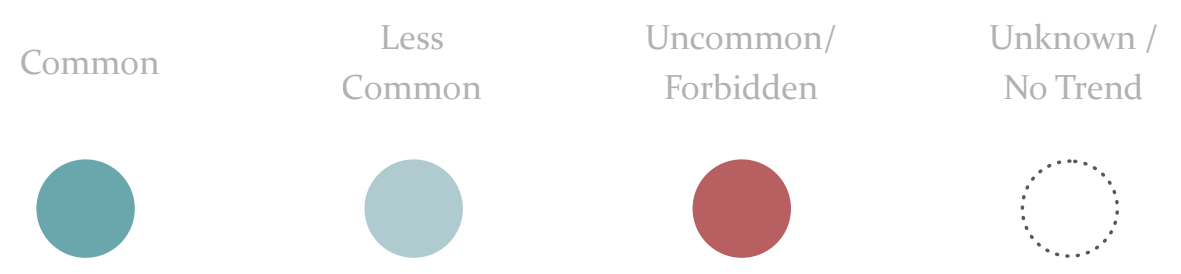

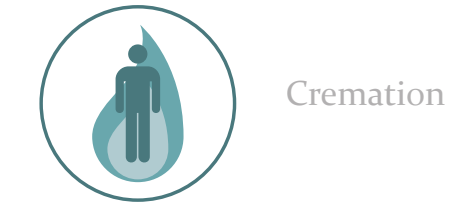
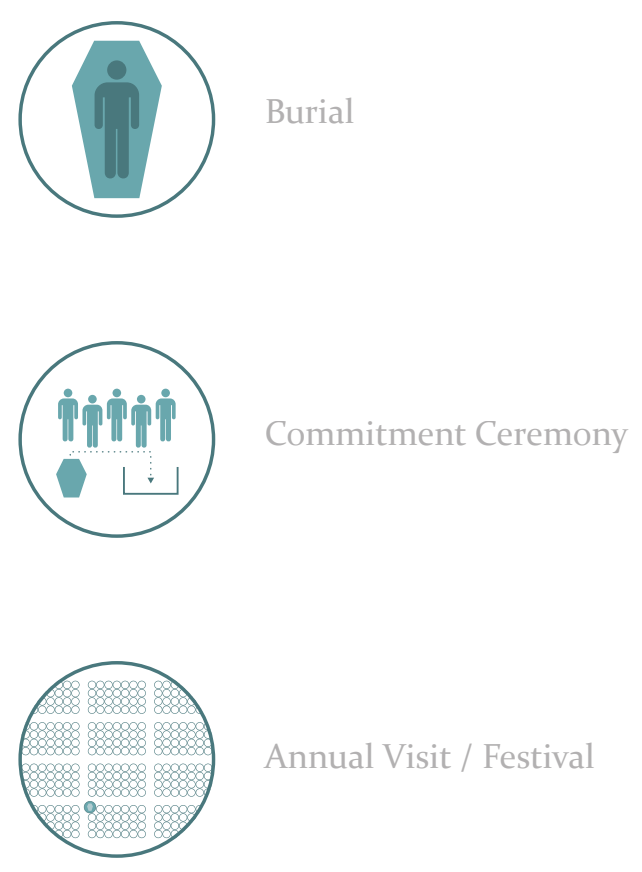

Annual Visit / Festival

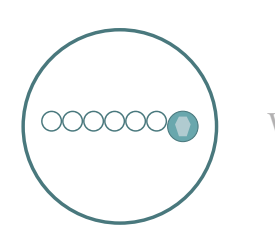

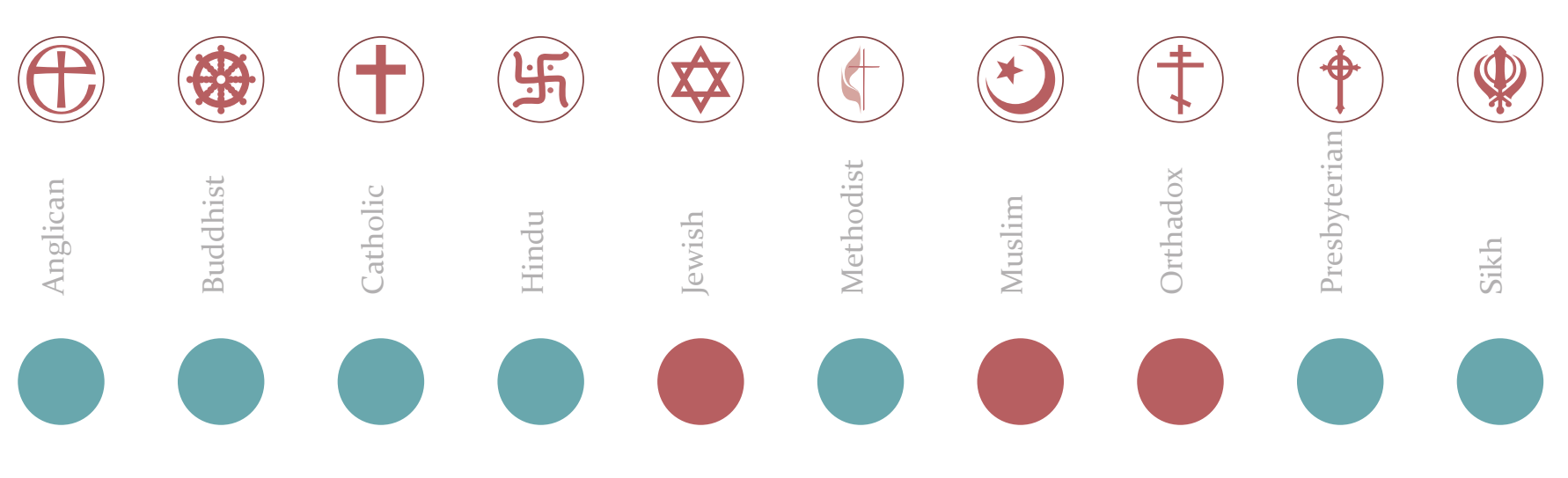

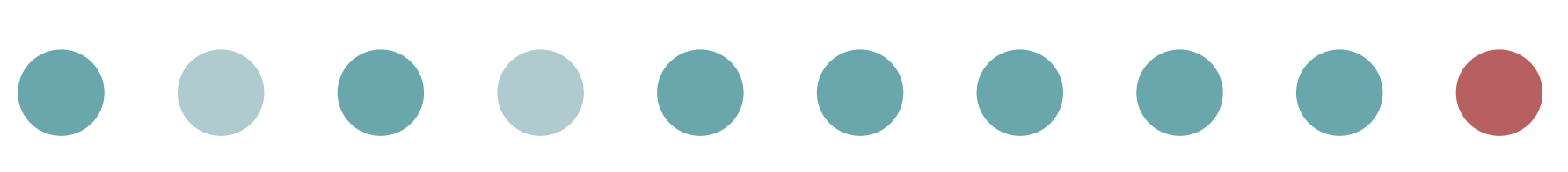

$O \bigcirc$
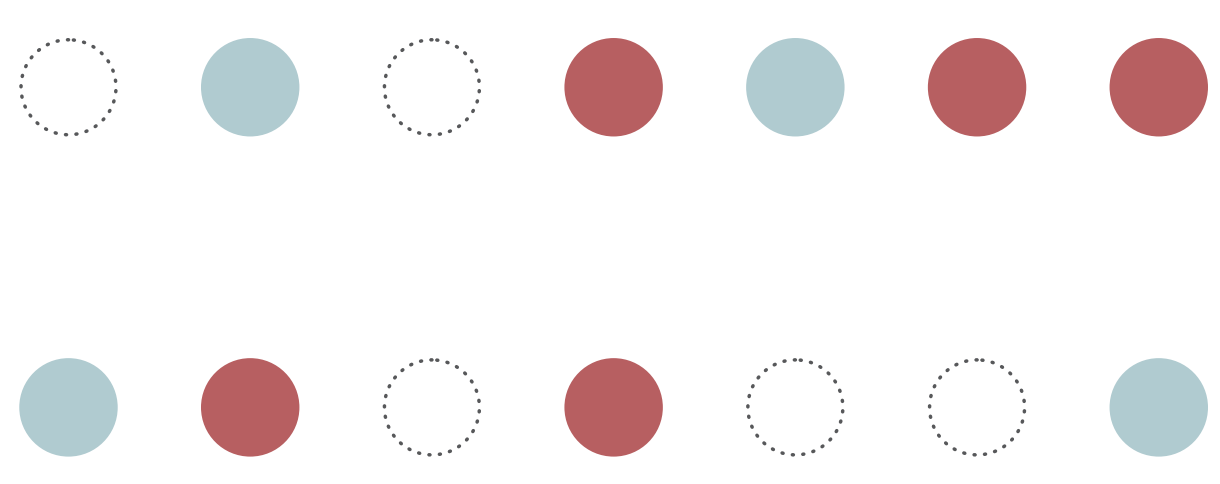

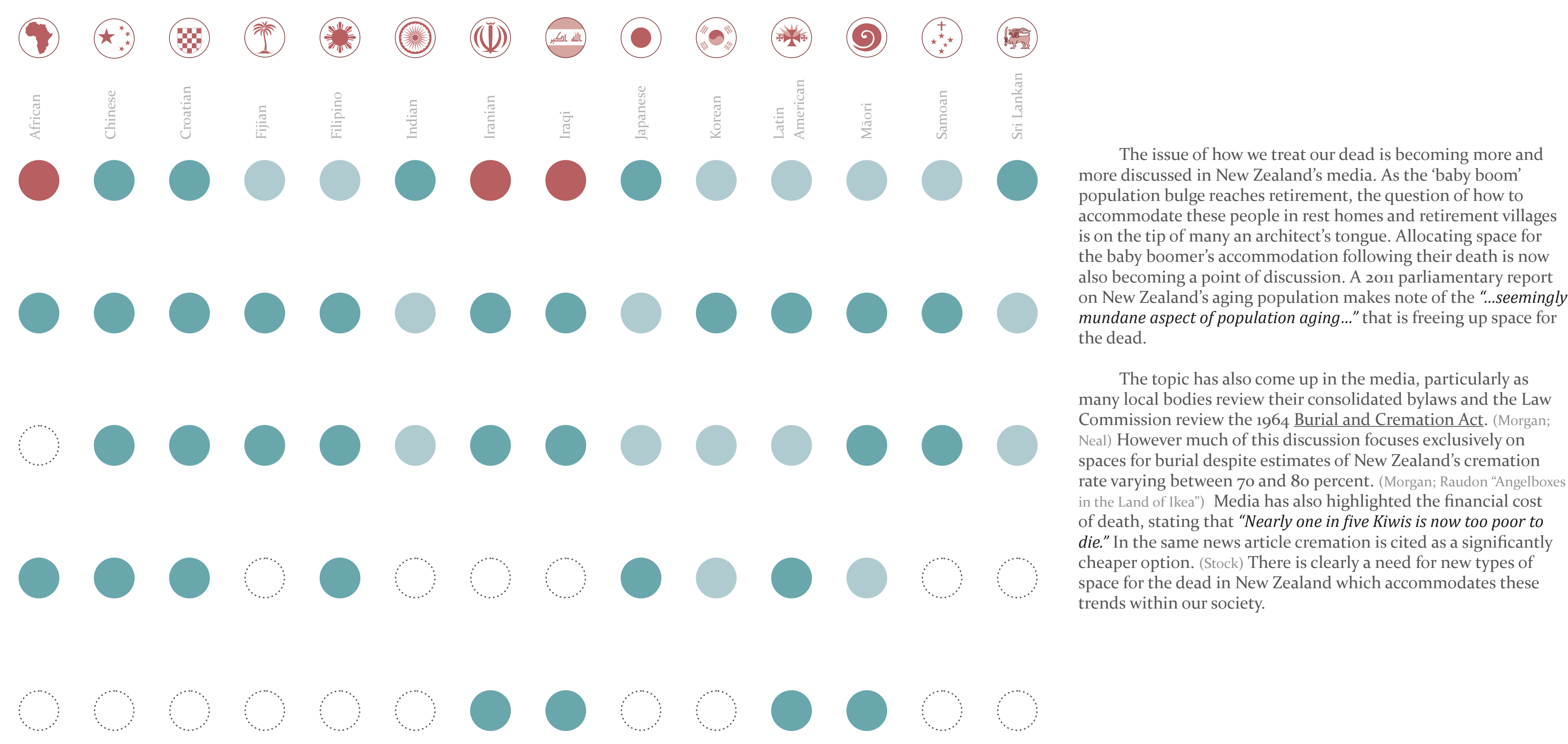


\section{Approaches}

The issue of how to find space for the dead is seldom approached by spatial designers. Worpole argues that: "Few, if any, modern urban planning models make reference to provision for burial or even memorial..." (31) and indeed, while several modern approaches to the design of cemeteries have arisen, there are few attempts to define other types of space for the dead. In particular there are very few attempts to find ways of inserting these land uses withi our settlements rather than at the edges where "...land values and eschatological values can be had at reduced cost." (31)

An exception to this is A Pattern Language by Christopher Alexander. The book, an urban planning model of sorts, describes in reasonable detail a scheme for a series of spaces for the dead.

In his 'pattern' number [70] - Grave Sites he decries the building of "massive cemeteries" (356) arguing that "...the presence of the dead among the living will be a daily fact in any society which encourages its people to live."(354) Instead he argues for a far less centralised system where land is allocated "...throughout the community as grave sitescorners of parks, sections of paths, gardens, beside gateways..."(356) This proposition certainly addresses the issues outlined in this thesis, and has served as an initial driver for the research.
However Alexander also proposes that "... a person can choose a spot for himself, in parks, common lands, on his land." (356) While he never elaborates beyond this rather ambiguous point, the suggestion seems to be that control of where spaces for the dead are placed would be given to the individual, rather than it being a collective decision. This seems to run in contradiction to his call to "...reinstate the connection between burial grounds and local communities." (365) as the spaces for the dead would no be collected for the community, but instead dispersed for the individual whim. However Alexander's general proposition, that of decentralising the cemetery, is an important one.

The 'pattern' number [66] - Holy ground, referred to in [70], describes his proposed techniques for spatial arrangements in places of spiritual significance. He argues that such places exist “...even if it is not associated with any particular religion." (334) $\mathrm{He}$ proposes to use a series of " nested precincts, each marked by a prateway, each more progressively private." (33, gateway, each more progressively private... (334) This gradient of privacy allows the space for the dead to be screened from the surrounding environment without having to push it out entirely.
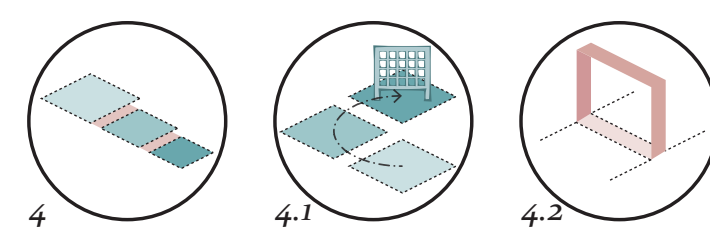
Gateways mark the transition between these spaces.

However, these are not necessarily conventional gates but instead conform to another of Alexander's patterns: number [53] - Main Gateways and [112] - Entrance Transition. In describing these gateways he suggests the use of sharp level transition (227) or surface quality $\left(55_{1}\right)$ to make clear the transition between one of these nested precincts and the next. His main insistence is that these gateways be physical things, rather than borders which exist only within the occupant's mind.

This represents one of the few attempts to outline a plan for the distribution and design of spaces for the dead.

Though not flawless, and still centred clearly around burial as a means of interment, it provides a valuable set of guidelines to begin exploration of new types of space for the dead in the contemporary city. 


\section{Conclusions}

The context surrounding space for the dead, while perhaps underaddressed in spatial design disciplines, is a field of literature too broad to be comprehensively covered in a design research thesis. However by focusing on issues of particular relevance to the proposition of this thesis, several points of use in the task of assembling design principles have presented themselves.

Historically, the distribution of space for the dead has been related to the population densities of the community it serves, with denser communities generally shifting their spaces for the dead to the periphery

However it has been argued by many that this shift to the periphery has negative effects. Whether there is truly a modern societal taboo surrounding death is much debated. But what is clear is that we have, at least, developed an aversion to the physical presence of death. $(1 \& 3.1)$

This is exemplified in the long standing debate of living versus dead memorials. The concept of integrating a utilitarian land use with the space of commemoration has proved to yield positive results (2.2). However some physical presence directly associated with commemoration is generally still necessary for the commemorative message to be clear.

This aversion to the physical presence of death can be seen in New Zealand as well. Communities here are often sensitive to the introduction of spaces associated with the dead into established neighbourhoods. Any attempts to infill these spaces must take this into account.

New Zealand does, however, have its own endemic culture of death: we have developed a preference for highly individualised ceremonies. This preference for individualisation must be catered to in new spaces for the dead. The speed at which this cultural shift has occurred also indicates that we are relatively receptive to changes to our practices around death. (5)

While few spatial designers have tackled the issue,

Alexander's A Pattern Language an interesting proposition for the decentralisation of the cemetery. His spatial schemes for a series of nested precincts with clear transitions provides a good mode for a privacy gradient in a space allocated for the dead. (4.1 \& 4.2)
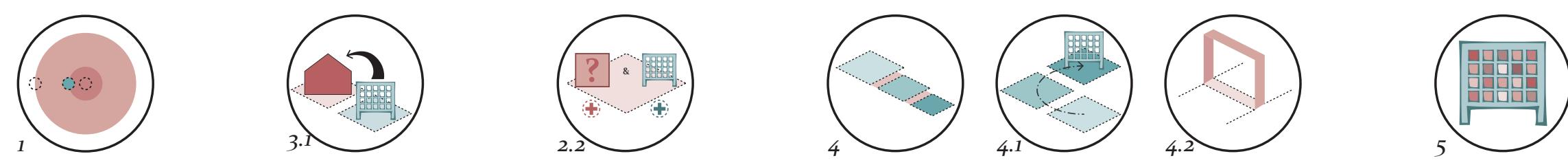


\section{Precedents}





\section{Introduction}

A s highlighted in the previous chapter, the Columbarium is by Ano means a new type of interment. However there have been relatively few attempts to actually 'design' these walls, instead they are often simple free standing walls with a grid of niches of arbitrary size, with little thought given to their placement or assembly.

This chapter seeks to review existing practice in the design of columbarium walls, with particular attention given to contemporary examples where some notable effort has been put in to the wall's design. These studies are intended to refine and add to the design principles, both in terms of site selection, and site design. This has been achieved through a graphical analysis of five different contemporary columbaria.

Several of the issues established in the context chapter have served as the initial drivers for these studies. Their location relative to community centres and their integration with other programmes on site has been identified. While the majority of them are built within existing cemeteries, there are some which are separate entities. Even some of those based within cemeteries serve additional spatial functions such as resting areas.
At a more detailed scale the level to which the physical presence of death is visible has been identified. To what extent the niches for interment can be 'individualised' has also been reviewed. Weather there is adequate space provided for an interment ceremony is investigated. Finally a spatial analysis based on Alexander's 'patterns' of nested precincts and gateways has also been performed so that the pattern can be refined towards the specific form of the columbarium.

Through these analyses the design principles are expanded and refined, particularly at the detailed scale, in order to make use of them in the design case study. 


\section{De Nieuwe Ooster Columbarium}

Sitting at the former periphery of Amsterdam City, the De Nieuwe Ooster Cemetery has now been surrounded by medium density housing developments and absorbed by one of many new suburbs. In response to this architects Karres En Brands have carried out a redevelopment of a section of the cemetery, including a Columbarium with space for 1,0oo urns. (Karres En Brands)

The modern sculptural nature of the columbarium contrasts strongly with the surrounding traditional cemetery. However the cemetery's opening hours mean that an inward facing series of intimate rooms can be created without crime prevention worries which might be present in a less controlled environment.

Columbarium Plan Scale - 1:250
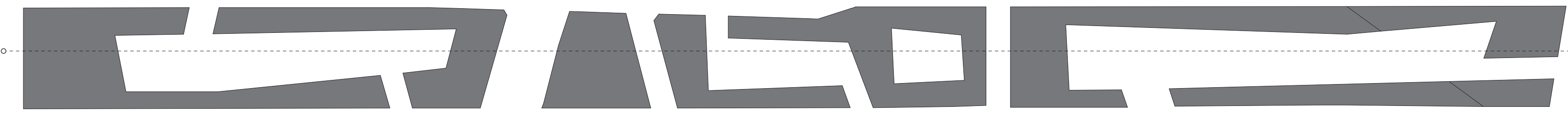

Columbarium Section

Scale - 1:250

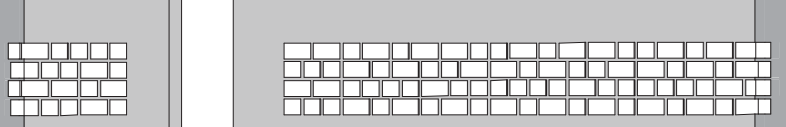
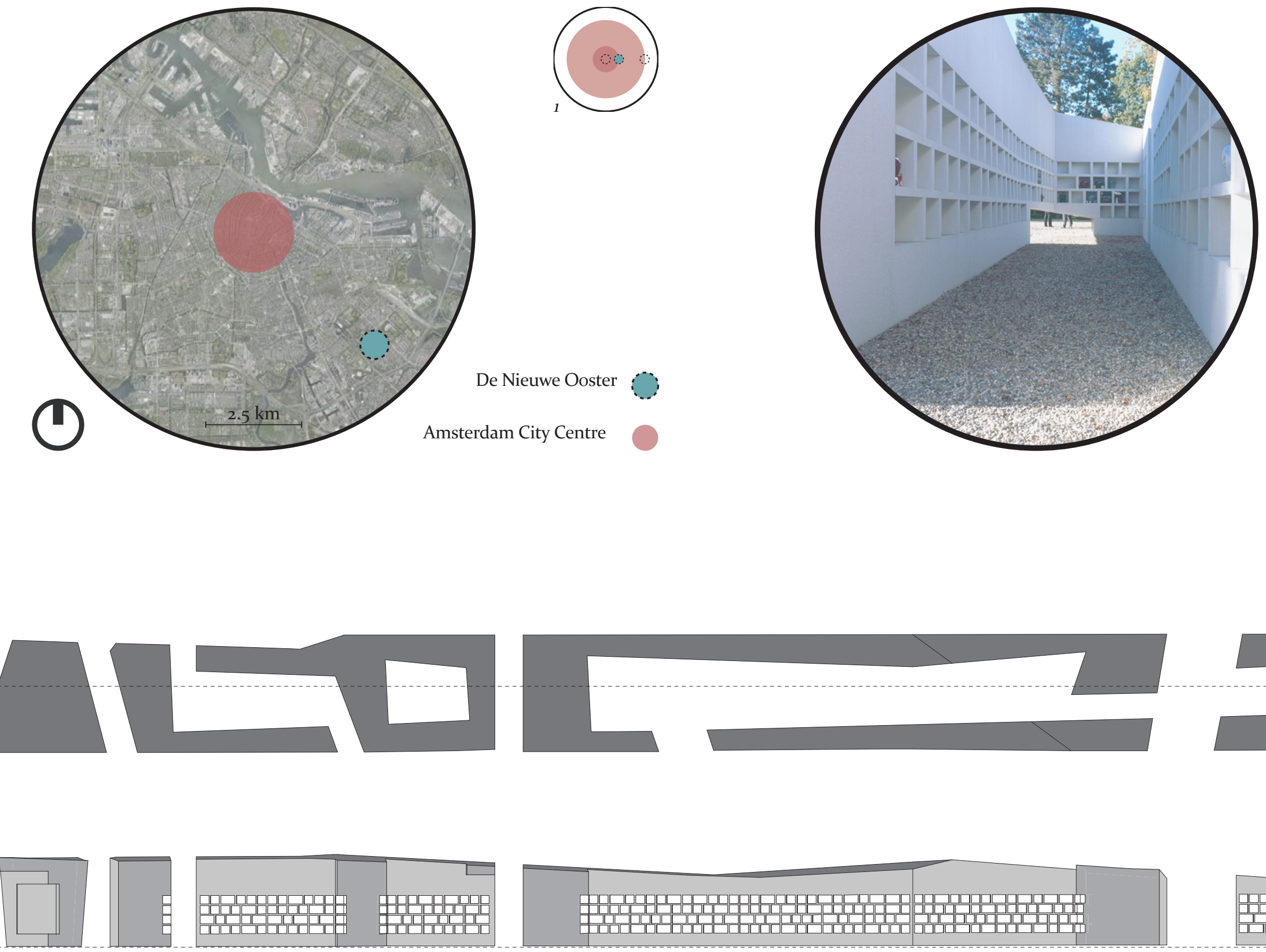

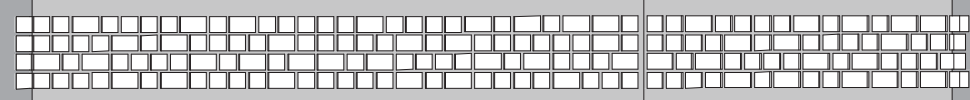


The transitions are clearly marked by gaps in the wall itself.

However there is nothing in these 'gateways' to notate weather

they move you through a commemorative space or a thoroughfare.

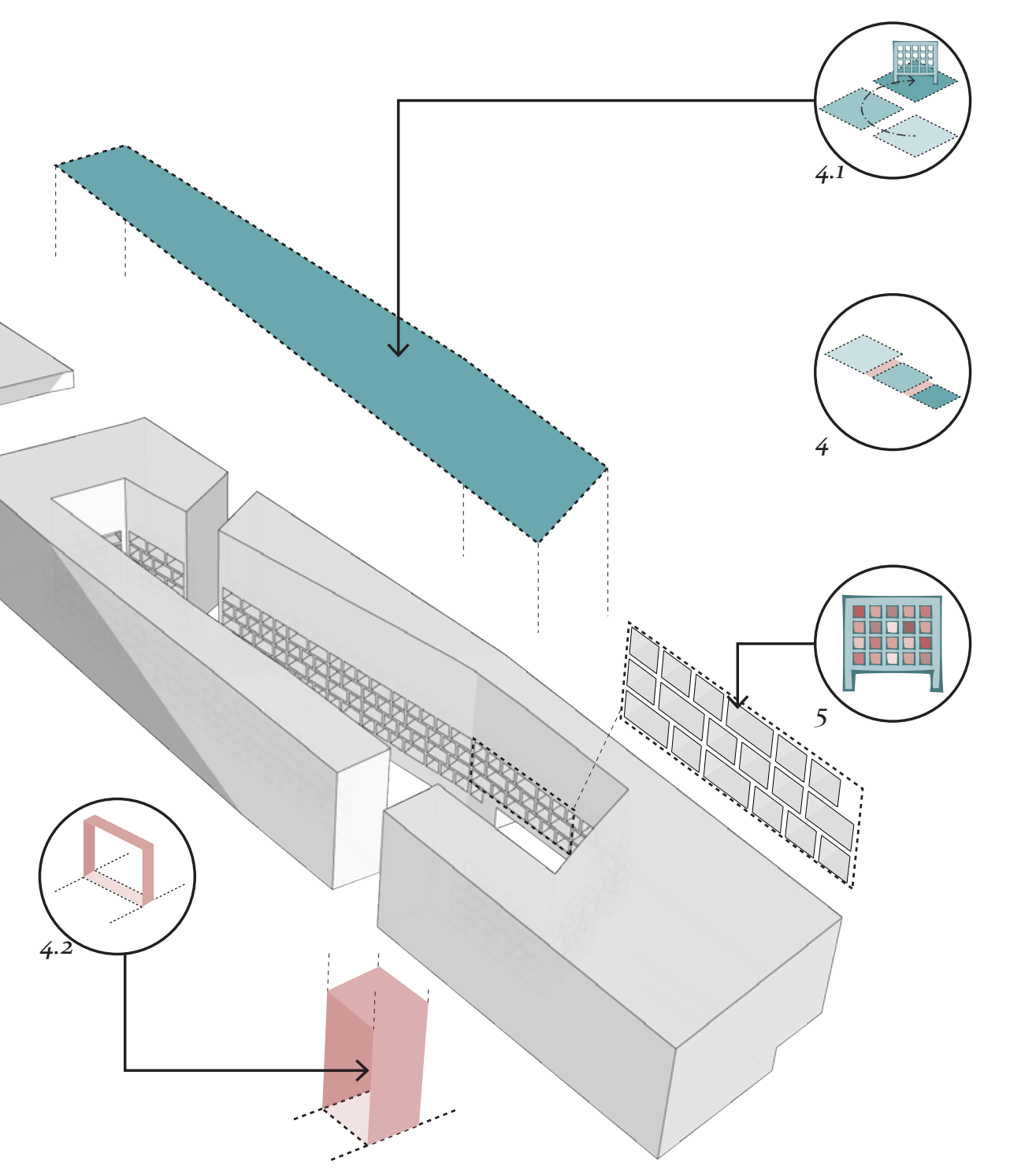

The inward facing niches of the columbarium provide little space for commemorative ceremonies. This means that these ceremonies are in-fact abstracted from the act of interment.

The commemorative spaces, while small, are reasonably distinctive. However there is little attempt to create a layered intimacy, although this is perhaps due to the surrounding spaces being part of a larger cemetery, with an existing sense of intimacy.

In a small step towards individualised interment, the provision of several different niche sizes represents a level of potential customisation for users. Space for flowers and other offerings is also provided. 


\section{Friedhof Hörnli Columbarium}

This is another columbarium retrofitted to an existing cemetery in Basel, Switzerland. The Hörnli cemetery has progressively encroached on the woodland reserve which it borders as demands for land have increased. The columbarium, designed by Vetschpartner and completed in 2003, has been added to the eastern edge, close to the surrounding residential land use.

The columbarium's honeycomb structure has been orientated to catch the light, particularly when more niches have been filled and their coloured glass 'caps' create a mosaic pattern on the commemorative space.

Columbarium Plan

$$
\text { Scale - 1:250 }
$$
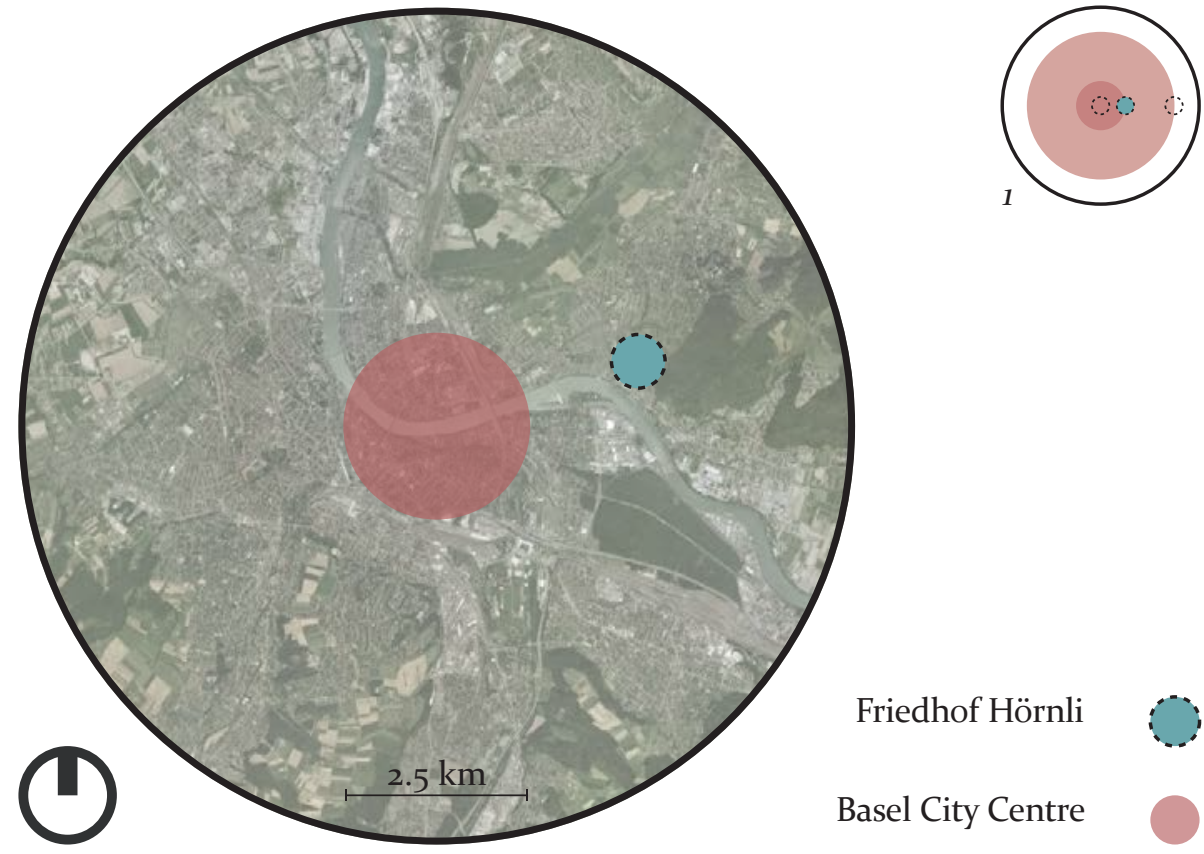

Friedhof Hörn Basel City Centre
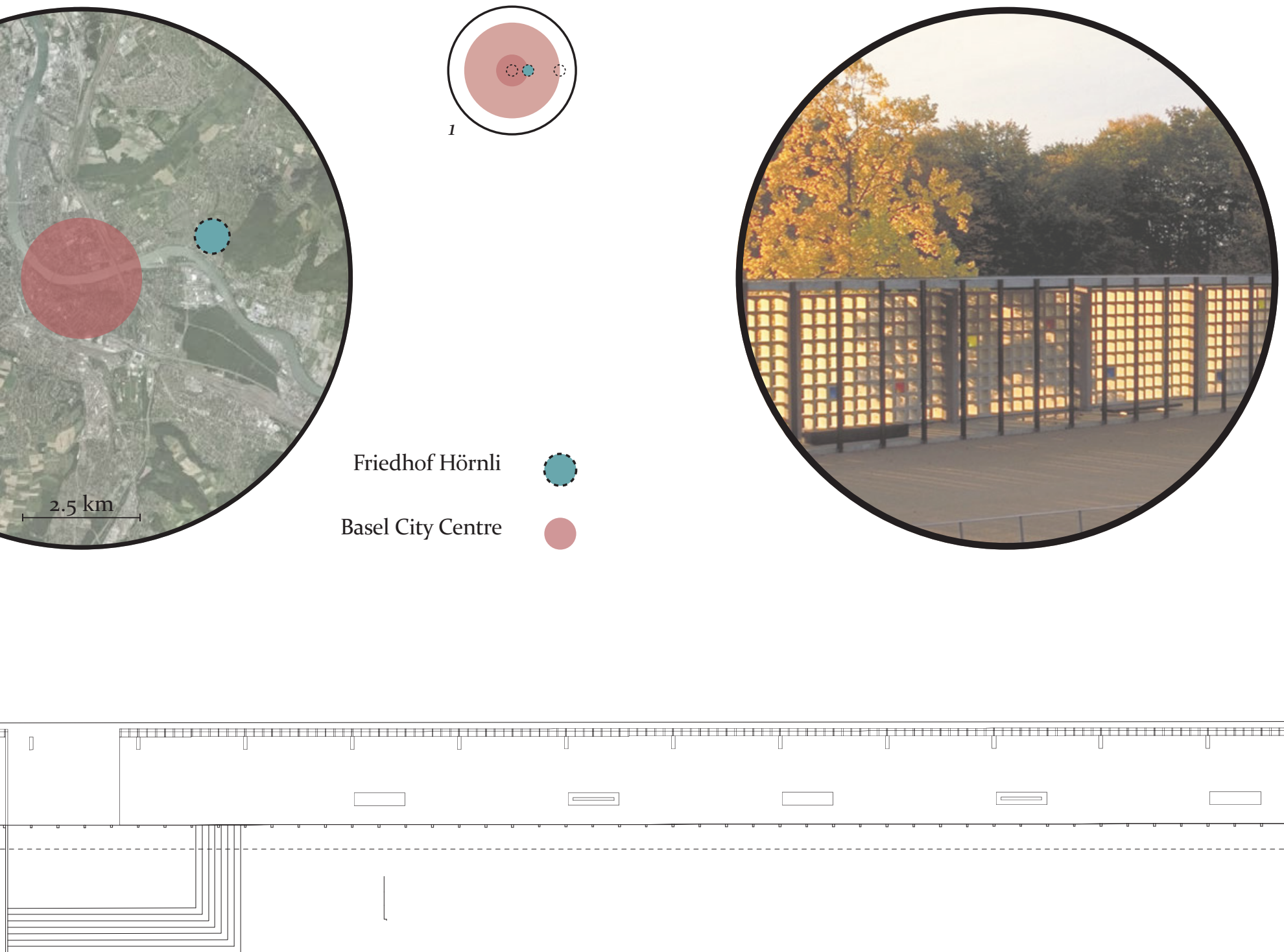

Columbarium Section

Scale - 1:250

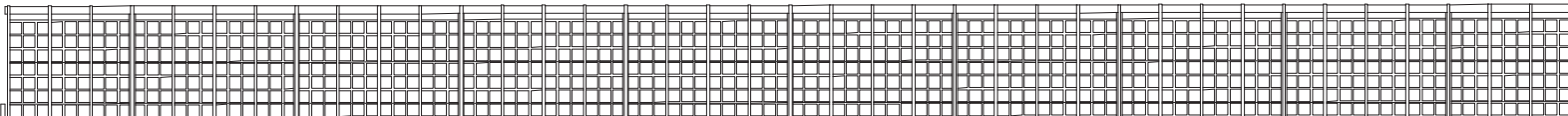




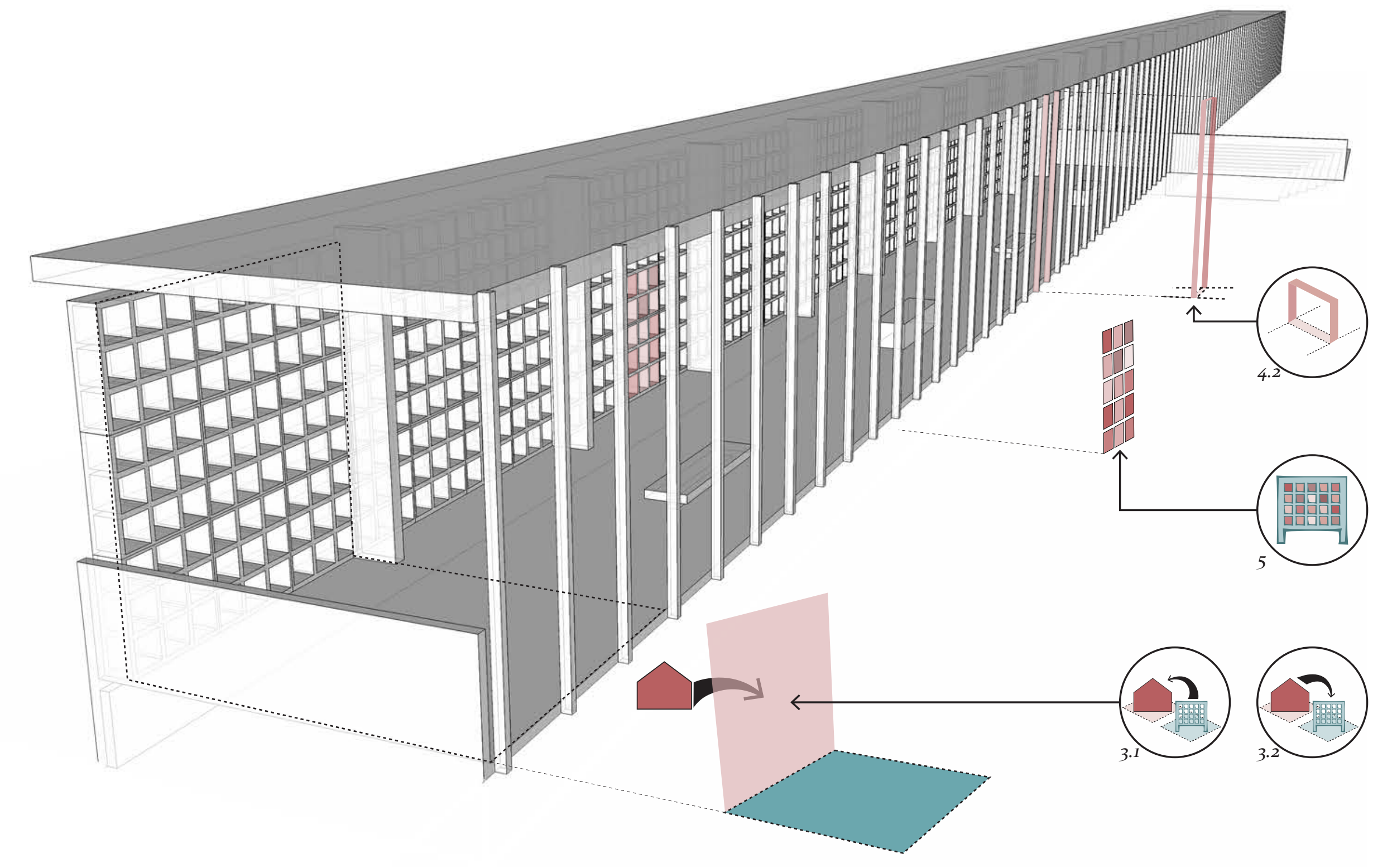

The structure supporting the columbarium's canopy marks the transition in to the commemorative space. However as this transition only occurs once, it does little to create a sense of layered intimacy.

The multicoloured glass 'caps' to the columbarium's niches provide a good example of a potential for individualisation which actually improves the effectiveness of the overall design, by creating a mosaic like effect in the honeycomb structure.

The surrounding land uses are screened from the commemorative space by the columbarium itself. It is not completely opaque (the niches have a frosted surface on the far side) but provides a level of ambiguity to the purpose of the structure. 


\section{Friedhof Steckborn Columbarium}

Although also situated in Switzerland, in the small municipality of Steckborn, this columbarium occupies an entirely different context. It is attached to the small churchyard near the town's historical centre.

Vogt Landscape Architecture engaged in the project by first carefully considering the nature of the town centre and its relationship to the churchyard.

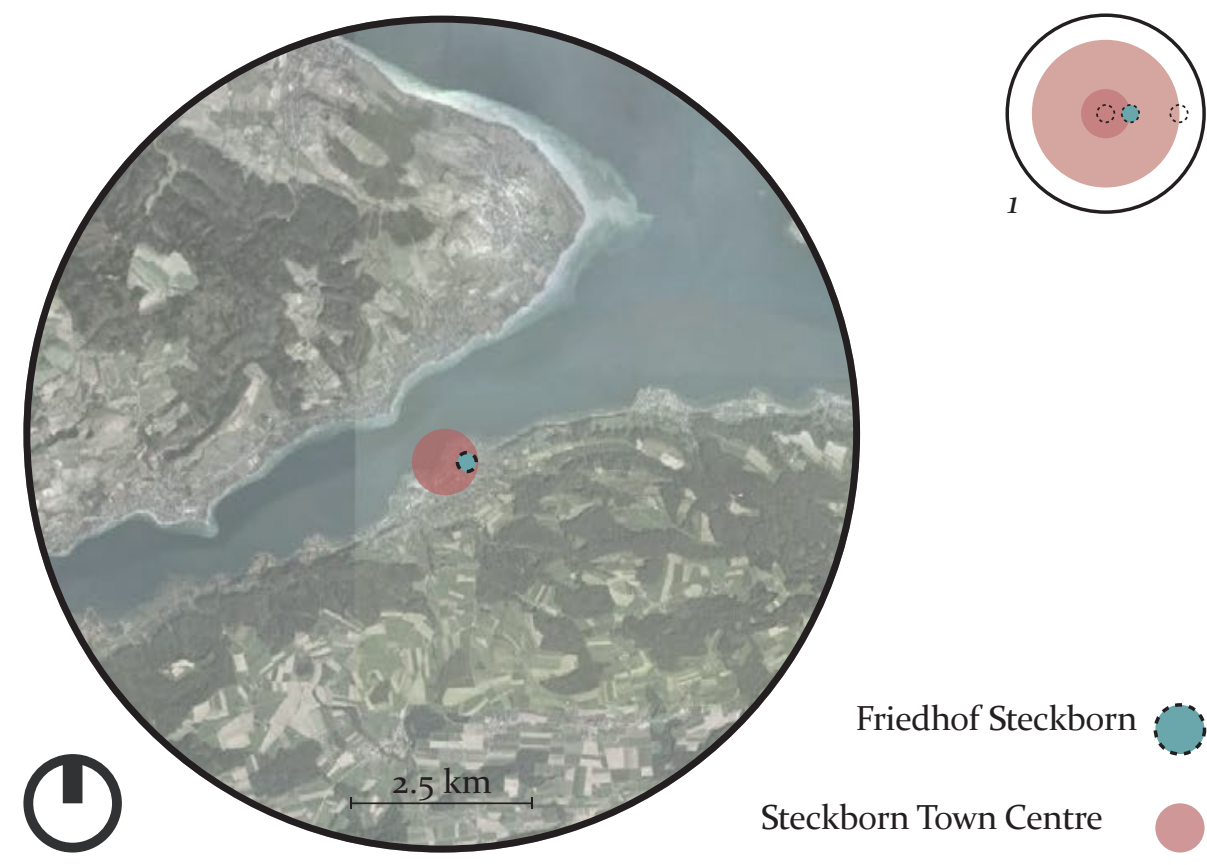

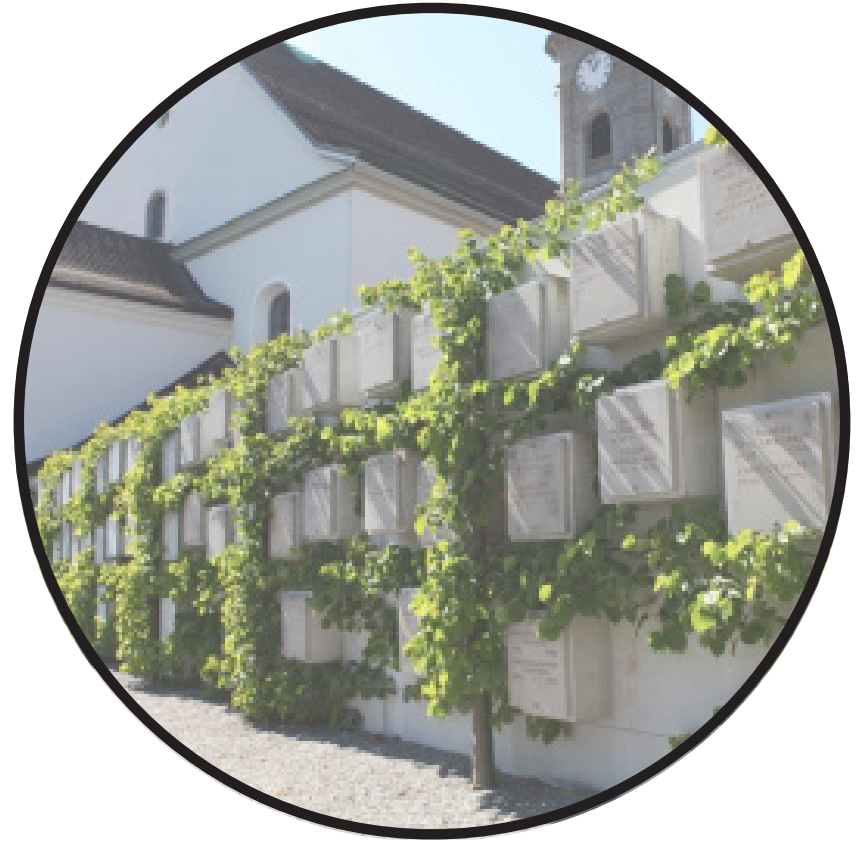

Columbarium Plan

Scale - 1:100

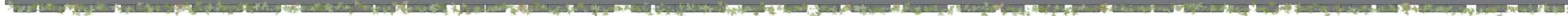

Columbarium Elevation

Scale - 1:100

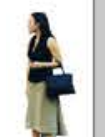

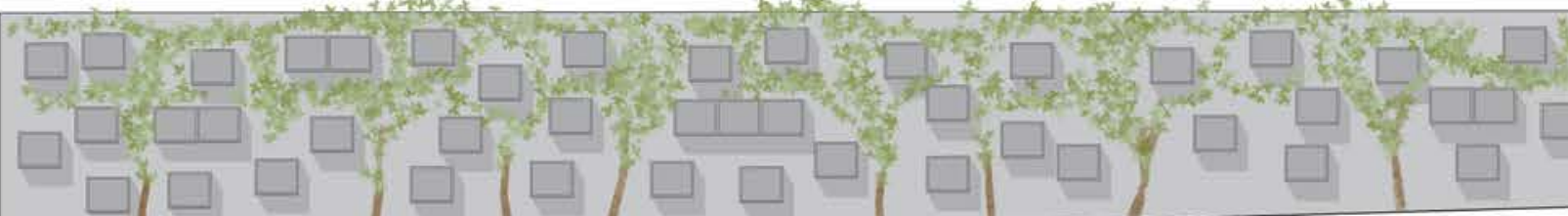

$7 \square, \square \square=(\square(\square) \square \square \square(\square) \square(\square)$ 


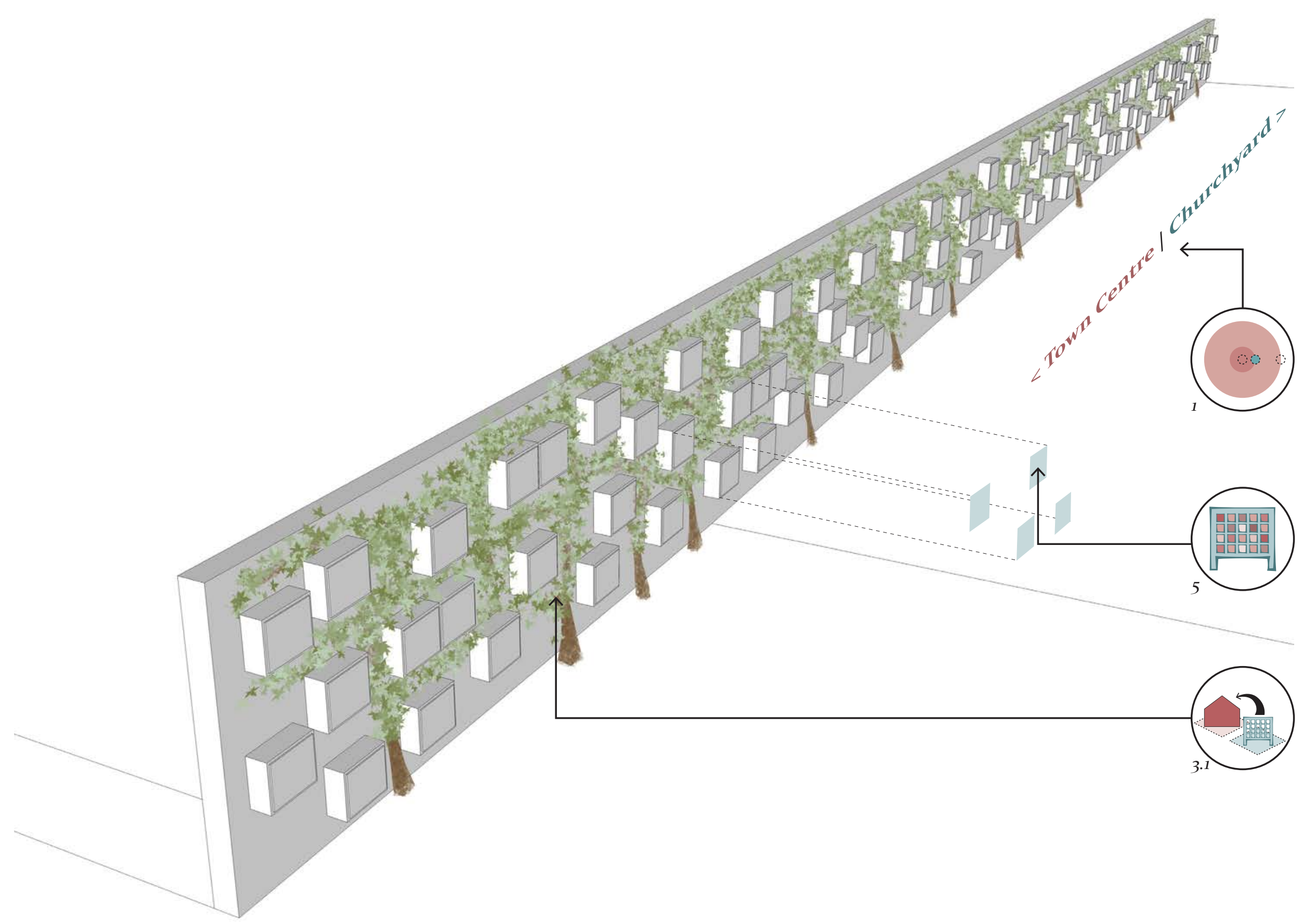

Situated at the edge of the town square next to a church, the columbarium serves as a transition between the square and the churchyard. While not central to the town it still holds an important presence.

The engraved stone 'caps' of the niches lack potential for individualization.

The trained trees serve to limit the prominence of the niches, meaning that effects on the surrounding land uses are limited. 


\section{Westminster Urban Columbarium}

Located at the edge of the CBD in the sprawling mid-west city of Minneapolis, Minnesota, the Westminster Church is perhaps the most urban site amongst these projects.

Cohen + Partners have established the columbarium and associated courtyard as elements related to both the church and the wider city through the key element of the copper screen running along the public / private northern border of the site.
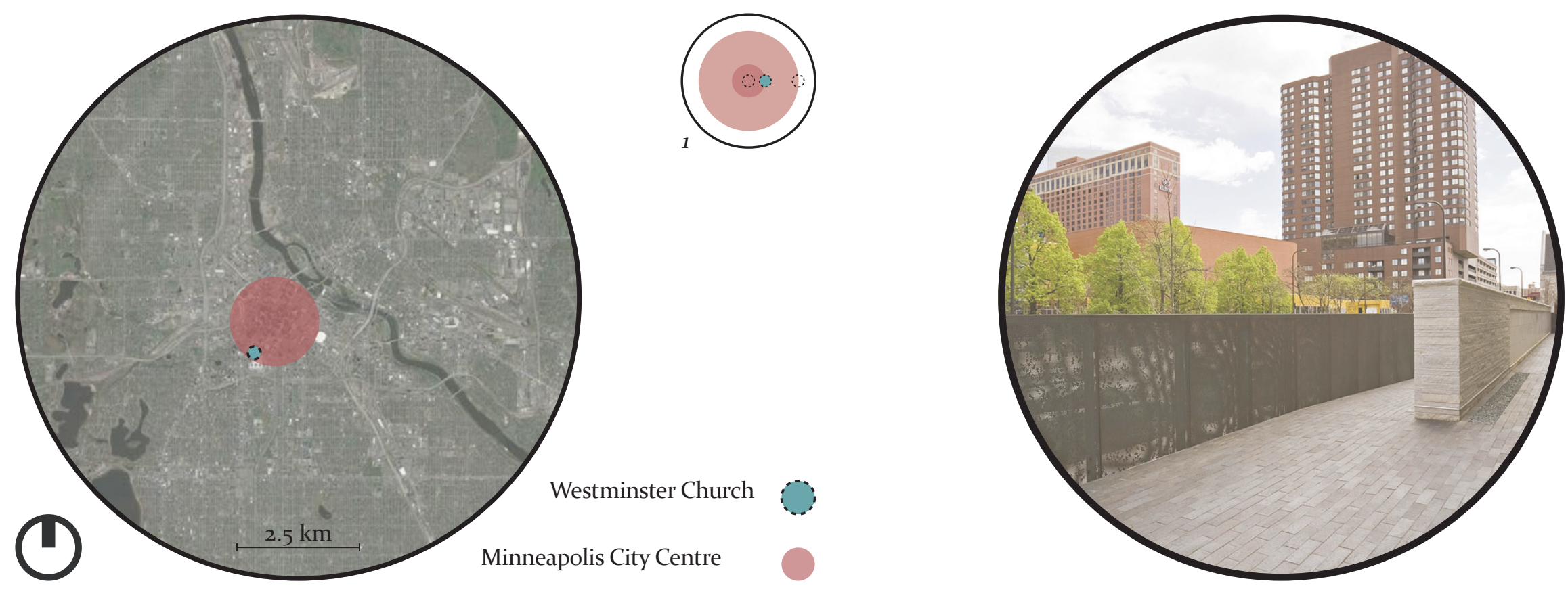

Columbarium Plan

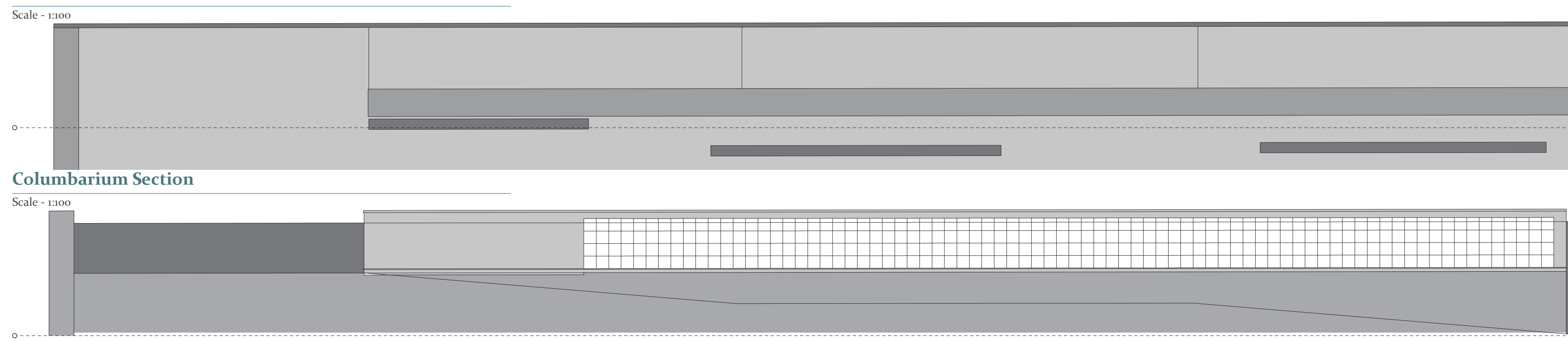




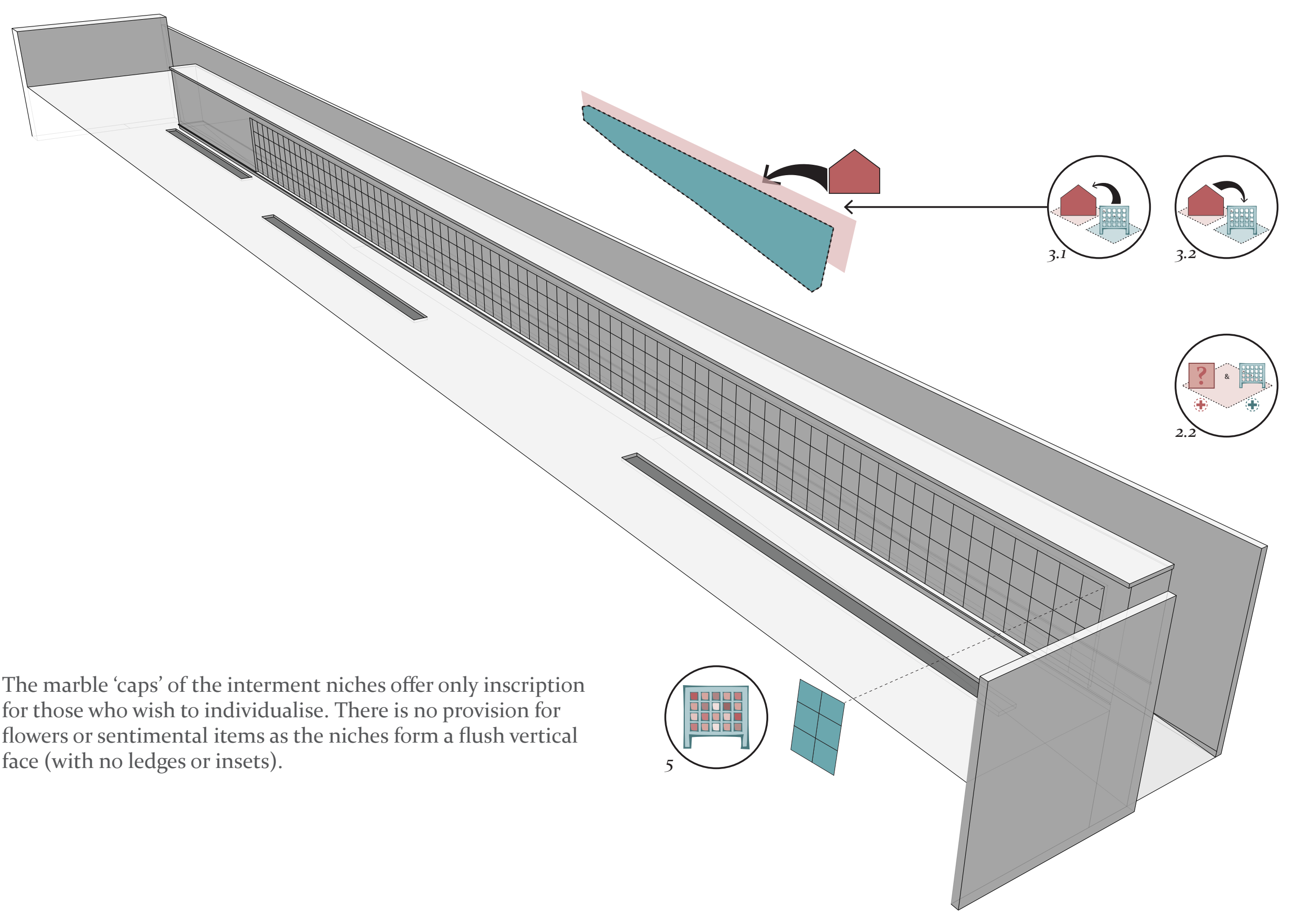

The perforated steel screen which sits at the edge between the courtyard filters views from the surrounding land uses to the columbarium, mitigating the impacts on and from near by land uses. At the same time it is not entirely opaque .

Rather than simply being an extension to an existing cemetery , the columbarium is combined with another land use. A memoria courtyard is included within the scheme, adding further value to its installation via public space. 


\section{Arradon Columbarium}

The expansion of the cemetery of Arradon (a medium sized town in Brittany, France) in to a neighbouring parcel led to the inclusion of a columbarium and associated spaces.

Urban designer Philippe Madec placed an emphasis on an intimate space for the columbarium, while leaving the surrounding gardens and burial spaces more open.
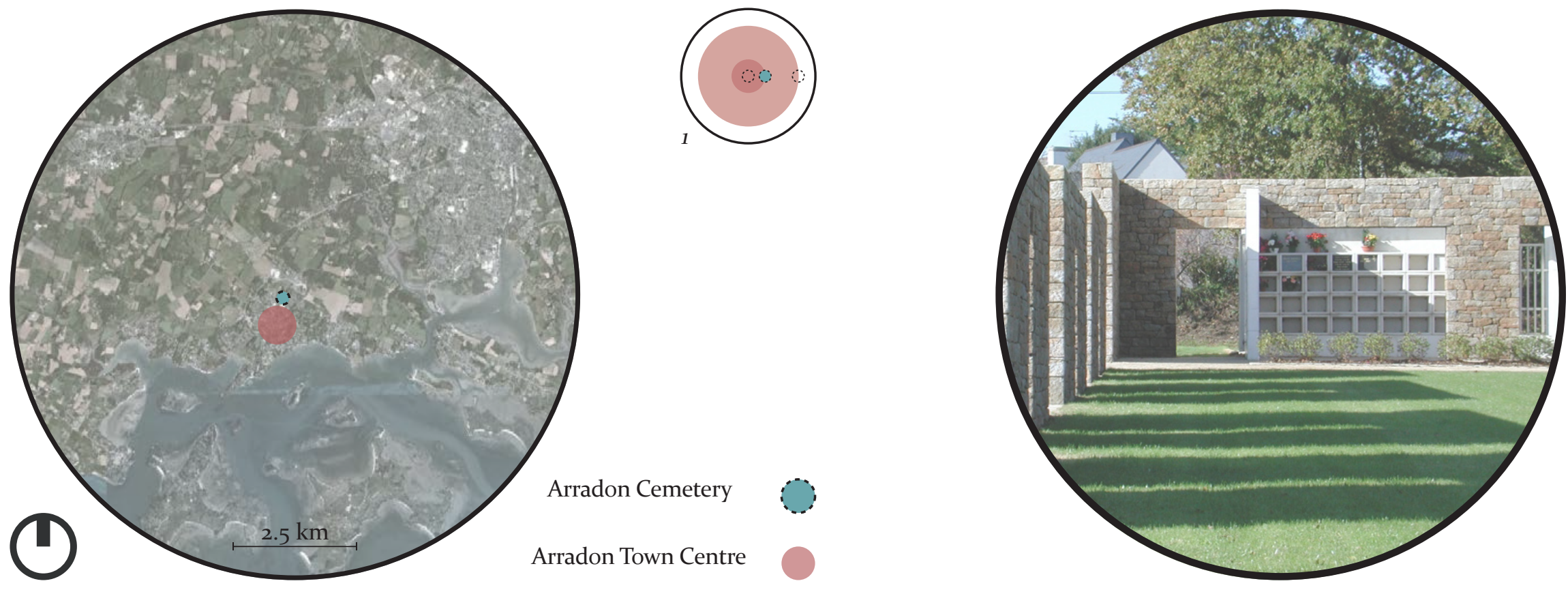

Columbarium Elevation

Scale - 1:50

Columbarium Plan Scale - 1:200

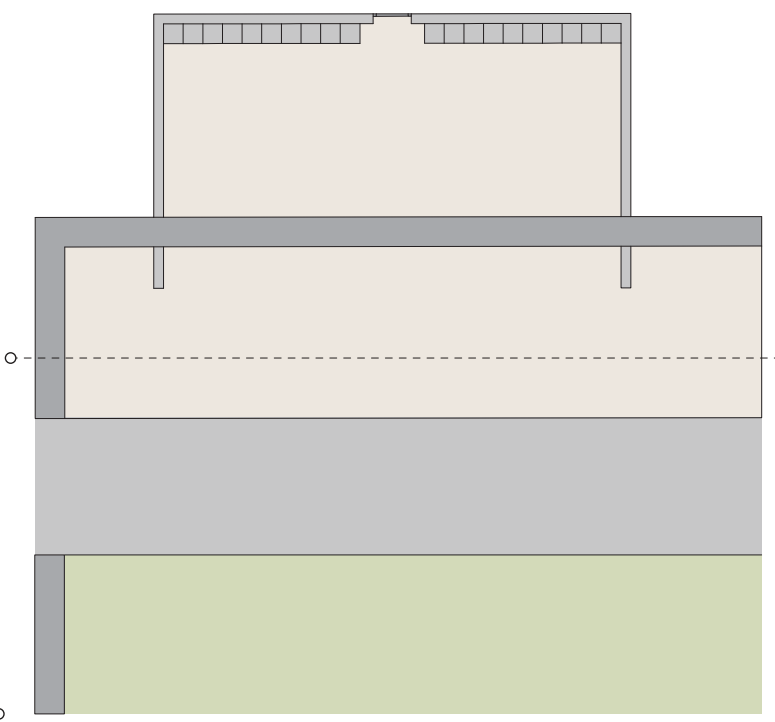




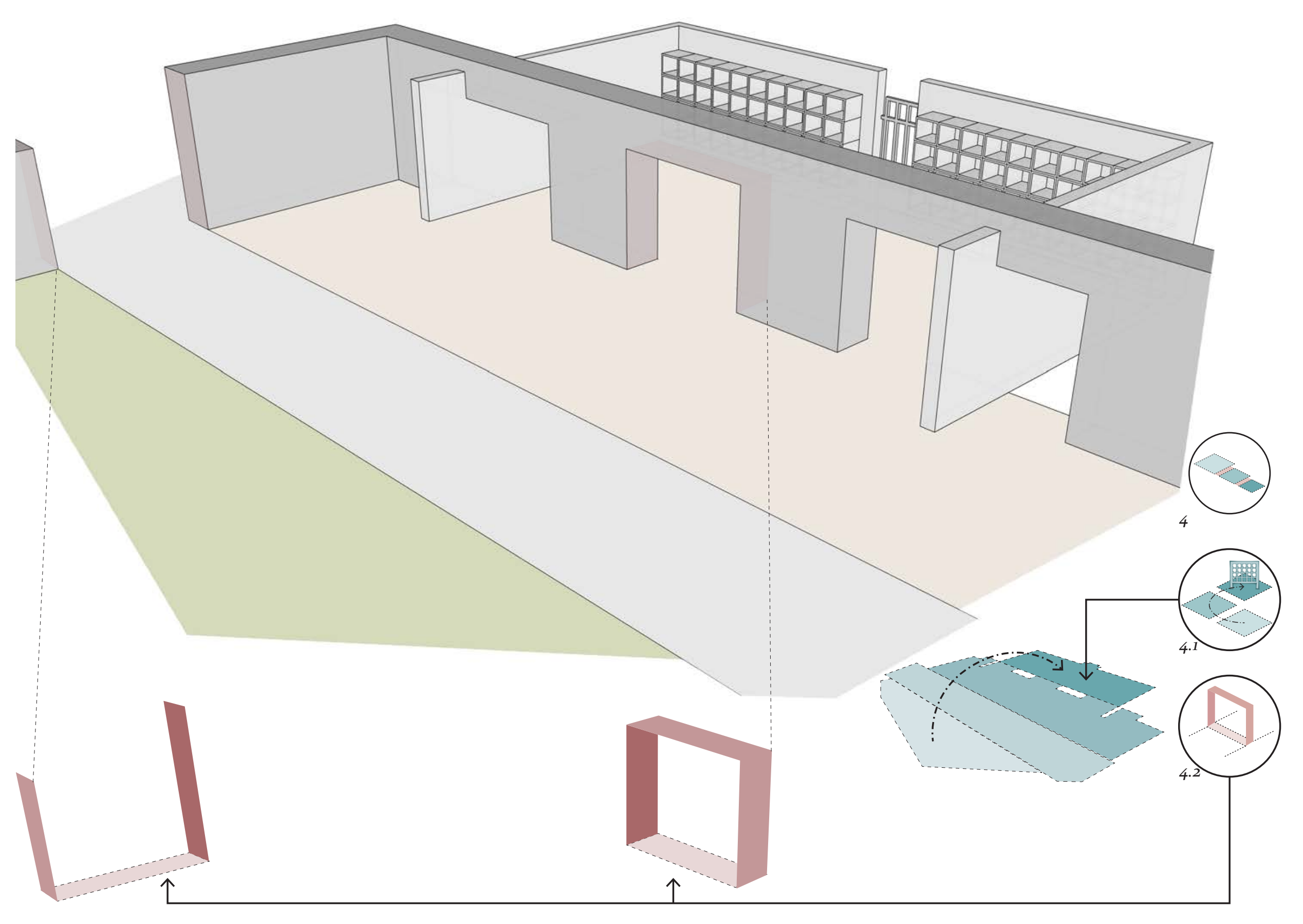

The columbarium is the best example of a layered approach similar to that proposed by Alexander.

A series of distinctive spaces lead in to the commemorative space, where the columbarium is placed. Surface materials and progressively more enclosed spaces create this distinctive progression.

Clear transitions between these spaces are expressed through portals and gateways. 


\section{Conclusions}

Despite the fact that very few columbaria are actually 'designed' spatial designers have on occasion attempted to move away from the status quo of columbarium design. The projects outlined here serve as examples of existing approaches to several of the issues outlined in the previous chapter.

The location of many of these columbaria is within the arge cemeteries at the periphery. Friedhof Hörnli serves as an example of this, sandwiched between the low density suburbs of Basel and a woodland reserve which it is slowly eating away at. However Friedhof Steckborn and the Westminster Columbarium are exceptions. Steckborn's columbarium is a transitional public space between the town centre and the small churchyard. Similarly Westminster's columbarium is attached to the inner city Church in a similar fashion to a churchyard, located at the community's centre, not the periphery. (1)

Westminster's columbarium is also integrated with a public courtyard, a utilitarian land use which gives the project obvious value to the surrounding community while still introducing a plainly commemorative element, and the physical presence of death. (2.2)
The inhabitants of nearby land parcels may have been sensitive to the introduction of a columbarium so close to the centre city. However by screening the space from the sidewalk with a perforated copper panel the existence of the columbarium, while not necessarily 'hidden' was made a more subtle fact. This also creates a level of privacy and intimacy within the space, something which is likely to be appreciated by those visiting loved ones. A similar effect can also be seen at Hörnli where the Columbarium itself serves as a partial screen between the newer and older sections of the cemetery. (3.1 \& 3.2 )

While the principle of 'Nested Precincts' is not present in most of the projects, with clear transitions and distinctive spaces playing only a minor role in spatial organisation, the Columbarium at Arradon Cemetery in France provides a good example of similar spatial principles. Clear portals physically mark transitions from one space to another, and the spaces approaching the columbarium have distinctive surfaces. (4.1 \& 4.2$)$

These spaces leading up to the wall also provide ample space for commitment ceremonies. This can also be seen at Hörnli where a large limechip open space borders the more enclosed space of the wall. This can be contrasted with the inward facing De Nieuwe

Ooster, which provides very little space for commitment ceremonies. $(41)$
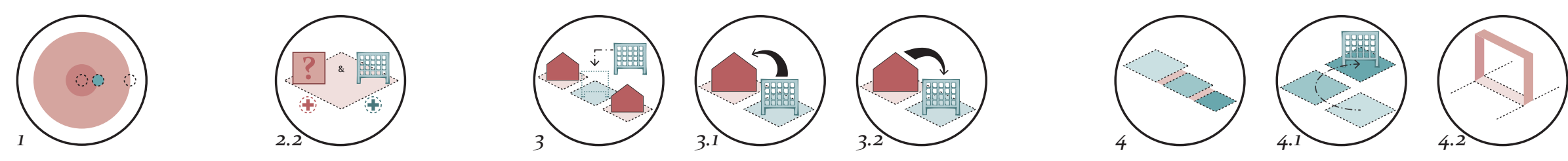
The Nieuwe Ooster columbarium does, however, provide some potential for individualisation. Niches of varying sizes are available and the niches provide space for flowers, trinkets and larger than normal urns. However a slightly more eloquent solution to a need for individualisation is seen at Hörnli where tinted glass panels of varying colours are placed at the front of niches, and

flower holders can be placed to the side of the niche. This is a strong point of difference with the more traditional etched marble faces of Steckborn and Westminster. (5)

These approaches to the issues around finding space for the dead constitute a valuable input for the design principles. They

provide a more practical, tangible side of the necessary background to the design research which will follow. 



\section{Design Principles}





\section{Introduction}

The thesis needs to contribute not only as a specific scheme for

1 Wellington city but as a more abstracted model applicable in a variety of different suburban environments.

This chapter is included not only as a lead up to the following proposition chapters, but in order to provide such a model. The design principles, built up through background research and initial design investigations, are the five main points which will be used in the design of a columbarium network across the city. The principles themselves, and their underlying logic, are described in this chapter. This is done without looking specifically at how they will be applied to Wellington city. As such they can be seen both as an output of the thesis in their own right, and a methodology which will lead in to the two design chapters which follow.

Each principle is described with its title, a symbol and a short single sentence description. This description is then elaborated on, with the underlying logic of the principle presented followed by an explanation how it might be implemented during design. 


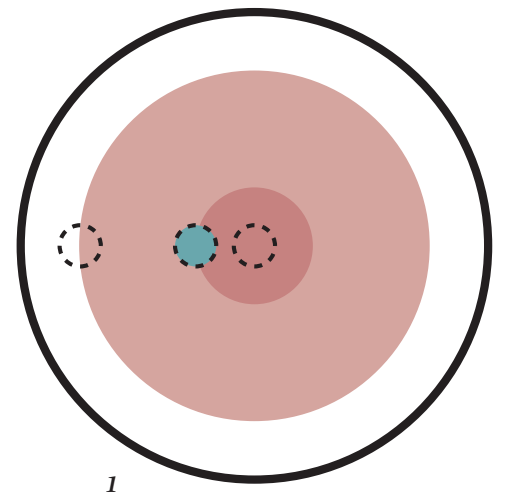

Informed Introduction

Aligning space for the dead with the community's core without undermining its existing significance to the community.

The negative implications of spaces for the dead placed at the city's periphery has been the subject of much discussion, including that of the background chapter, and is effectively the 'problem' to be addressed within this thesis. However simply returning the previous system characterised by the churchyard, where the space for the dead is tied closely to the community centre, is hardly the most viable option.

While these previous spaces developed in an almost ad hoc way amongst significantly lower populations densities, the forced insertion of such spaces in to the hearts of existing suburban communities would be unlikely to meet approval. With the 'taboo' surrounding death in contemporary society, community approval will always be difficult to win. However if focused on spaces in the absolute centre of the community, the likelihood for approval becomes almost nil.

However this does not mean that a new columbarium cannot align itself with the community's core; the area where the majority of community activity occurs. This is not necessarily the community 'centre' in the same sense that the church, town hall or post office

often are, but is rather somewhere where one could reasonably expect to see most residents of a suburb at some point during a given week.

It is important to have some idea of where this core actually is. Otherwise there would be the possibility of selecting a site that, while otherwise entirely suitable, is too far from this space to be experienced by the majority of inhabitants, defeating the purpose of a shift to the suburbs in the first place. Establishing where a community's core is requires a layered analysis, taking into account land use types, topography, circulation and built density. (Fig. 25) This core provides a constraint on the area in which sites can be pursued, helping to moderate the presence of death. 
Development Density

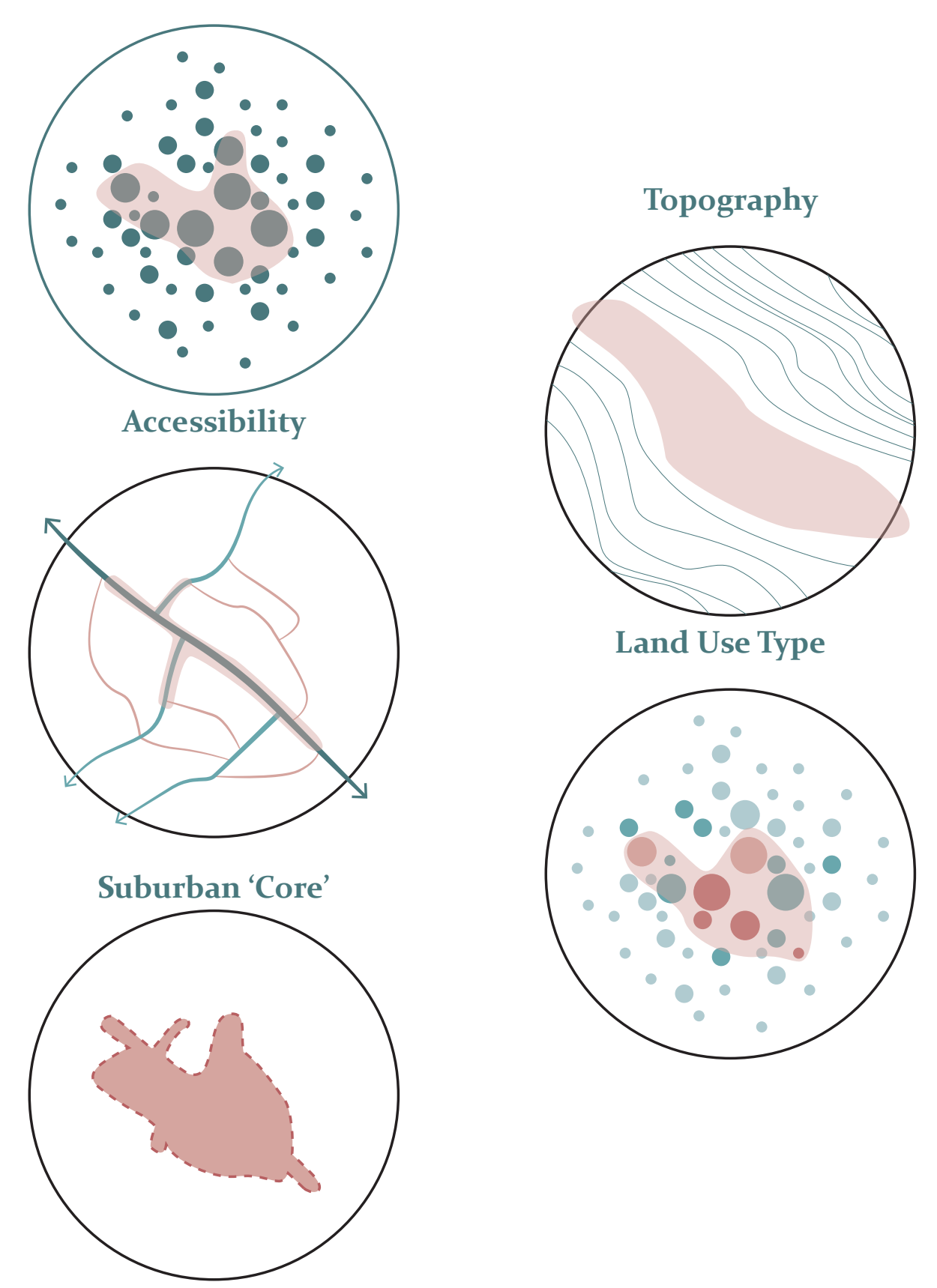

Figure 25. Core Identification Example Source: The Author 


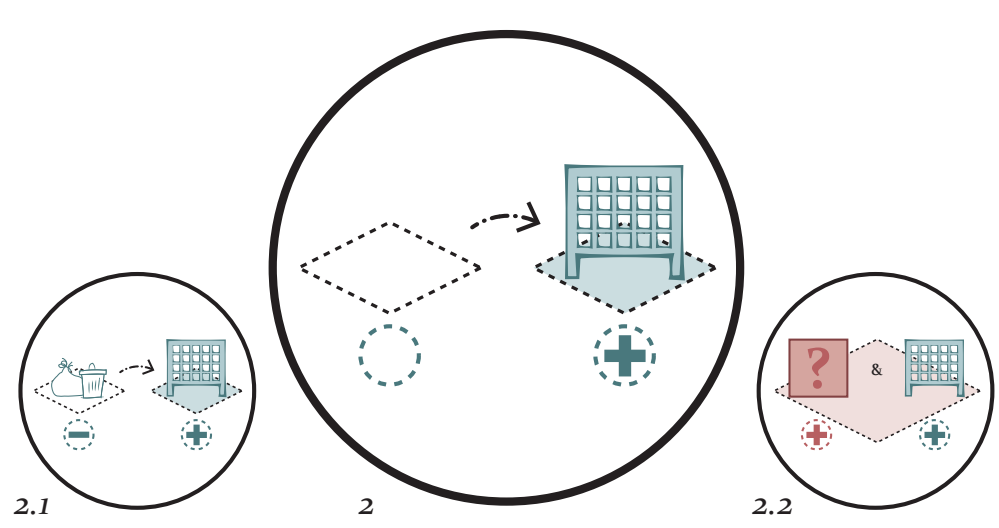

Obvious Improvement

Selecting underutilised sites where a designed intervention will be an obvious improvement. (2.1)

Integrate other land uses which are of clear benefit to the community with the space for the dead. (2.2)

In order for the community to actually accept infill of a columbarium, it is important that the intervention is a noticeably positive change for the community's environment. This is achieved first by choosing sites which currently have a generally negative impact on the suburb's ambience. Secondly it is achieved by

integrating some other land use of a more tangible usefulness to the community with the columbarium.

This section first describes a series of common types of underutilised spaces and reviews their suitability for the introduction of a columbarium. Next a set of land uses which could be integrated with columbaria are reviewed in terms of the potential interaction with a columbarium and their benefit to the community. 


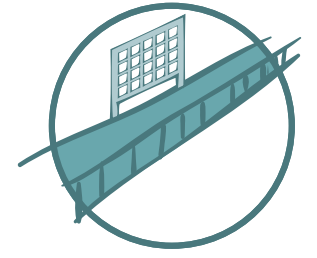

Public Pathways

Public pathways which connect different streets when a proper road is not possible often receive little maintenance or design attention, leading to underutilised spaces.

+ Longer Paths have room for further expansion if necessary.

- Constant thoroughfare means less privacy / intimacy.

- Linearity means less opportunity for design diversity.

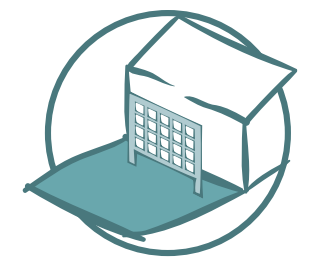

Building Edges

In certain circumstances the facades of buildings can be ignored or neglected by building owner, particularly when they are the 'back' end.

(1) Eliminates the need to design the back side of the columbarium.

- Also eliminates the possibility of access to both sides.

$\rightarrow$ Issues around privacy/ownership for both the building and the columbarium.

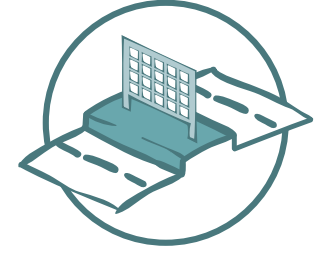

Road Margins

While roads often receive a disproportionate amount of maintenance, many suburban road margins and medians are often left to their own devices, barely utilised.

- The road acts as a buffer between the columbarium and other land uses.

$\rightarrow$ The issues surrounding road noises are increased with the inevitable proximity, and roads with margins are often busiest.

- Issues with pedestrian access are also likely, due to traffic

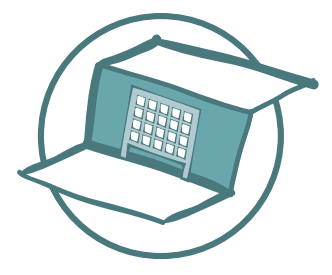

Retaining Walls

In many cities the retaining wall is one of the most common elements, but also the most standardised and bland. There is potential to better utilise these spaces as columbaria.

+ As the spaces are already walls, little grading is necessary to convert them.

- Because the location and orientation of the retaining structure is quite specific, there is little potential for spatial adjustments.

- These walls often border directly on to inappropriate land uses.

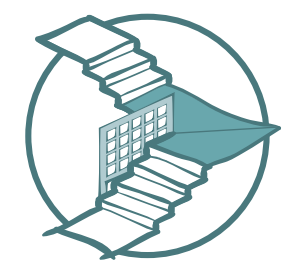

Public Stairways

There are often a large number of stairways bridging various gaps in the city's fabric too steep for conventional roads. However these stairways are often, much like access paths, underutilised.

( With multiple heights available, the total accessible surface area will generally be increased.

- Difficulty of access, particularly for those with impaired mobility.

$\Leftrightarrow \quad$ It is difficult for groups to stop anywhere but landings.

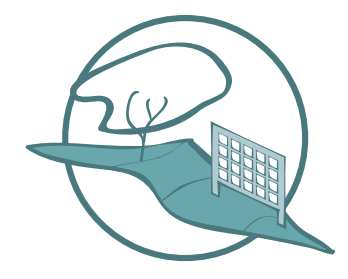

Triangle Parcels

Though a structurally strong shape, the triangle often proves to be difficult as a parcel shape. As such land parcels at the apex of acute angles in suburbs are often underutilised or not used at all.

( Because little total surface area is required for a columbarium, the constraints affecting building on these sites are less relevant.

- As these shapes are generally formed by roads, the site is likely to have multiple borders with the road.

- The periphery is likely to be developed as it offers a larger area. 


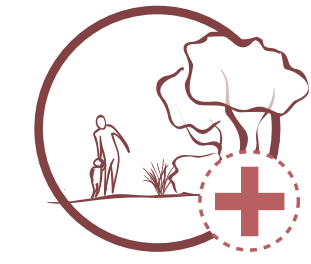

Park

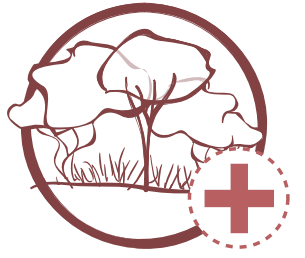

Reserve

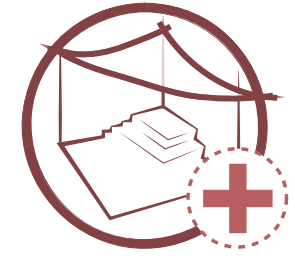

Square
Land Use Interactions

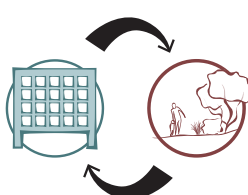

- The 'park cemetery' is a common and acceptable integration. Due to this the use of a Columbarium/Park integration would likely be more acceptable than less conventional combinations.

- However the inclusion of columbarium programme within the park is still likely to require careful planning in relation to other programmes within a park.
Added Value to Community

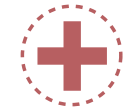

Other activities made available (ie. Petanque) through the variety of

programmes characteristic of modern parks.

Reintroduction of planting and breathing space in to the suburban environment.

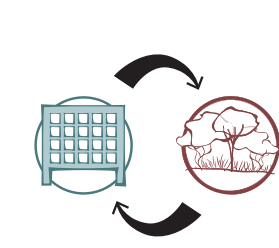

- Conforms to the "woodland cemetery" trope, once again making its acceptance more likely than other combinations.

Is unlikely to result in anything resembling a functioning ecology, as the space for the dead would be likely to replace the ground level and understory vegetation while retaining the tallest trees.
Added Value to Community

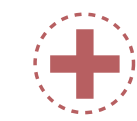

- Increased in experiences of birdlife and nature through the opening of

access to the reserve to the public.

- More opportunity for recreation within the reserve area.

- A more passive environment allowing for better intimacy
Land Use Interactions

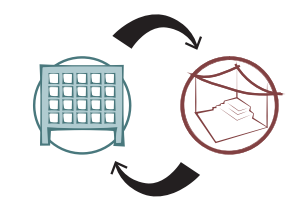

- Because of the emphasis on publicness' within square places, creating an intimate atmosphere may be difficult. However establishing a clear layered progression between two atmospheres is likely to be easier.

- Squares often find themselves particularly full when events such as markets and festivals occur. This would further affect the columbarium's atmosphere.
Added Value to Community

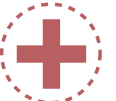

- A meeting place with a broad range of programmatic possibilities/

- Often the most open public places within community, allowing for a sense of freedom of expression during commemoration. 


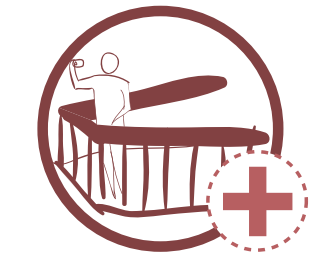

Viewing Platform

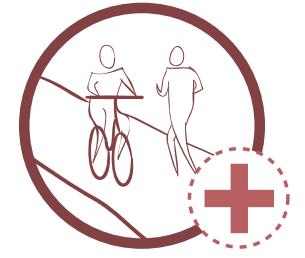

Walkway
Land Use Interactions

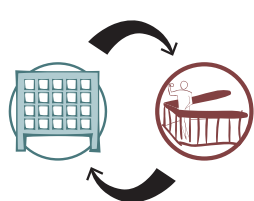

- The exposed nature of most viewing platforms mean that issues around privacy and intimacy for the columbarium may arise.

- The necessity of elevation for viewing platforms is likely to increase vertical surface area for the columbarium.
Added Value to Community

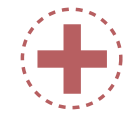

Access to better views of the suburb surrounding environs from public

spaces within the suburb.

- Area for relaxation and contemplation.
Land Use Interactions

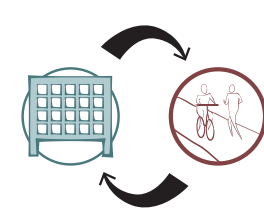

- Constant thoroughfare means that the placement of the columbarium must be carefully considered both in terms of potential obstruction of walkway users and privacy for columbarium users.

- Linear nature of walkways means expansion to meet increased demand for columbaria is possible.
Added Value to Community

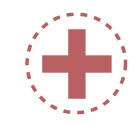

- Allowing for connections between important community nodes and easier transport in and out of the community core.

- Provides recreation and potential spaces for other programme.

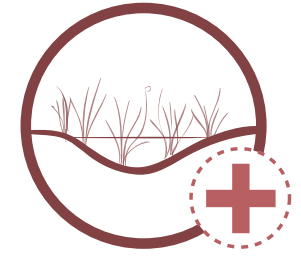

Stormwater Treatment
Land Use Interactions

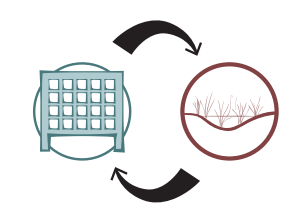

- Flowing water is an important aspect of many culture's mortality beliefs and practices.

- Water sensitive urban design generally follows topographic patterns, and as such the design of the columbarium will be influenced by them.
Added Value to Community

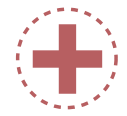

- Provides cleaned storm water within the suburb's limits rather than at the end of a pipe.

- Daylights the treatment process (while also daylighting death) 


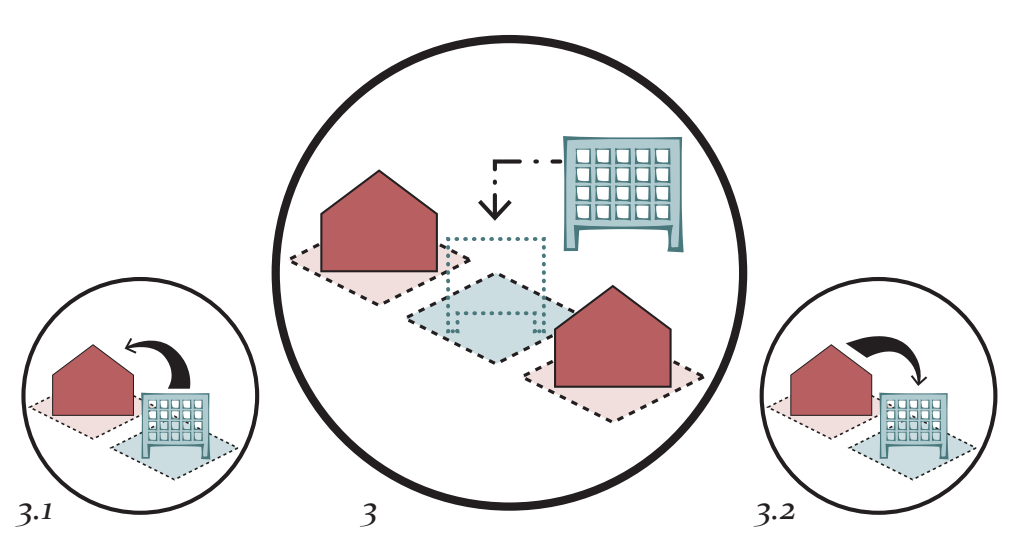

Infill Impacts

Taking into account the impacts of spaces for the dead on the surrounding land uses (2.1) and the impact of these land uses on the space for the dead (2.2) when selecting a site.

The act of infill with this particular land use means that the implications of and for the adjacent land uses must be considered.

This criteria involves assessing how the new columbarium might affect adjacent land uses and what effects it may experience from them. This section looks at several common types of land uses in terms of the affects and effects of a columbarium placed nearby. 


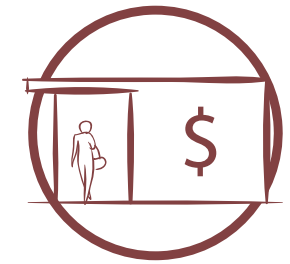

Commercial

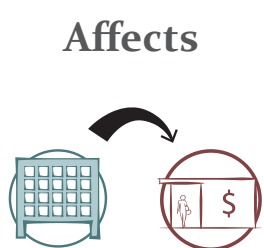

Worries about decreased business due to people's discomfort with death.

- Potential for increased business due to

columbarium patronage. Particularly certain types, ie. flower sales at dairies.

- Worries around health \& safety issues.

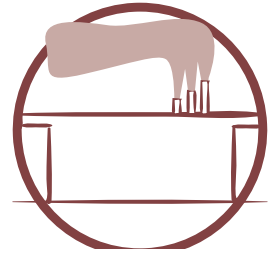

Industrial

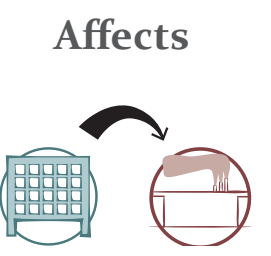

Too much nearby

commercial property may distract from the intimate and sombre ambience of a space for the dead.

- Large scale retail will be seen as inappropriate.
Increased nonindustrial traffic in area where low traffic volumes may have been relied upon in the

past.

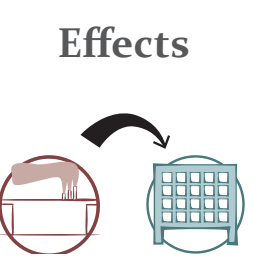

The atmosphere of

industrial land use is often one of strong efficiency and regimentation. This would act as counter to the atmosphere required for a space for the dead.

- Both noise and air pollution are common industrial issues which could be detrimental.

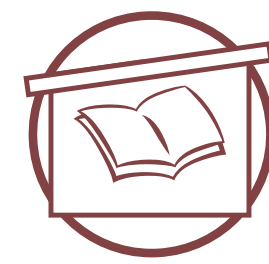

Library

Affects

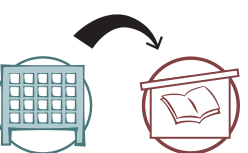

- With noise commonly regarded as the enemy of libraries, the noise generated in some

commitment ceremonies may interrupt readers.

- As libraries and columbaria both serve as civic archive of sorts, a level of synergy exists between the two uses.
Effects

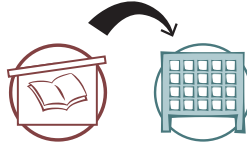

- Libraries generate high volumes of pedestrian traffic, which may lead to many passers through who, if uninvolved, may be detrimental.

- However this may also lead to greater community involvement, particularly considering the older user group common to libraries. 


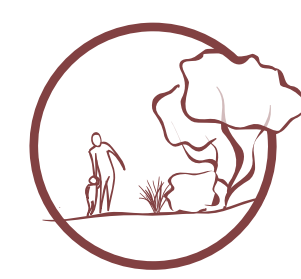

Park

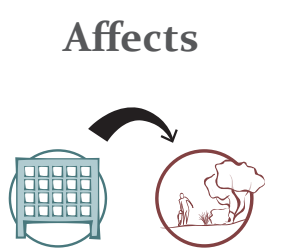

- People may choose to avoid the park due to discomfort with death, leading to an under-

utilized space.

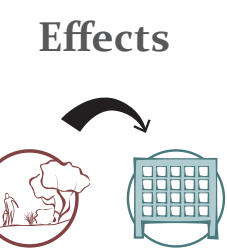

- Activities deemed appropriate in the park but not in the space for the dead may bleed across the parcel boundaries. ie.

Running, Eating.

- Events within the park may create noise and encroach on the space for the dead. ie. casual sport, music

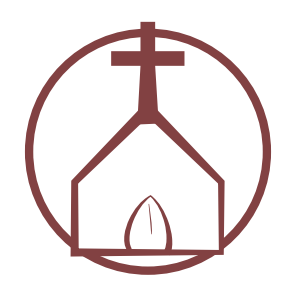

Religious Institution

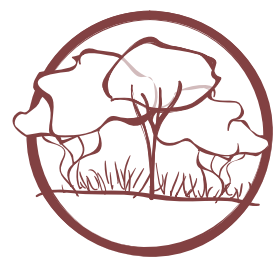

Reserve

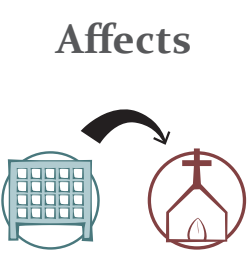

As religious beliefs often place significance on treatment of the dead, the support of the nearby

religious community may not be given.

Depending on the belief held by the institution particularly in regards

to cremation, the

institution's reaction to a proposed space for the dead could range widely.
Effects

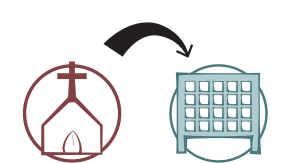

- If the institution'

community is to embrace the space for the dead, there is a danger that they may take sole ownership of the space, leaving it religiously nonspecific as intended.

- However a more acceptant form of custodianship should be encouraged.

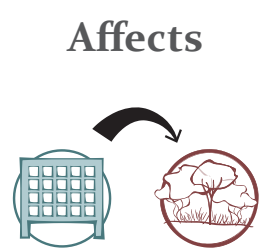

The inclusion of a collection of 'private' memorials on public / common land may be seen as encroachment.

- Fears that such a project might expand further limiting use of public land are also possible.

\section{Effects}

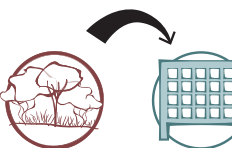

- Unintended

encroachments by reserve vegetation (which is often uncontrolled) on the space for the dead.

- Similar to parks, activities deemed appropriate for the reserve may not be so for the space. 


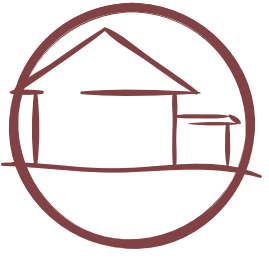

Residential

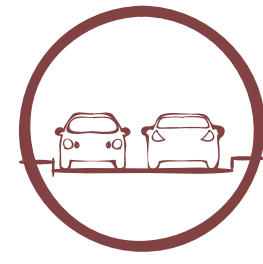

Road

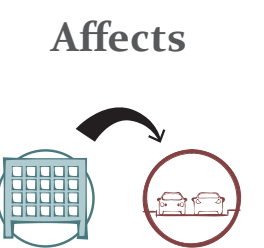

- Adjacent residential may give the feeling of a lack of intimacy / privacy within the space for the dead.
Potential improvement to drive-by scenery with a well designed space (and inclusion of any associated planting).

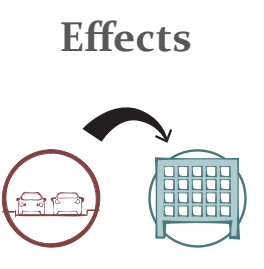

Noise from passing cars can be detrimental to the atmosphere.

- Road users may lead to a lack of intimacy within the space.

- The scale of the road (lanes \& volumes) will have a significant impact on their impact on the columbarium.

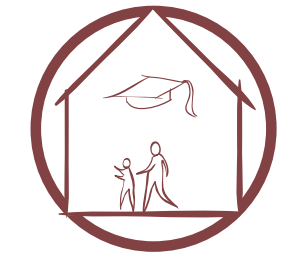

School

Affects

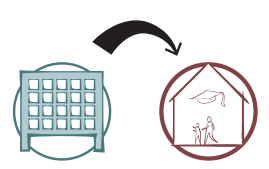

The feeling that children, who are commonly invocative of a sense of liveliness, should not be exposed to death means that parents may be

uncomfortable with this combination.
Effects

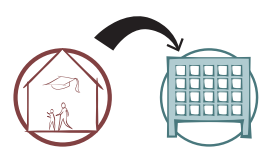

Schools can be very noisy places. This may detract from the columbarium's ambience.

- Children often

misunderstand what is

considered 'appropriate' behaviour around spaces of commemoration.

by increased use of

columbarium. 


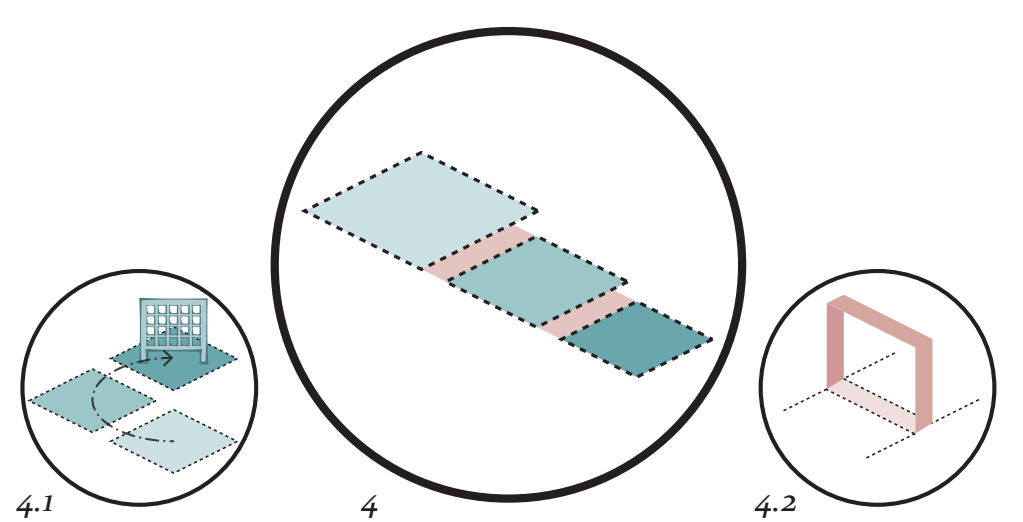

Layered Intimacy

Establish a sequence of distinctive spaces,

separated by clear thresholds, leading from public

to intimate and concluding with a space for

ceremony and interment.

The Columbarium space cannot sit directly adjacent to the public realm, the abrupt transition would have an adverse effect on the ambience of both environments.

Instead a spatial sequence leading from the public environment in to the intimate space of interment should be established. This sequence should involve a number of distinctive areas (4.1) which progress from one spatial condition to another. The thresholds (4.2) between these spaces must be clearly noticeable so that the user is aware of this progression from public to intimate and reacts accordingly.

This section addresses different ways of making spaces distinctive and forming clear transitions between them. It must be noted that these solutions can be used in combination. 


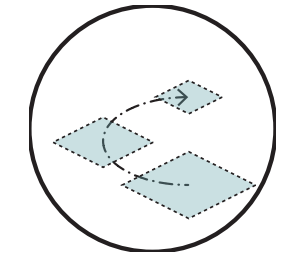

Size

This is the technique emphasised most by Alexander in A Pattern Language. He recommends the progression from larger to smaller spaces (334) as the closeness and confinement experienced in a smaller space links clearly with the intimate ambience.

However due to the space often required for commitment ceremonies, the point to which this size differential can be used is somewhat restricted, although the space for these ceremonies may also be located in a more outward layer than the columbarium.

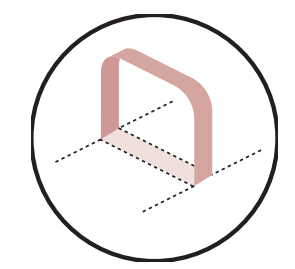

Portals

Though common the physical portal has functioned effectively as a marker of the threshold in many different projects.

The interpretation of what a portal is not necessarily restricted to the classical. In some cases the portal might even be implied by to separate freestanding elements, however if its presence is repeated at each instance of threshold between the spaces, its purpose will become clear.

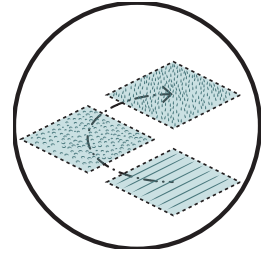

Area Surface
The groundplane has historically been of much importance to spaces for the dead. While the columbarium in some ways diminishes this, it can still serve an important role.

In order to make the spaces truly distinctive through surface qualities the difference should be clear. The use of haptic changes which extend beyond the visual will probably be of use here, for instance a transition from decking to gravel would mean a change in sound, feel and visual experience.

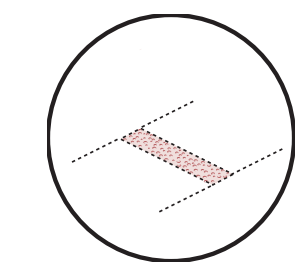

Threshold Surface

Surface can also be used to establish clear thresholds, although this is probably best used only when it is not already being used to establish distinctive spaces.

In this instance, because the change is only on the surface plane, the difference must be very strong. The surface area should be large enough that cannot be avoided while also being small enough that it will not seem to be a distinctive space in its own right.

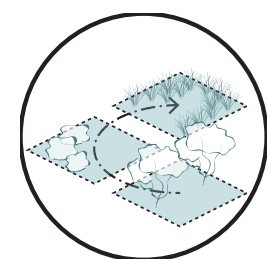

Plant Character

The characteristics of plants have long been used in spaces for the dead.

This change could be achieved either by making a microcosm of the transition through different ecosystems (eg. forest to grassland) or by focusing on one particular aspect of a plants characteristics (eg. wet to dry, tall to short). These differences can have a large impact on the ambiences of each space, helping to emphasise the transition from public to intimate.

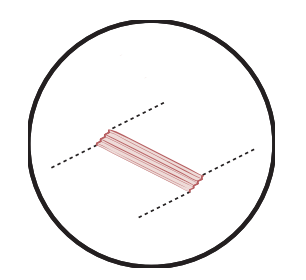

Elevation Change

A step or short ramp, used to change the height from one area to another, can be an effective threshold, because it is so noticeable.

Accessibility should be taken in to account here, as columbarium spaces are likely to need universal access considering the age of many of their users. If the ramp must be particularly long, it may even be worth treating it both as a distinctive space (through one of the techniques mentioned above) and as a threshold between two elevations. 


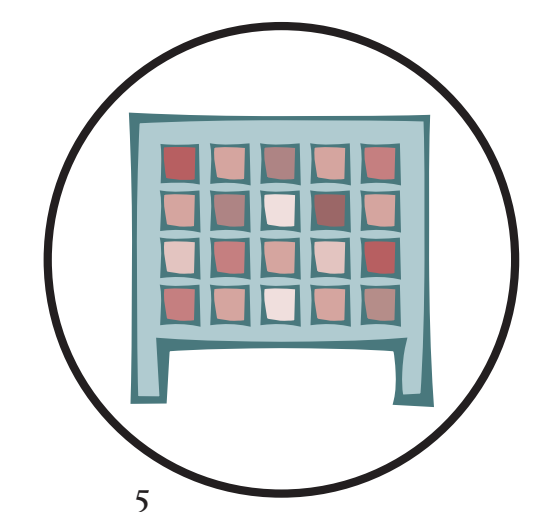

Individualised Interment

Allow for individualisation within the

commemorative spaces without compromising the design's overall integrity.

The need for more personalised options in death is a trend which must be addressed within this thesis. There is a fine line between enabling customisation and clutter. While it is important that people have access to a level of individualisation, it is also

important for the other users of the space that this does not

adversely affect the ambience of the space

This section looks at potential options for offering

personalisation to columbarium users, assessing them in terms of their value to those seeking to individualise, and also their potential to clutter and disrupt the overall design. 


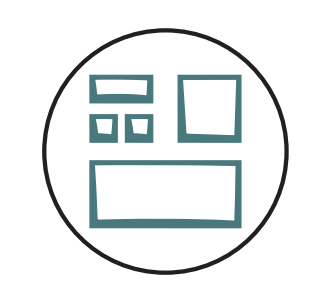

Niche Size Options

By offering varied sized of niches different preferences in terms of space for commemorative objects, and even multiple urns can be accommodated.

If too many different sizes are offered this option can cause a level of visual cluttering. However when kept to just two or three size options it simply leads to a less rigid and uninteresting surface.

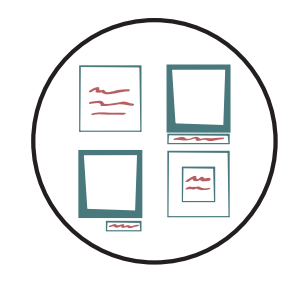

Inscription Options

The act of inscribing, on a metal or stone surface, the details of the deceased is a well established tradition. For columbaria this is sometimes done on the face of the niche cap, while at other times it is on a plate or slate placed near the niche. This is, at its most basic level, the type of individualization which is expected.

While in most cases these inscriptions are done to a set template, however if uncontrolled these inscriptions can contribute to cluttering, particularly when images are used.

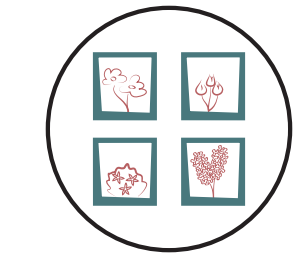

Decoration Options

Grave sites have long attracted flowers and other offerings, as do modern columbaria. This is because people like to be able to individualise the places of interment. While some columbaria encourage this, by placing ledges or receptacles near each niche, others do not, leaving friends and family only the ground at the base of the wall to place offerings.

There is clearly a potential for clutter here; however by accommodating the demand, the decorations can at least be organised in a way which aligns with the design intent.

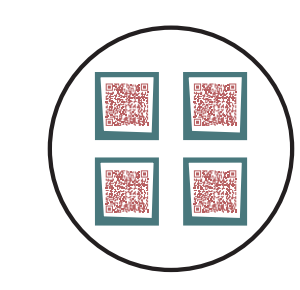

Digital Augmentation Options

This new development is intended to counteract the cluttering sometimes caused by peoples desire for individualization while providing more potential for individualization through technology. A QR code is inscribed, either stand alone or as part of a larger plate, and this links to a web page where messages, photographs and other similar things can be left.

There is almost no potential for cluttering, as the QR codes are very small, standardised elements.

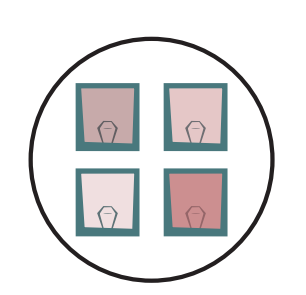

Niche Cap Options

While the niche caps in more traditional columbaria are opaque stone slabs, the trend in several more modern examples has been transparent and sometimes tinted caps. This allows light through the wall and a view in to the urn. It also allows a level of individualization, as the users can choose from a number of tints.

As long as the colour palette available is controlled, the effect will be that of a mosaic. However if complete freedom of choice is given, a cluttered and clashing result is likely. 


\section{Conclusions}

This chapter takes the main points established throughout the background chapters of the thesis and consolidates them in to five design principles. These principles serve as a framework for the reintroduction of space for the dead in to today's suburbs.

\section{The first three principles: (1) Informed Introduction,} (2) Infill Impacts and (3) Obvious Improvements are most applicable to the process of site selection found in the next chapter. However the second two of these are also, to a slightly lesser extent, applicable within the design process. The integration of a second land use of value to the community, for instance, requires a level of site scale design in order to be achieved effectively. And while the effects on the neighbouring and uses are identified and gauged during the site selection process, ways of mitigating them must be experimented with during the design process.

The final two principles: (4) Layered Intimacy and (5) Individualised Interment, both apply to design at the site scale, which is approached in the sixth chapter. The first deals for the most part with the larger scale spatial arrangement decisions to be made with the site, while the second concerns a finer detail series of decisions, particularly surrounding the detailed design of the columbarium wall itself.

While they are to be used within the following two chapters, it is important to note that these principles are also an output of this thesis. They are intended to be broad enough in implication that they might be used by others seeking to deal with how locate and design columbaria or indeed another types of space for the dead. The design principles also serve as the underpinning structure to the exegesis, where the resulting design is critically assessed based on each principle. 


\section{Site Selection}





\section{Introduction}

Shifting spaces for the dead back into the places where we live is a move likely to attract some form of controversy. Because of this it is important that the process of site selection does all it can to mitigate this controversy and ultimately gain the approval of the community for whom the spaces are set to cater. This chapter seeks to carry out this process of site selection for a network of columbaria across Wellington City.

Using the design principles from the previous chapter, site selection is preformed first at the city scale, identifying the existing system of spaces for the dead and how it will be augmented. The main suburban centres are identified and a plan for the phasing of the networks development is given.

Next, at the suburban scale, the community's core is identified and potential sites in this area are selected. This is done with an

emphasis on selecting sites which are underutilised, in order to provide obvious improvements to the community.

Finally, at the parcel scale, these potential sites are assessed based on the impact of their surrounding context, in particular the level to which they affect adjacent land uses, and what effects they experience.
In this chapter suburban public libraries have been used as an indicator of suburbs of sufficient density to justify the introduction of columbaria. As such the suburbs focused on are Brooklyn, Island Bay, Kilbirnie, Miramar, Newtown and

Wadestown, as these are the Wellington City Suburbs with public libraries. Karori is not included (although it has a library) as the cemetery there already contains a number of columbaria. This is not intended to imply that other suburbs without a public library lack the necessary local centres to justify intervention, it is simply an indicator of a suburb where the council has made an investment in developing the community, and a way of maintaining a manageable scope in a city of 43 suburbs.

The process of site selection in this chapter has involved the production of a significant quantity of material, with eighteen mappings having been made at the parcel scale alone. In order to simplify the chapter, five of the six suburbs site selection processes have been abbreviated into a purely graphic form, while the work around Brooklyn (one of the two locations used in the design) has been elaborated on fully. 


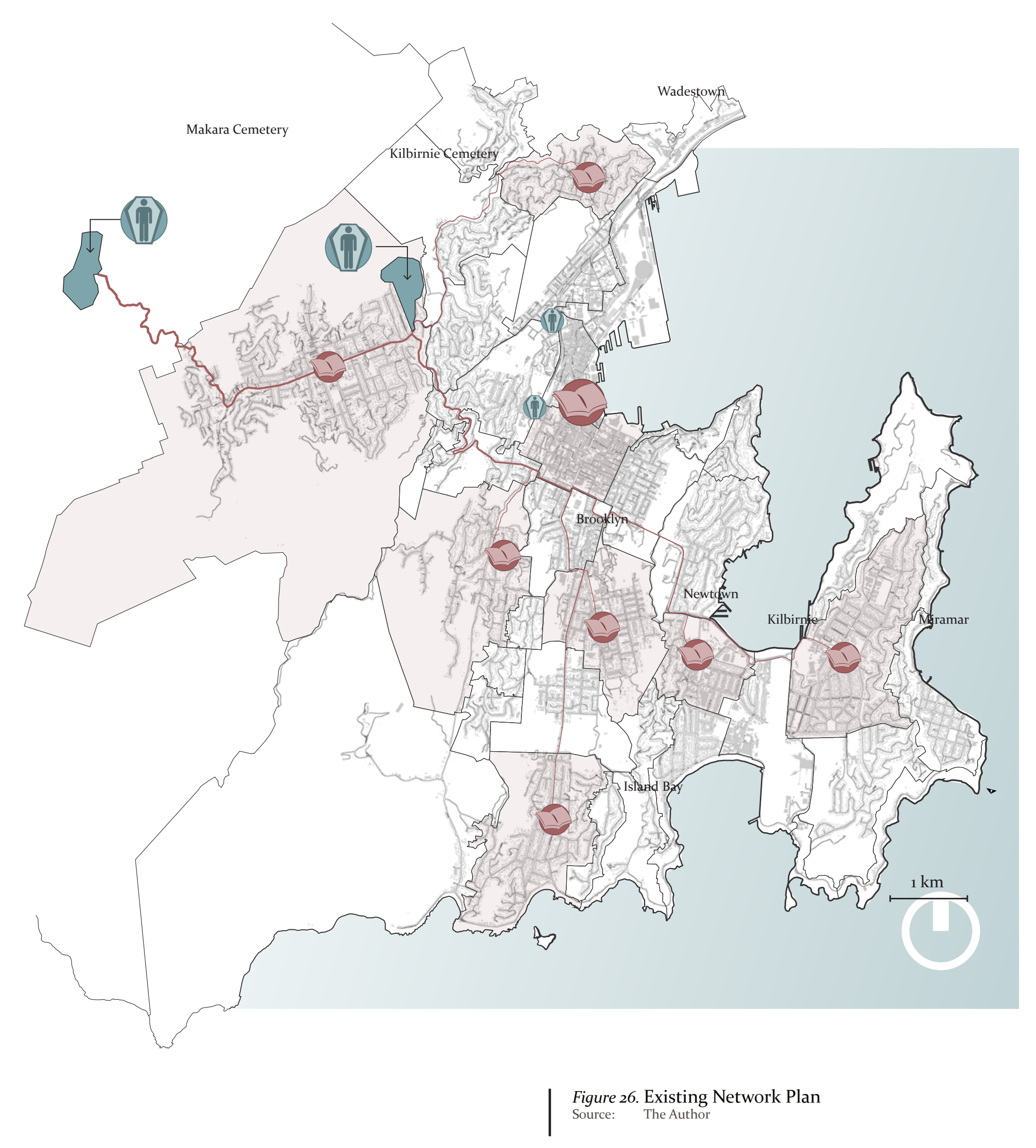




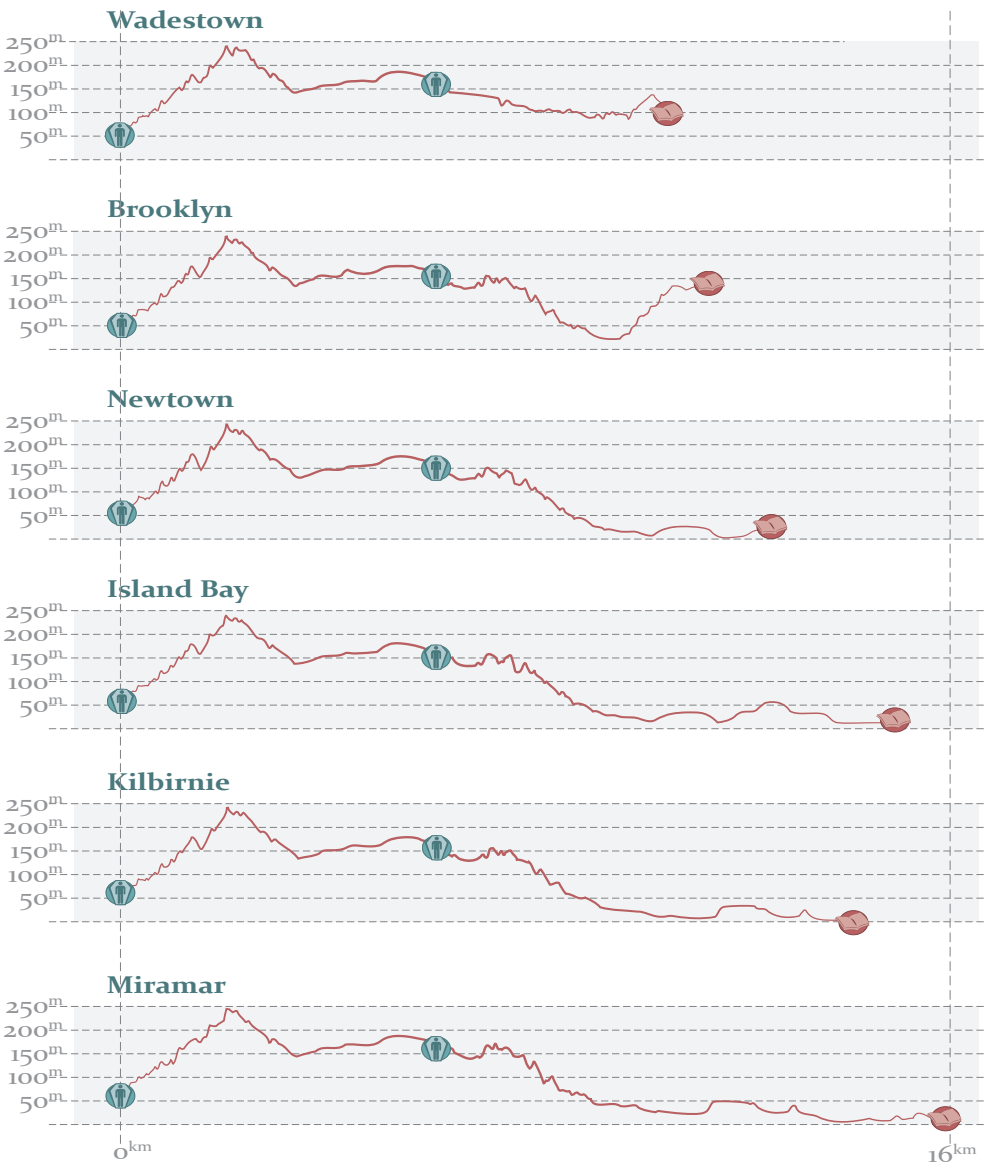

Figure 27. Suburban Centre to Cemetery Elevations

\section{City Scale Network - Existing}

The system of cemeteries in place within Wellington City reflects the city's expansion since European settlement. The Bolton St

Cemetery; now bisected by the Wellington Urban Motorway once sat at the north western edge of the city before the suburb of Thorndon enveloped it.

Similarly the Mount Street Cemetery, a catholic cemetery established around the same time, was also once located at the city's edge but has also been quickly enveloped by the university and city. However both these cemeteries have now been closed and are effectively 'memorial parks'.

The Karori Cemetery, established in 1891 (Cemetery Management Plan 5), was established in order to close the Bolton St Cemetery and move space for the dead further from the city. However it was quickly surrounded by Wellington's largest suburb, and by 1920 the demand for plots at Karori, combined with the relatively few spaces left, led to the establishment of the Makara cemetery. While the Karori cemetery is still technically open today, it is effectively full, with interment only being offered to those with family plots.
However Makara still has plenty of space for burial and has developed a range of different burial spaces to cater to the various cultural and religious groups within Wellington.

With this said, access to Makara is an issue. The drive out is anywhere from ten to sixteen kilometres (including at least $200 \mathrm{~m}$ of elevation) depending on which suburb one travels from, and for those wishing to commute by public transport, the only access is by a bus which travels there only once a month. So for those who can still drive, regular visits are a difficult task; for those who cannot, they are an impossibility.

At present Makara is effectively the only option available to residents of Wellington's suburbs, leaving those wishing to remain within the suburb in which they lived with little choice. It is these options which the columbaria network seeks to provide. 

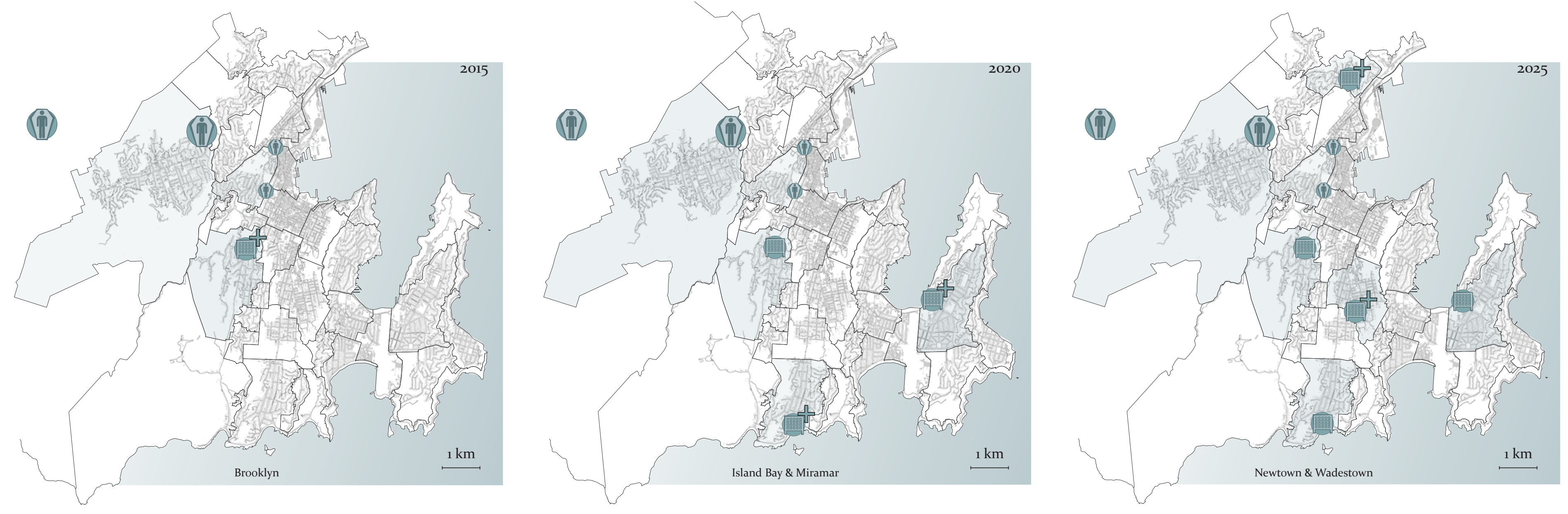


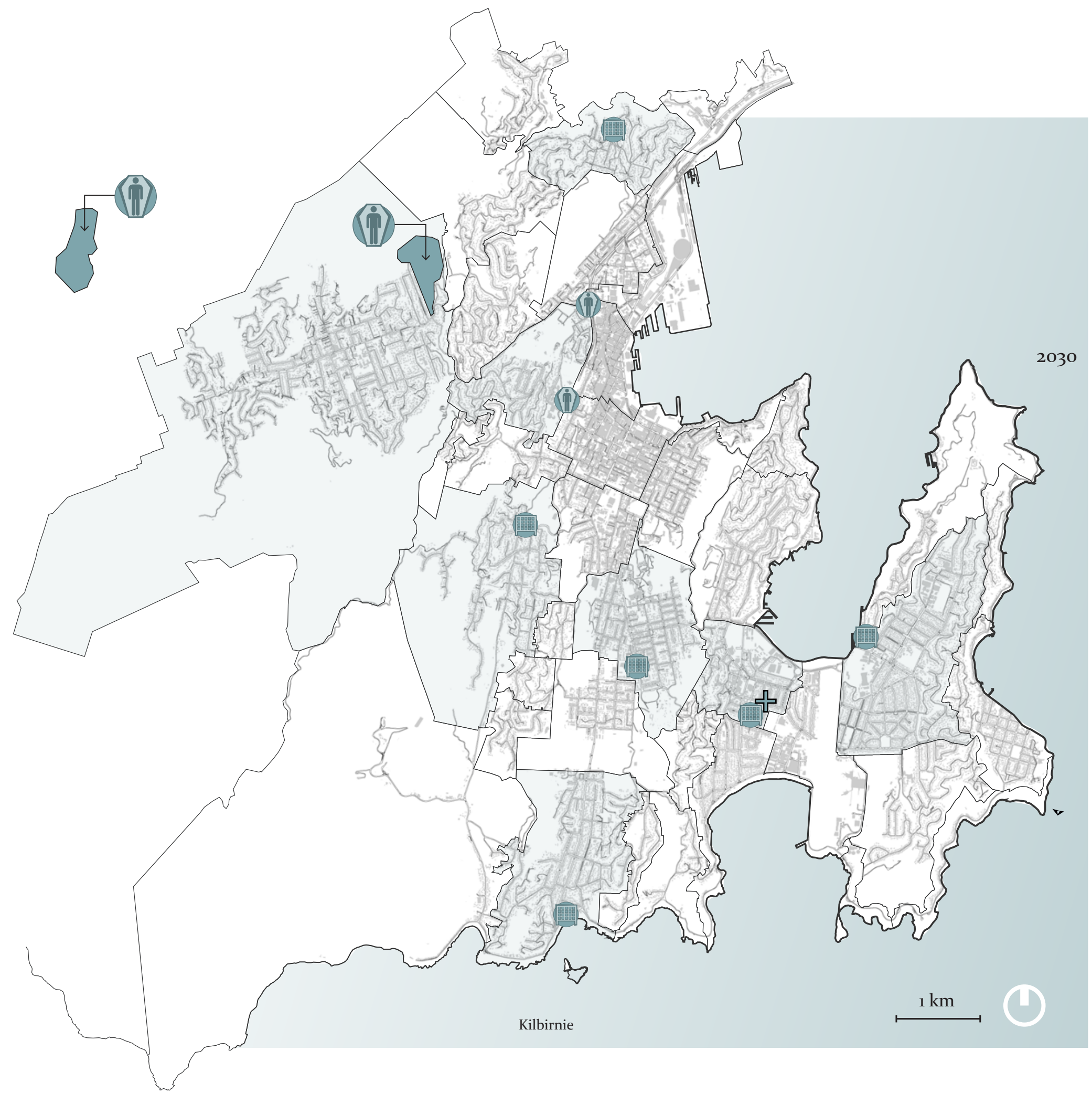

\section{City Scale Network - Proposal}

Because of the potential controversy around such a network, immediately establishing the entire network would be unwise, not to mention a large up front investment from the Wellington City Council.

Instead a phased approach is taken, looking to establis the network over 20 years. The first intervention would be a 'catalyst' project, based in the suburb of Brooklyn completed by 2015; this suburb is chosen as the 'testing' site due it it's high rate of secularity. Next the two suburbs with the longest distances to Makara: Island Bay and Miramar would be developed by 2020 . Following this the suburbs of Wadestown and Newtown with strong community organisations, which would probably be demanding a columbaria of their own having seen the first three, would be developed by 2025. Finally the suburb of Kilbirnie,

which is earmarked for a medium density development creating six hundred new dwellings by 2031 (McIndoe et al. 26), will have a columbarium developed by 2030 to align with this development. 
Built Form Density

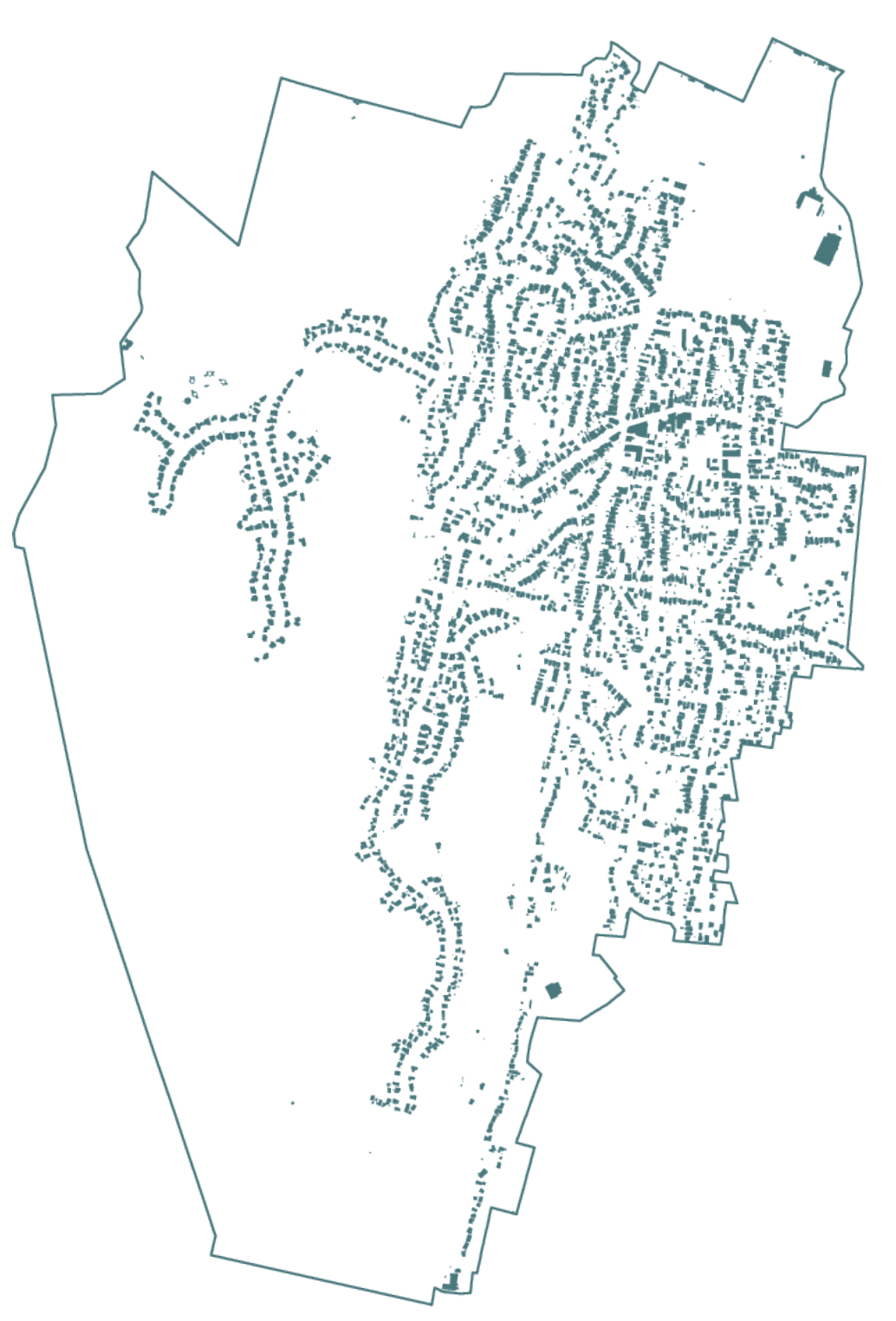

Topography

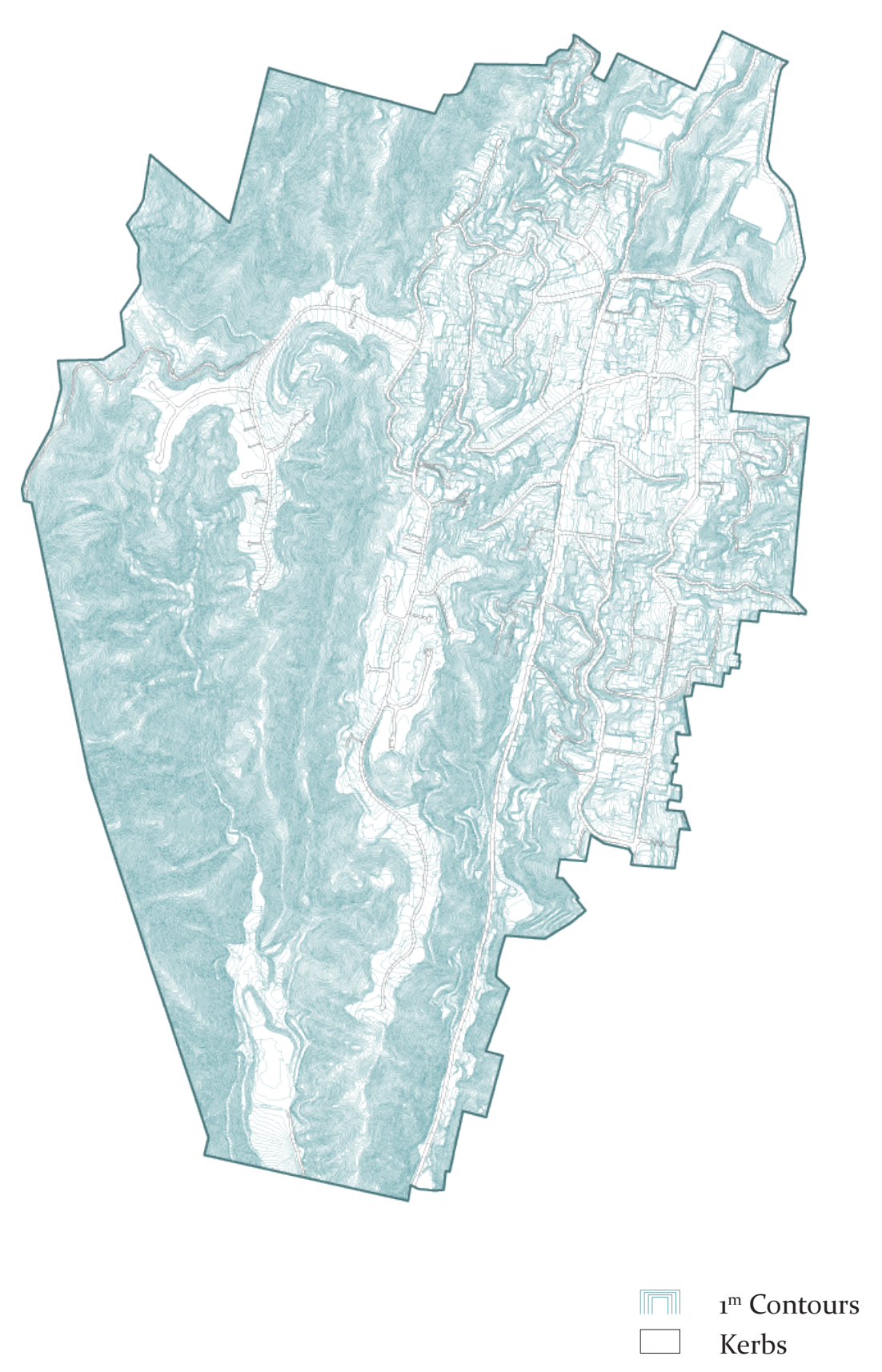

Circulation

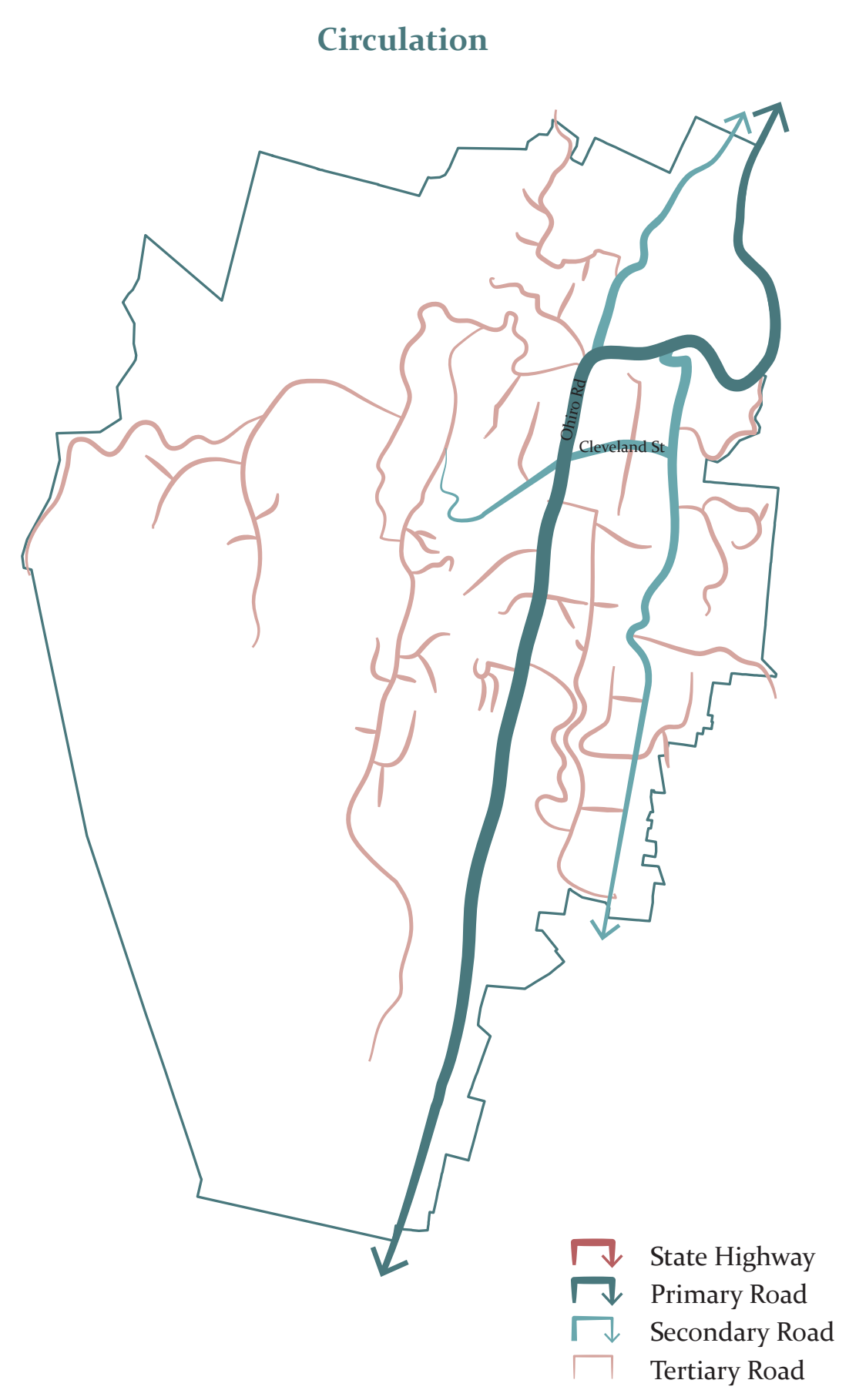


Land Use Type

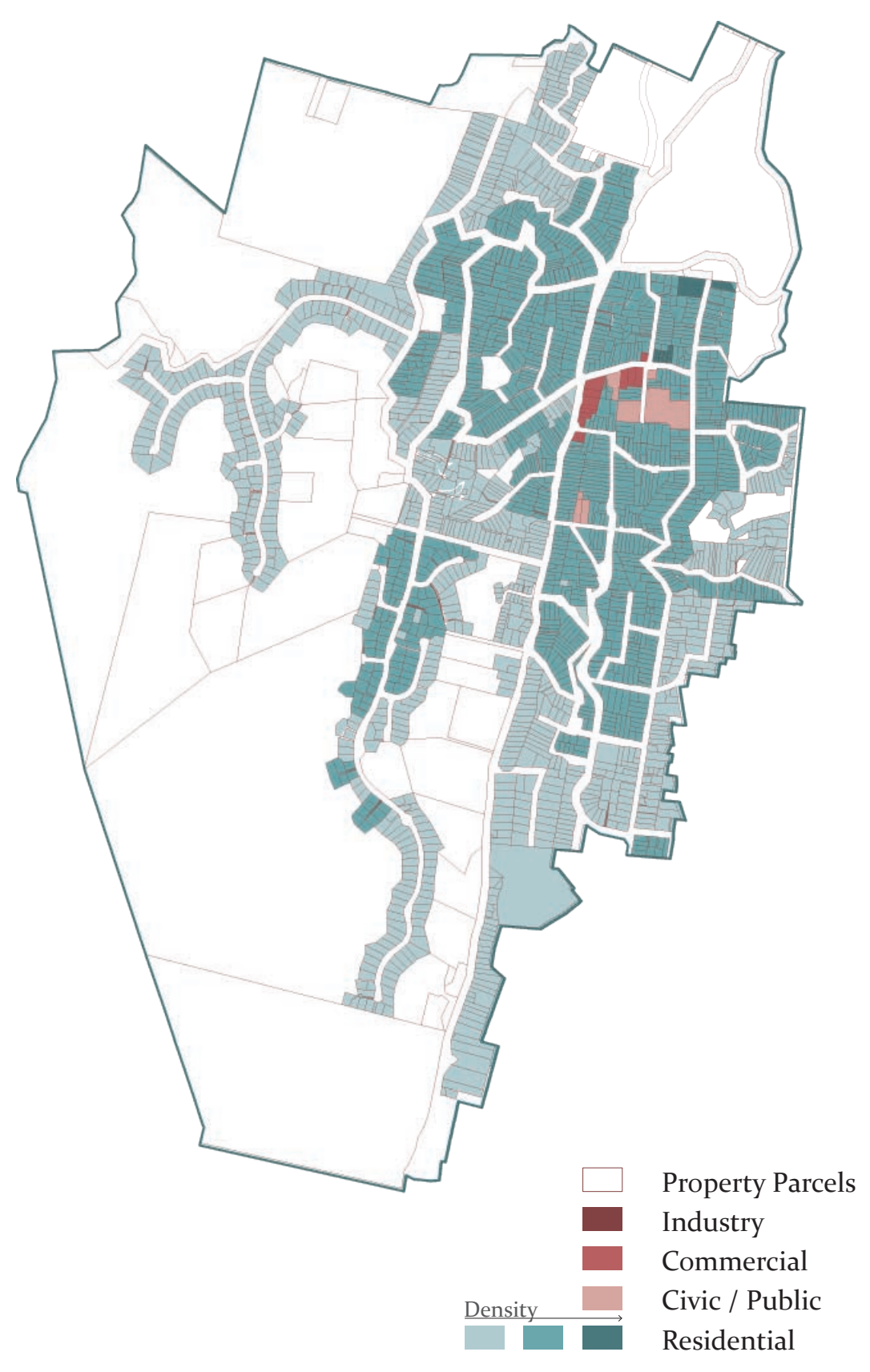

Suburban 'Core'

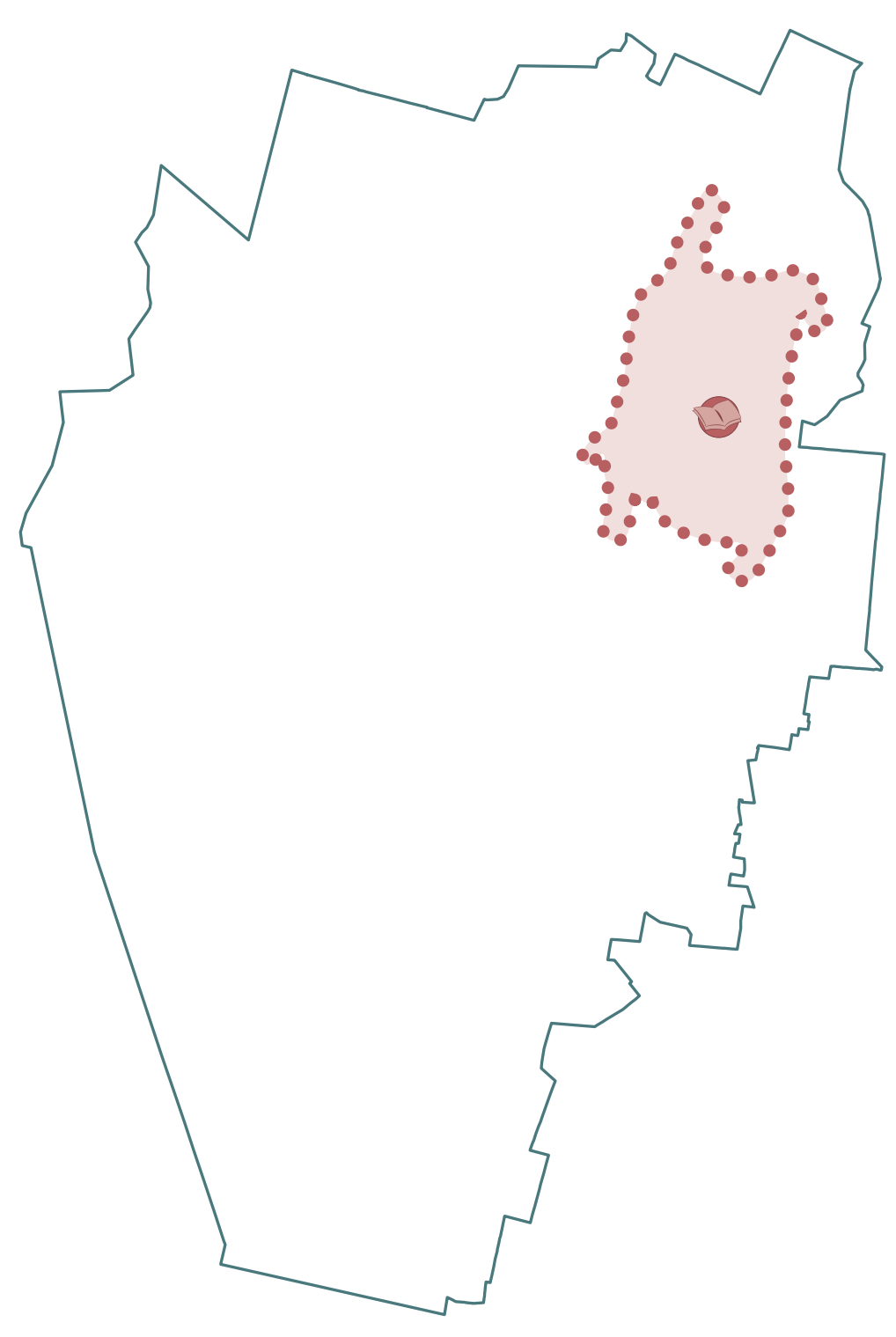

$\because$ Community Centre Area

- Suburban Public Library
Application of Informed Introduction Principle

The Suburban boundaries of Brooklyn are large, however the majority of the actual built density is concentrated on the

eastern side. This is due to the steep topography on the western side. In addition to this the actual population density is likely concentrated in the north west, as the dwelling density to the north is higher.

The primary road (Brooklyn/Ohiro) feeds from both the suburb and several of it's surrounding suburbs in to the central city. As such it carries high volumes of traffic, both vehicular and pedestrian.

The string of shops and civic buildings such as the library and fire station running along Cleveland St form what is effectively the community centre, with the two schools sitting a short distance away.

In layering this information, the suburban community core area is based around the corner of Cleveland St and Ohiro Rd but is skewed to the east and is stretched along the primary road in to the city. 

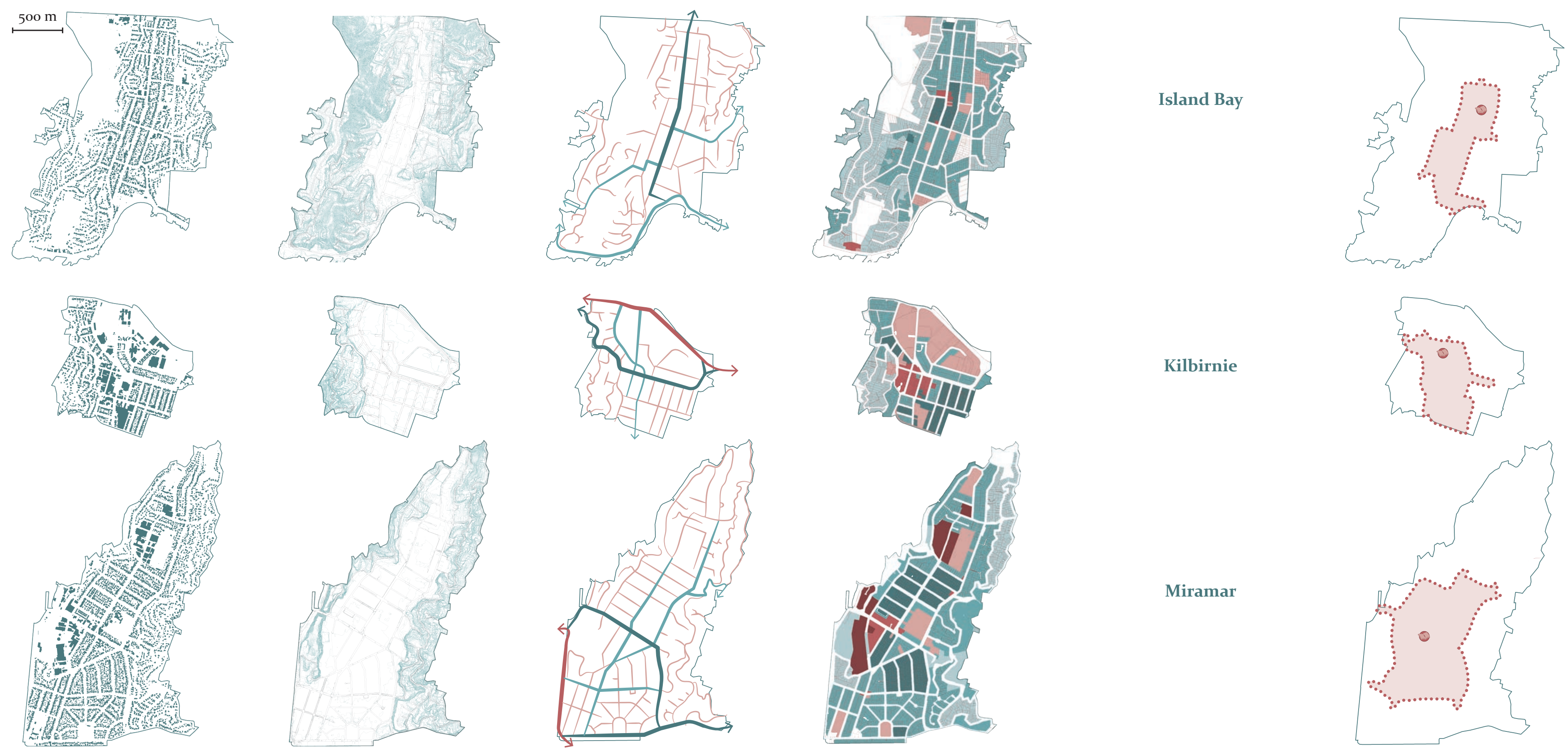

Kilbirnie

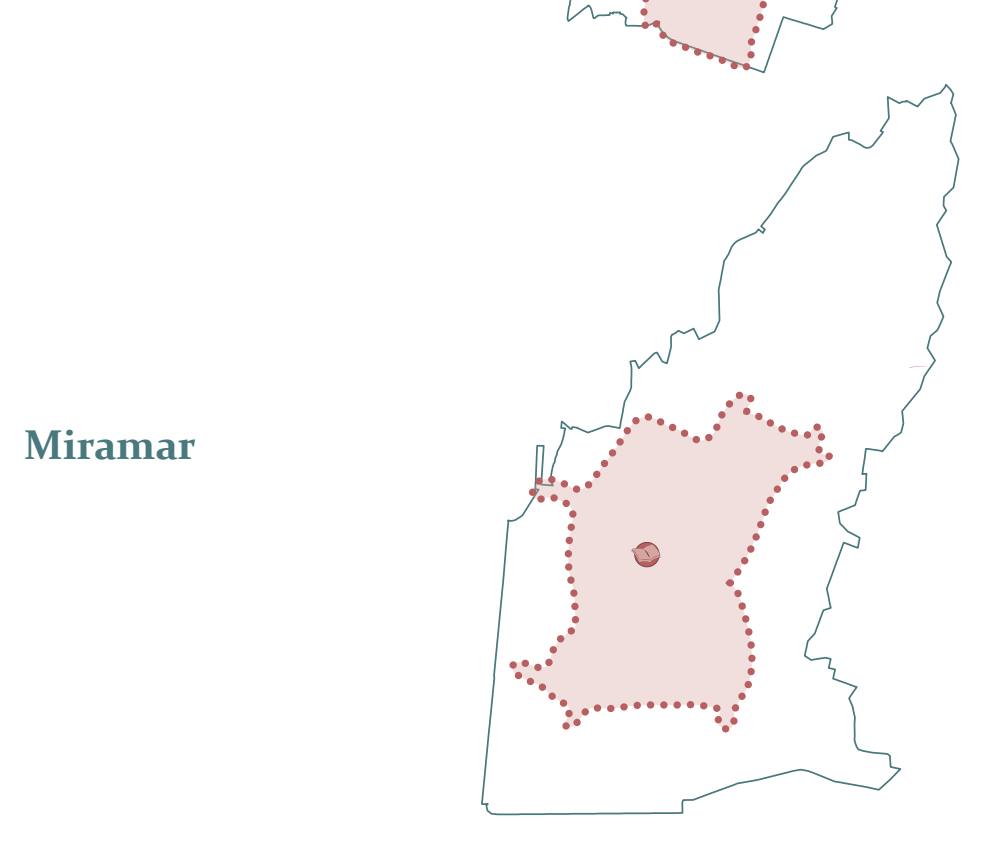

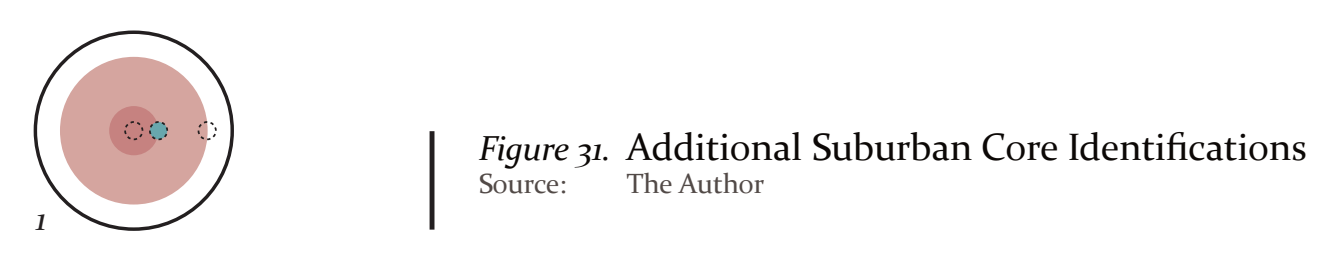



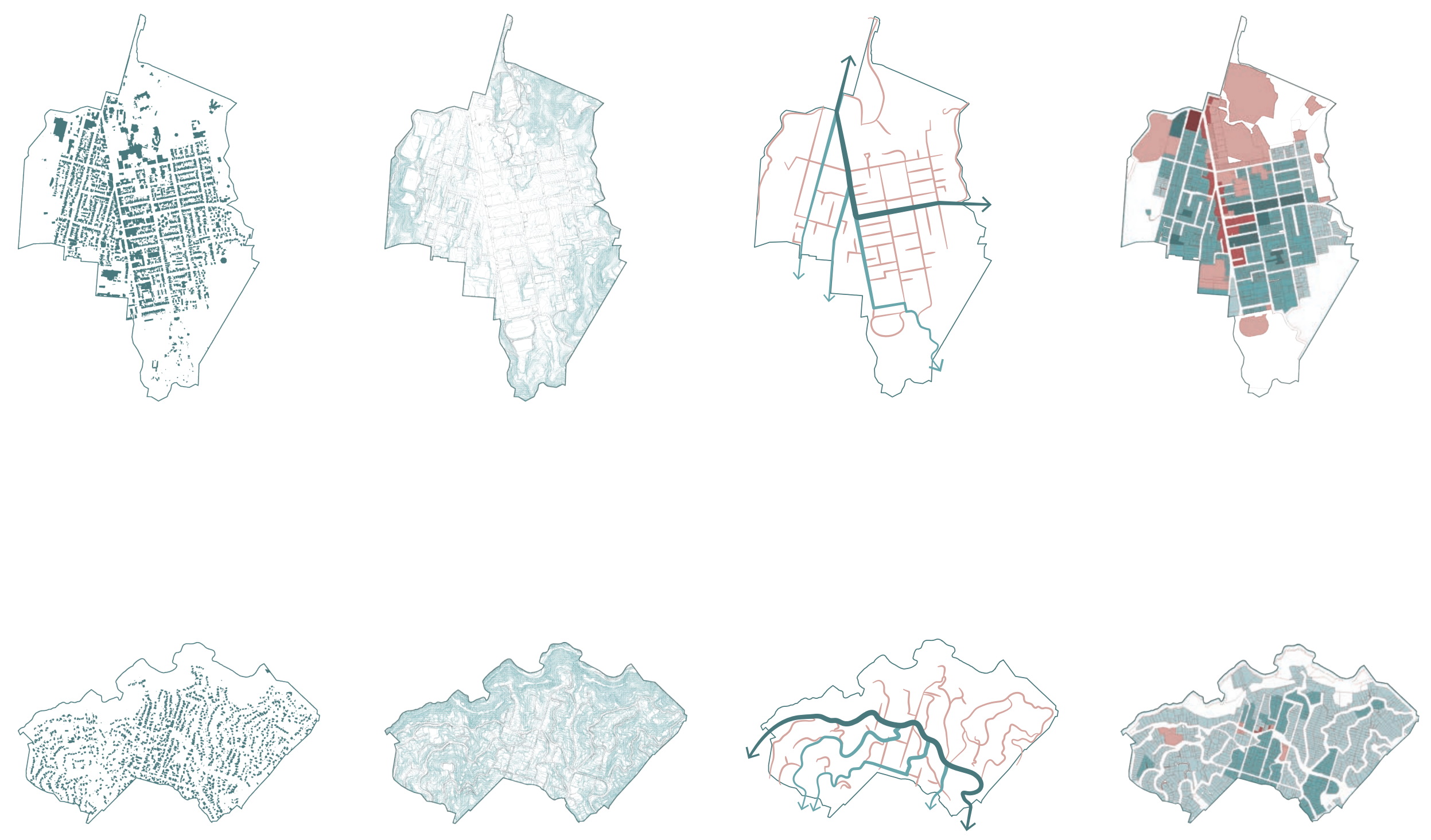

Newtown

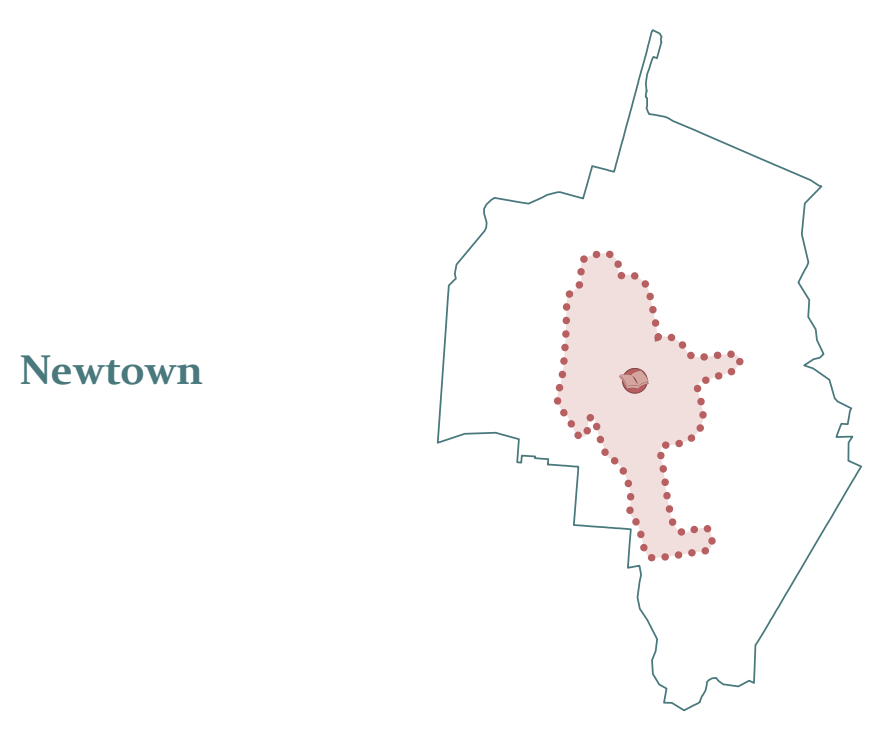

Wadestown

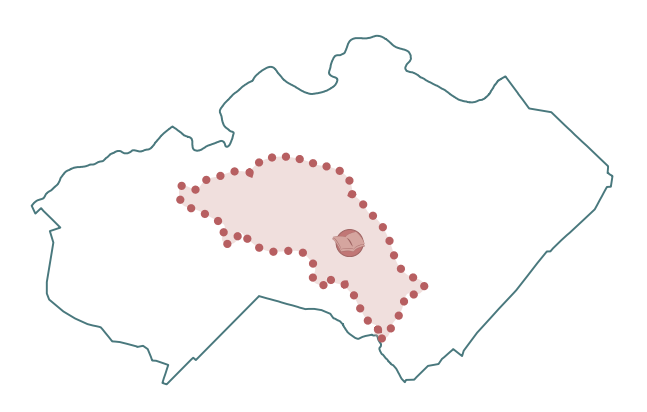


Application of Obvious Improvement Principle

Site A is quite far from the community's centre but still sits within the core, due to the importance of the roads which it sits between

The site offers some of the best views of the Harbour available from a public space in Brooklyn.

The large triangular planting bed currently occupying the site restricts views while the seats backing on to it are poorly used and are visible from few surrounding points.

Site B sits slightly closer to the community centre and still aligned with the primary road.

It sits just across the road from the current northern entrance to Central Park.

A reasonable steep slope plus it's triangle / road margin parce mean that it is currently unused, despite its good location.

Site C effectively sits in the community centre, on Cleveland St next to the library. This is perhaps a little too close under Informed Introduction (1) however it is still a block from the most major commercial areas.

The bus stop and turning bay are almost redundant and in particular the "traffic island" at the centre is poorly utilised.

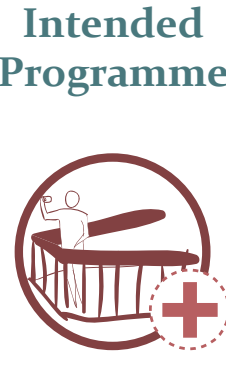

Viewing platform

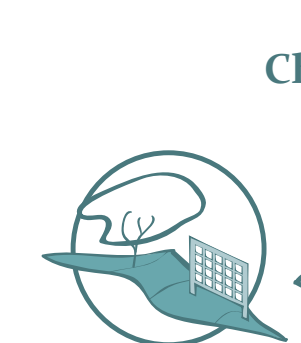

Triangle Site
Spatial

Characteristics

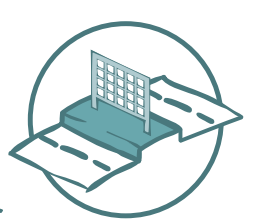

Road Margin

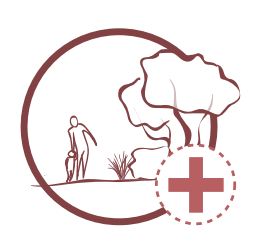

Park

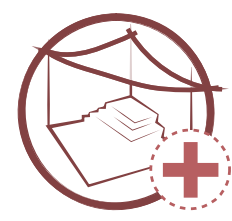

Public Square

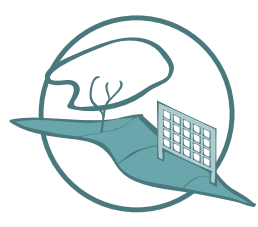

Triangle Site

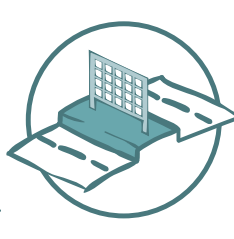

Road Margin

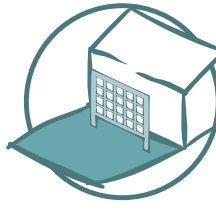

Building's Edge

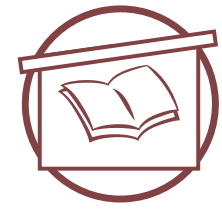

Library

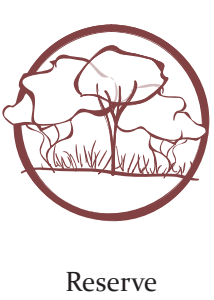

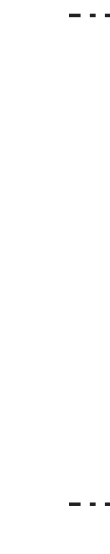

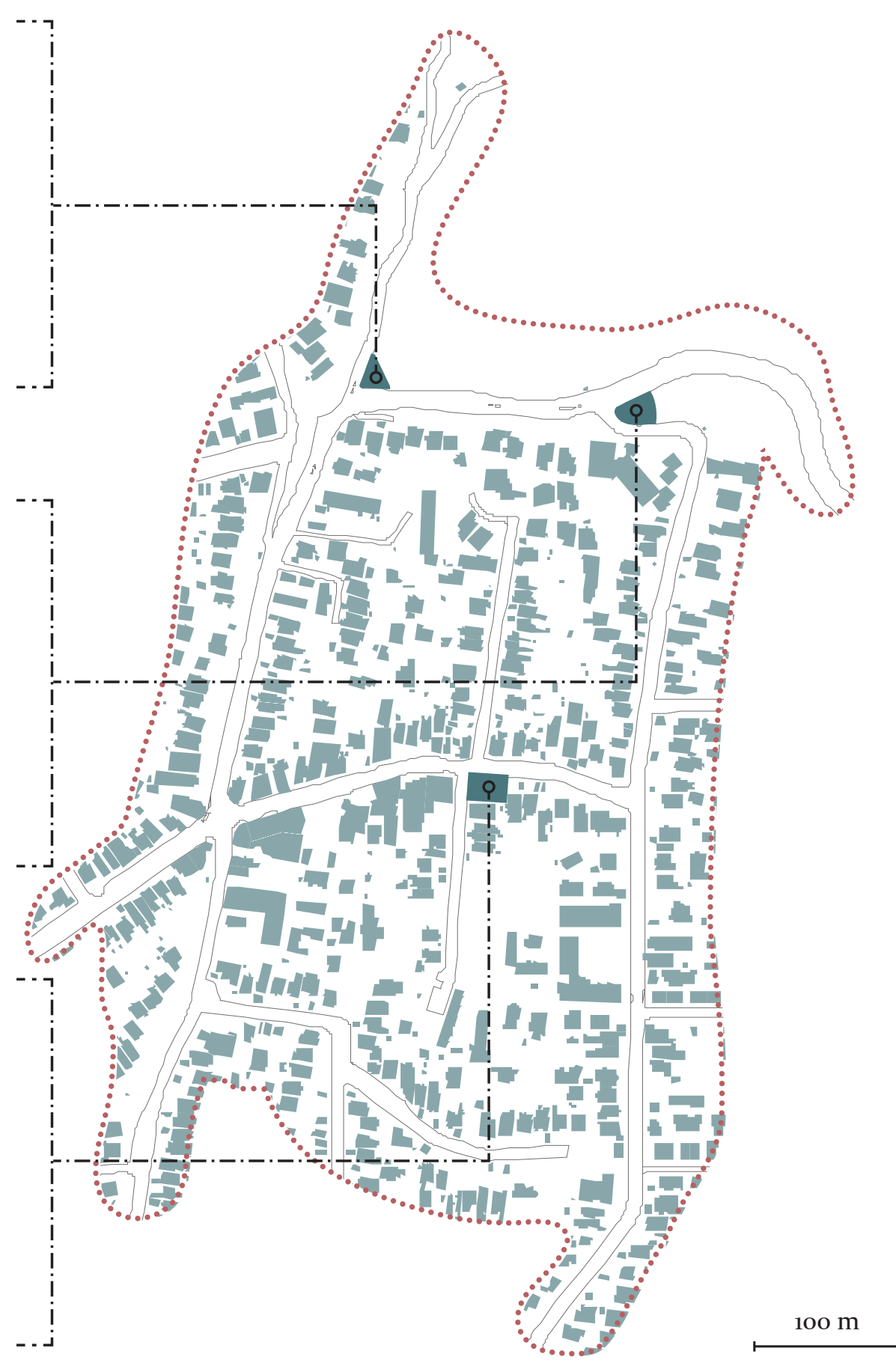

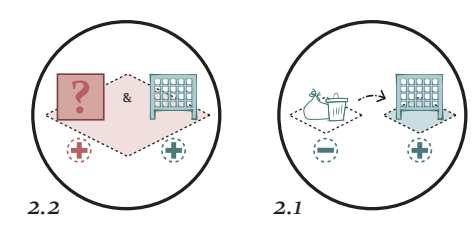


Island Bay

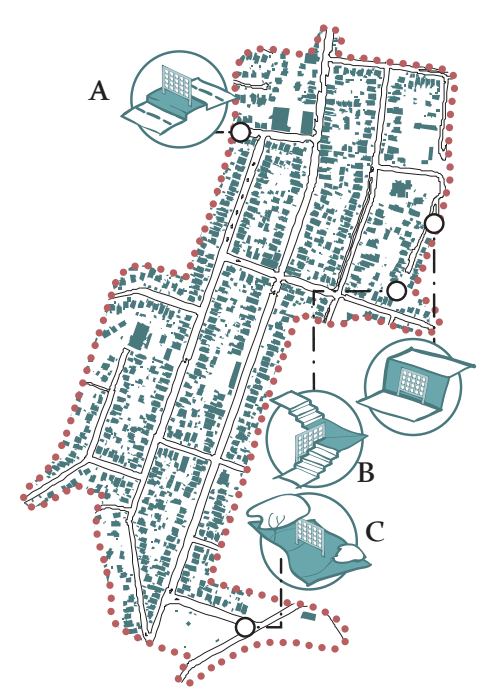

Kilbirnie

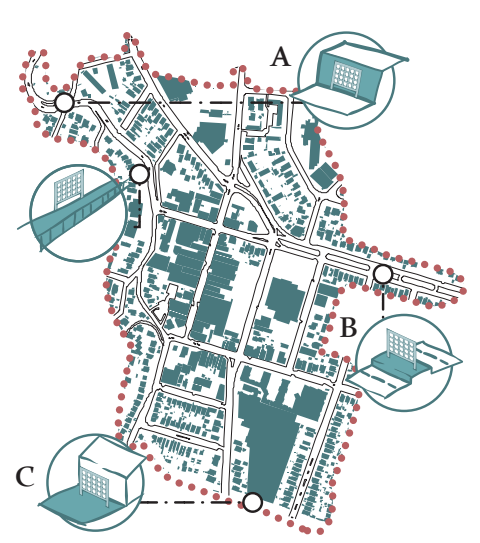

$\stackrel{200 \mathrm{~m}}{\longmapsto}$

Figure 33. Additional Suburbs Site Identification

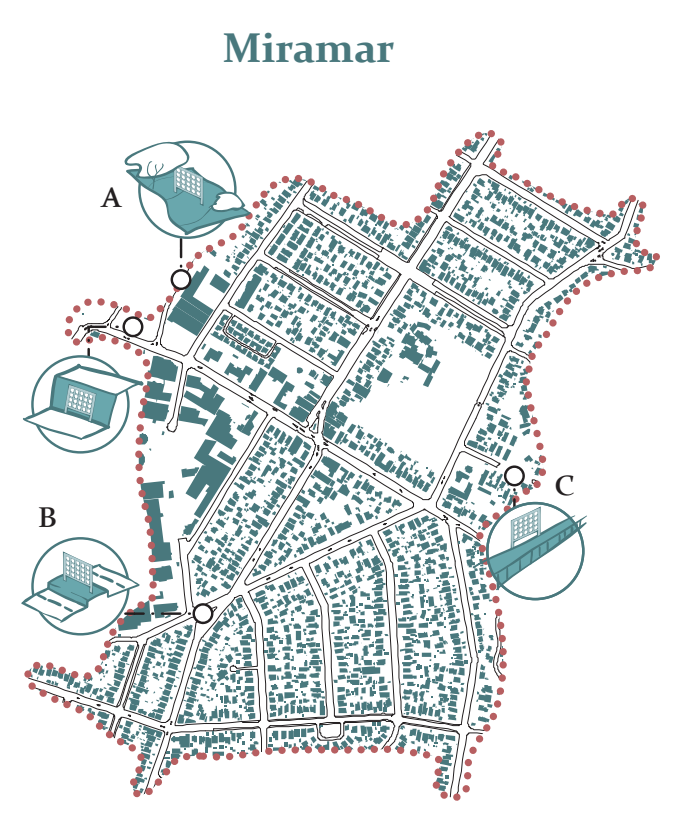

Newtown

5

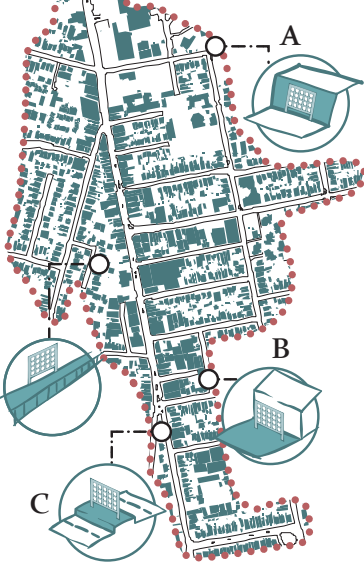

Wadestown

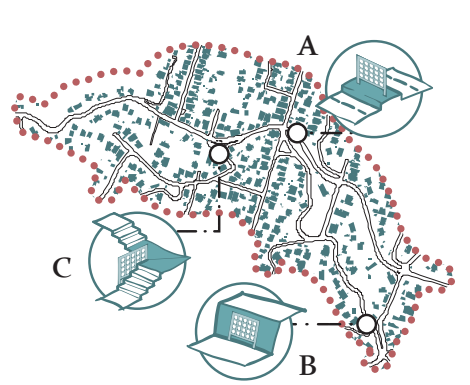


Application of Infill Impacts Principle:

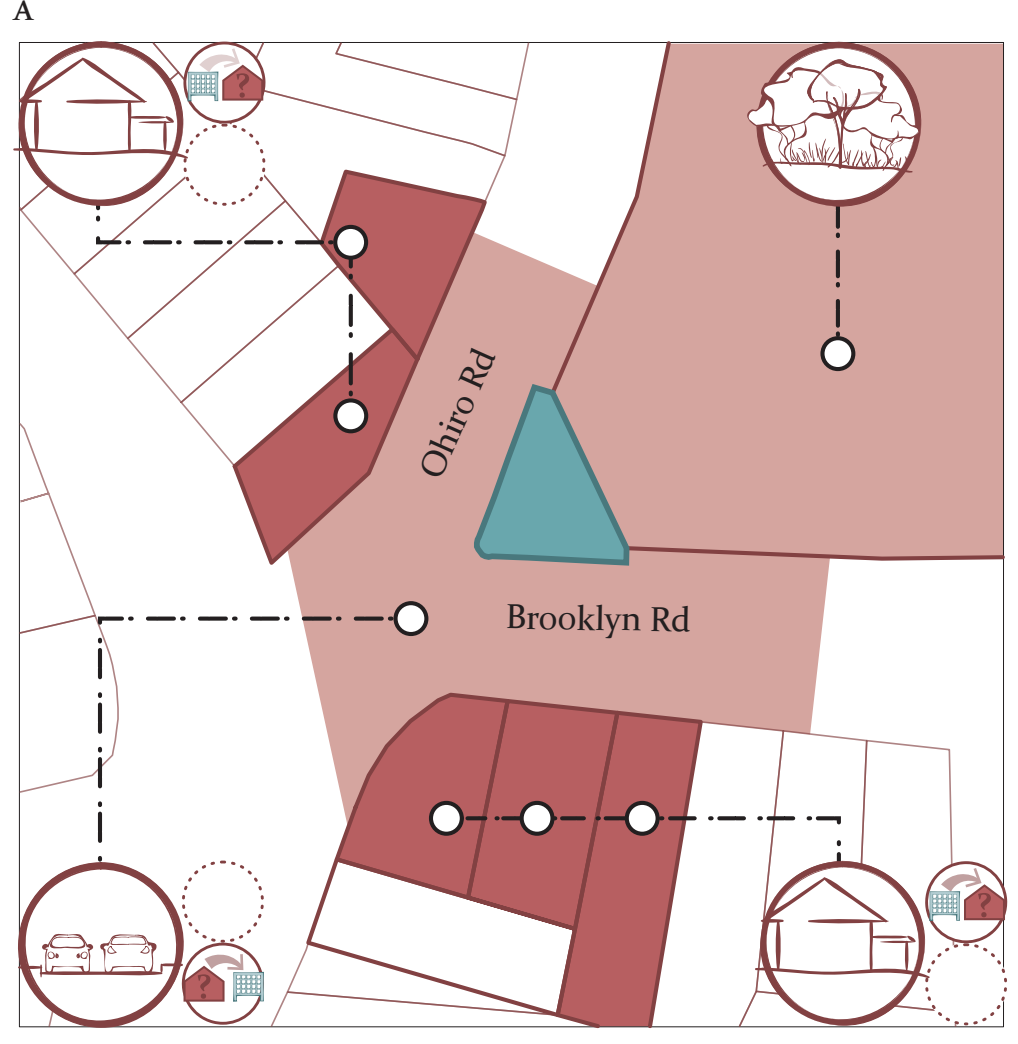

- The apartment building to the south of the site has the most potential for impact on the columbarium, and will require mitigation.

- Other surrounding residential is, however, effectively unimpacted due to the major elevation differences.

- The businesses of Brooklyn Rd in particular, may lead to noise and privacy issues.

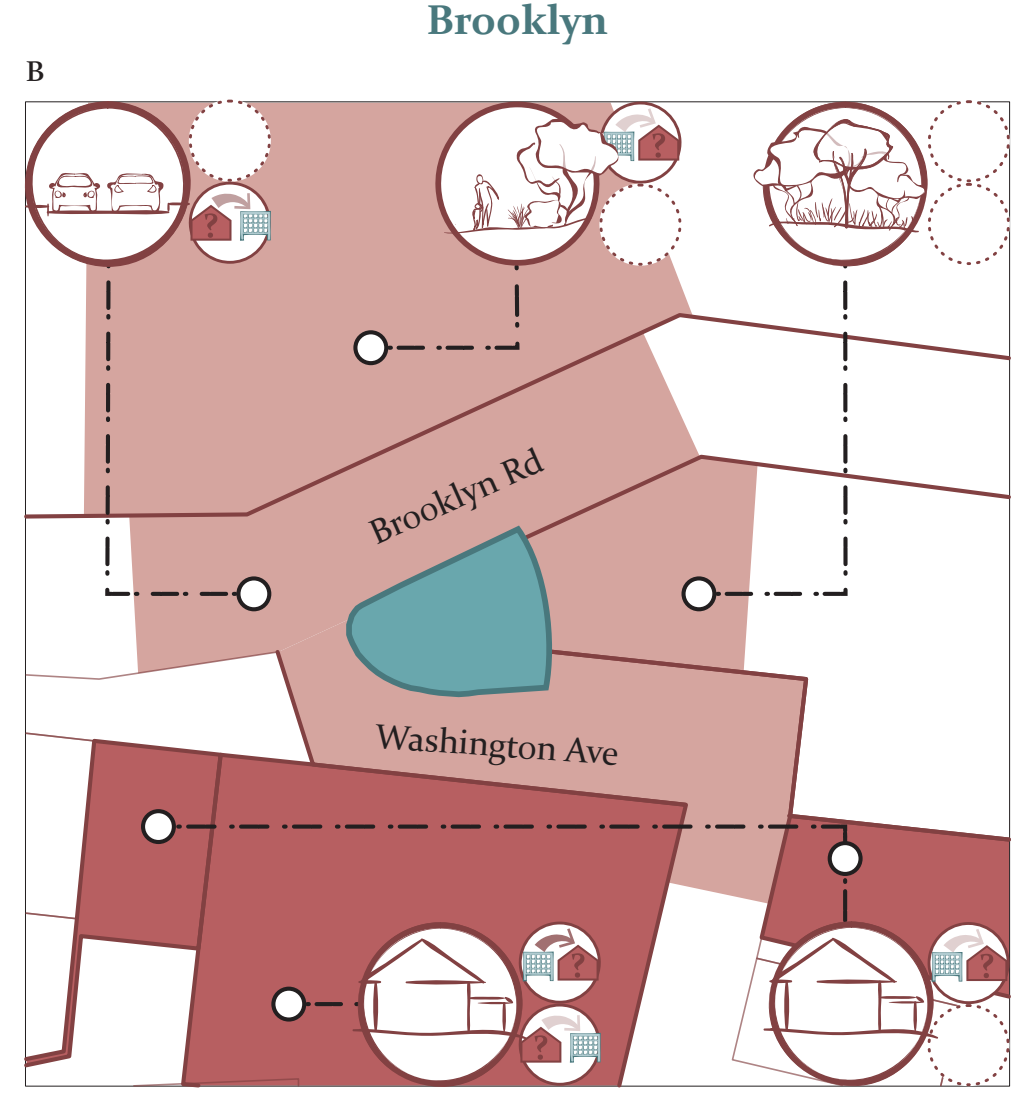

- The connection with central park will be one of the most important aspects of a design for this parcel.

- Brooklyn Rd will have a similar effect as with site A.

The Malay Embassy residences across Washington Ave would need to be taken in to account, and consulted, before the site could be approved. Careful measures to mitigate any potential impacts would be necessary.

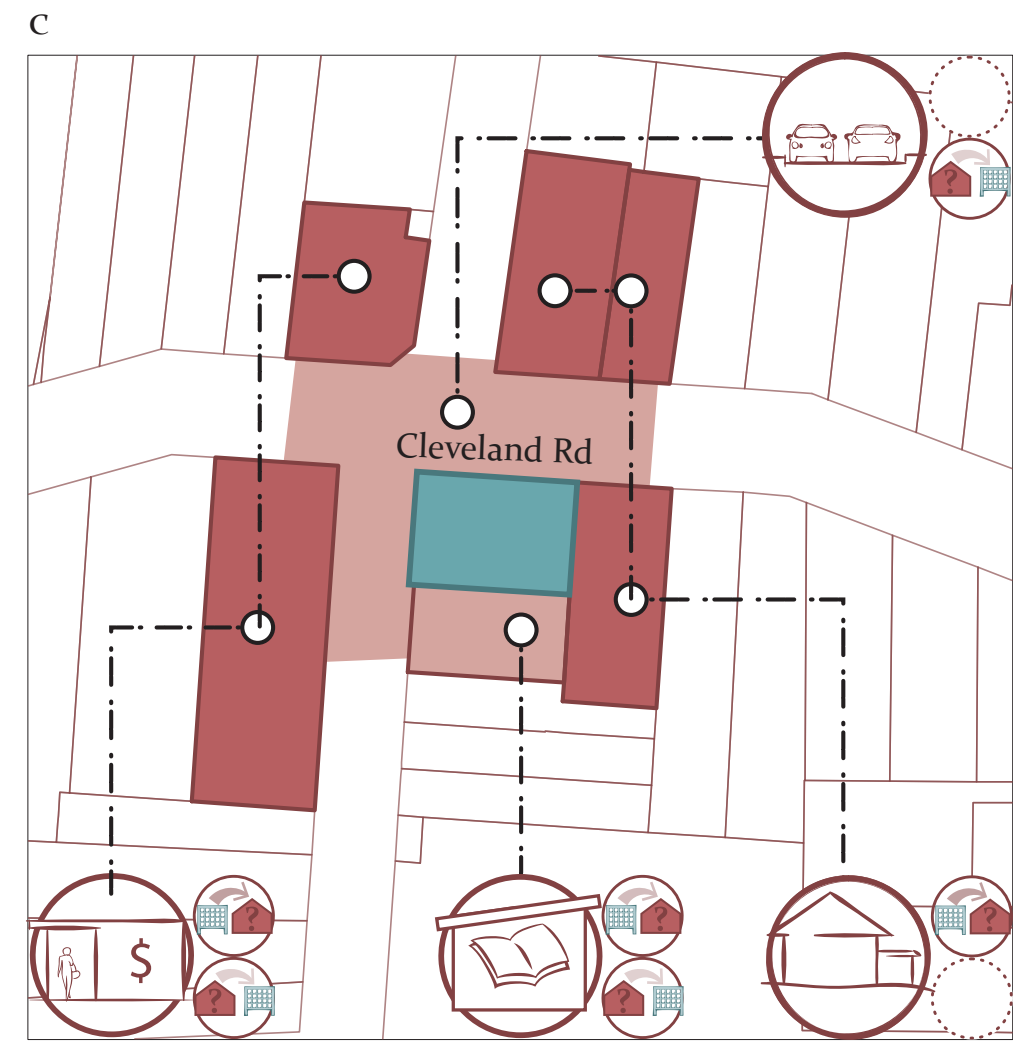

- The control of library thoroughfare will be an important design move.

- Impacts on nearby residential will need to be mitigated, particularly the apartment to the north of the site and the house directly adjacent to the east (although the actual building borders with the library not the area in front).

\section{Land Use Legend}

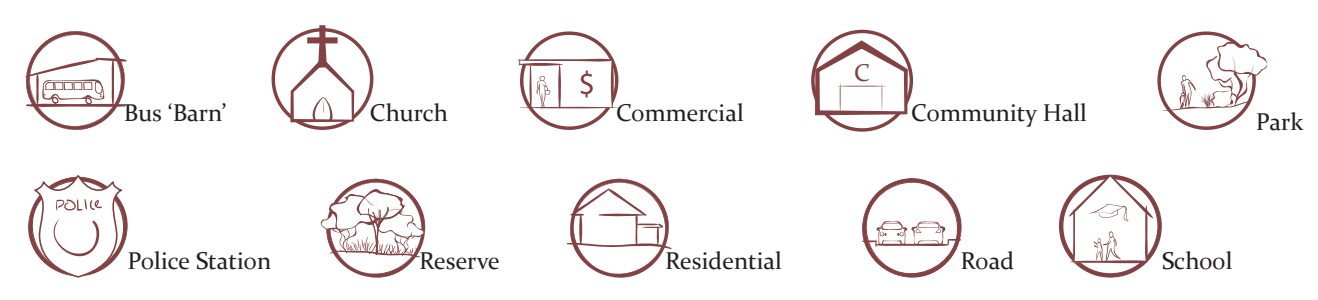

Impacts Legend

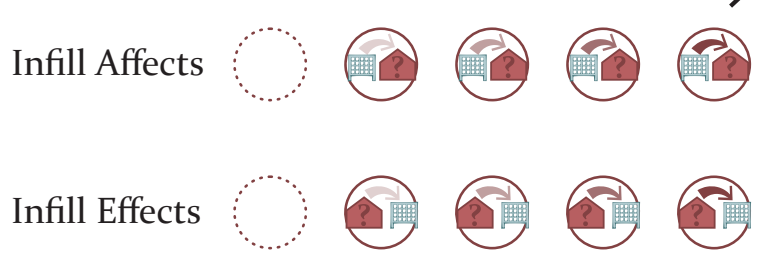


Island Bay

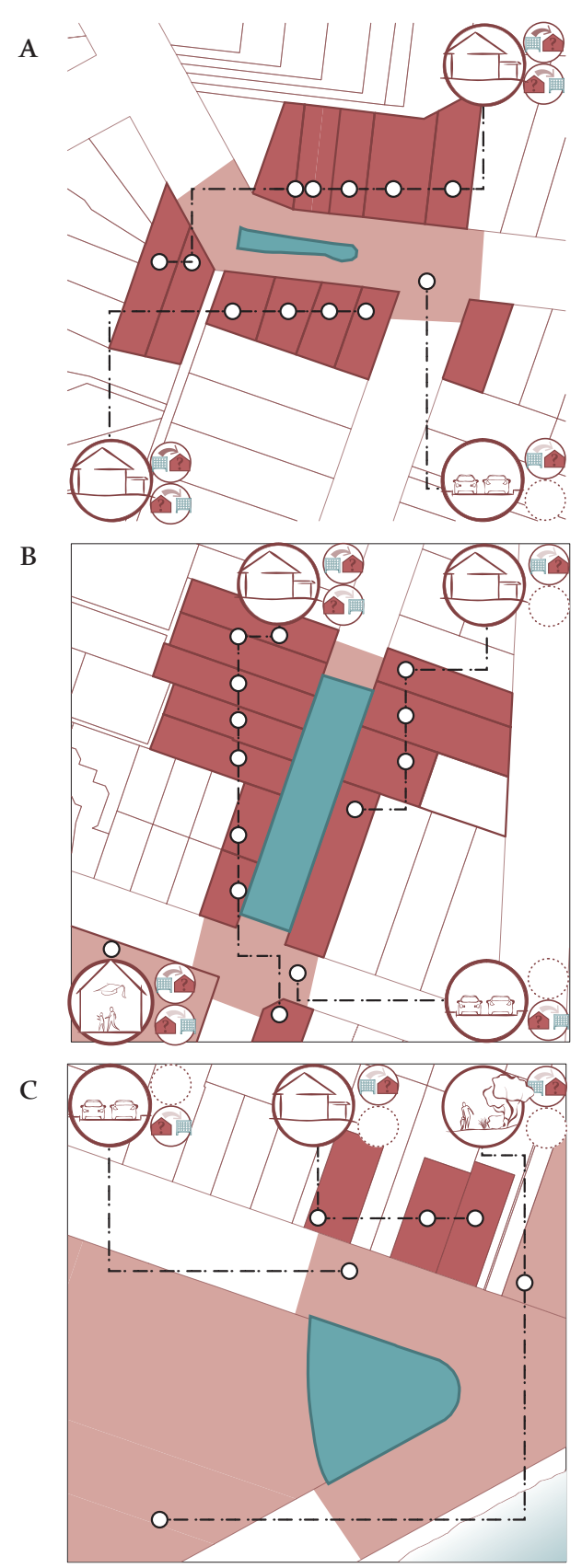

Kilbirnie
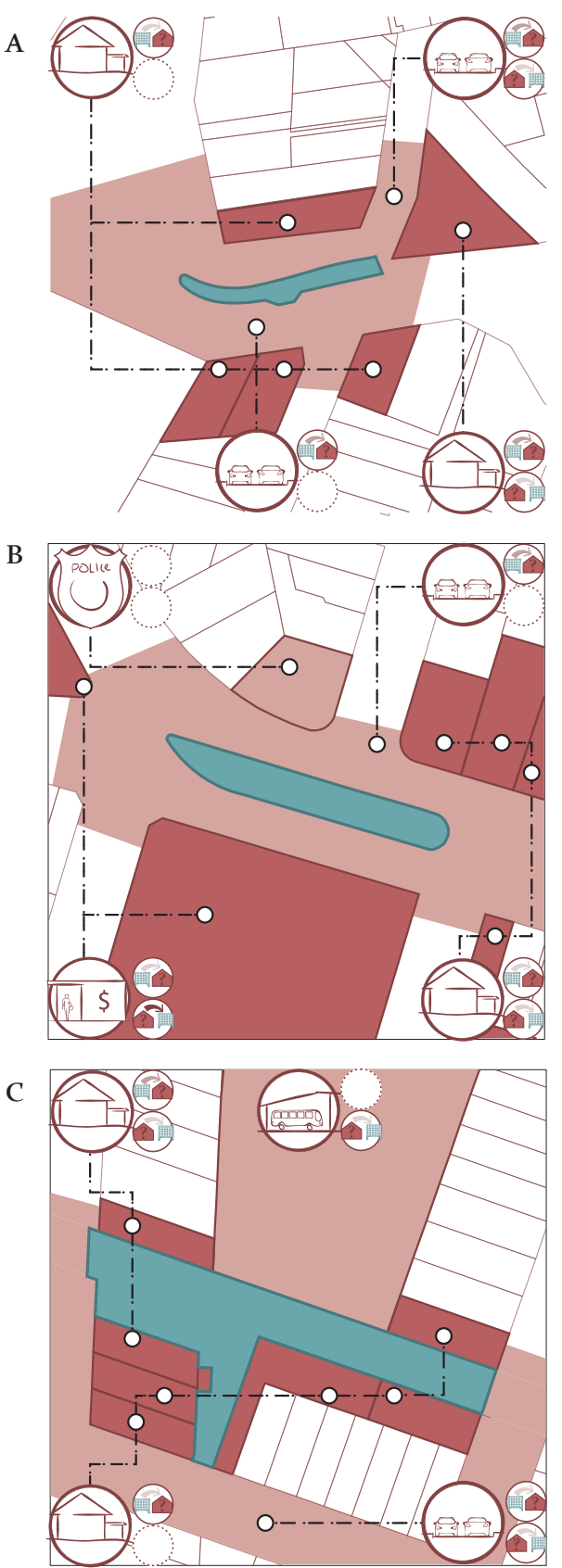

Miramar
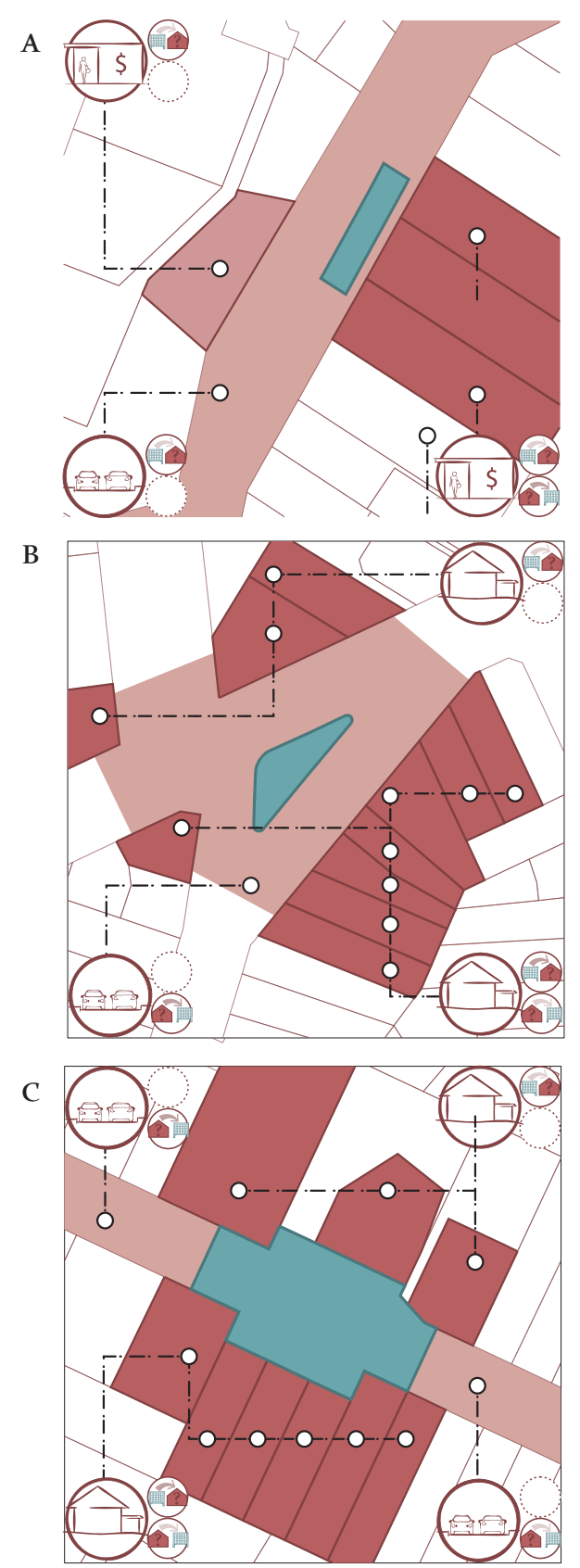

Newtown

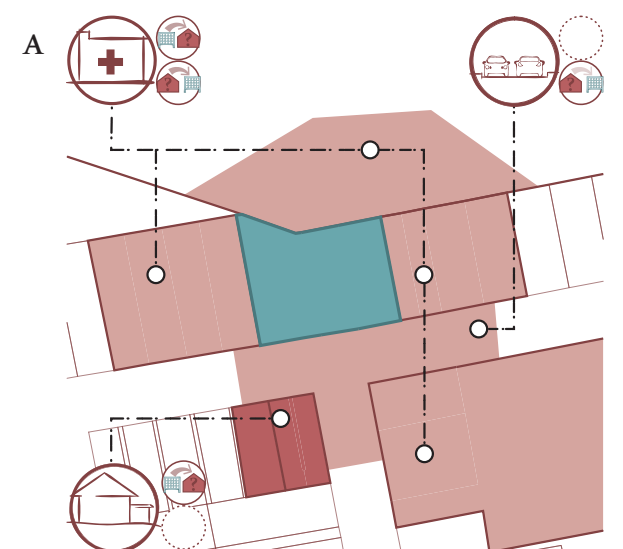

(15)
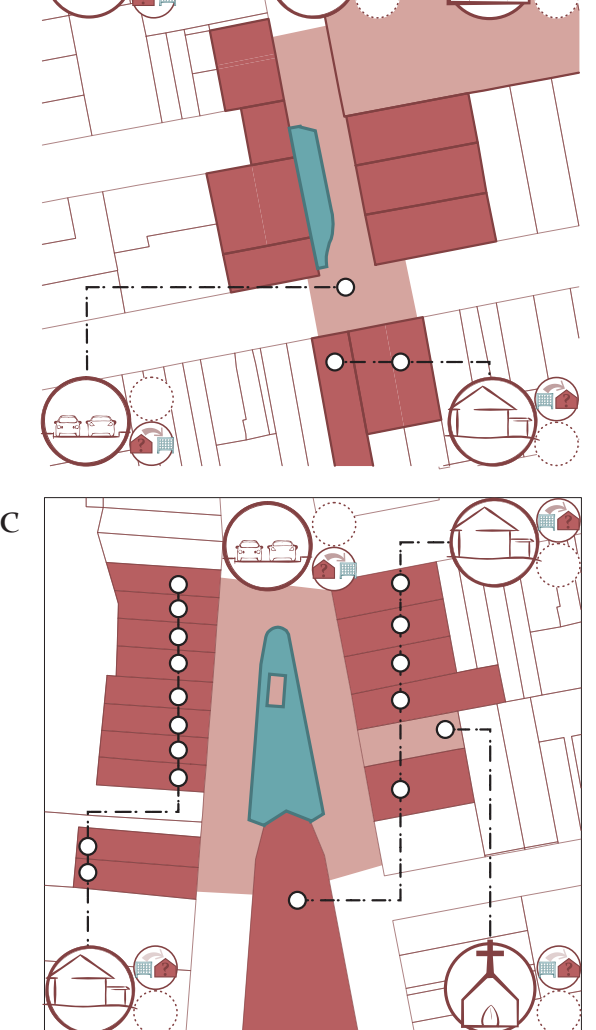

Wadestown
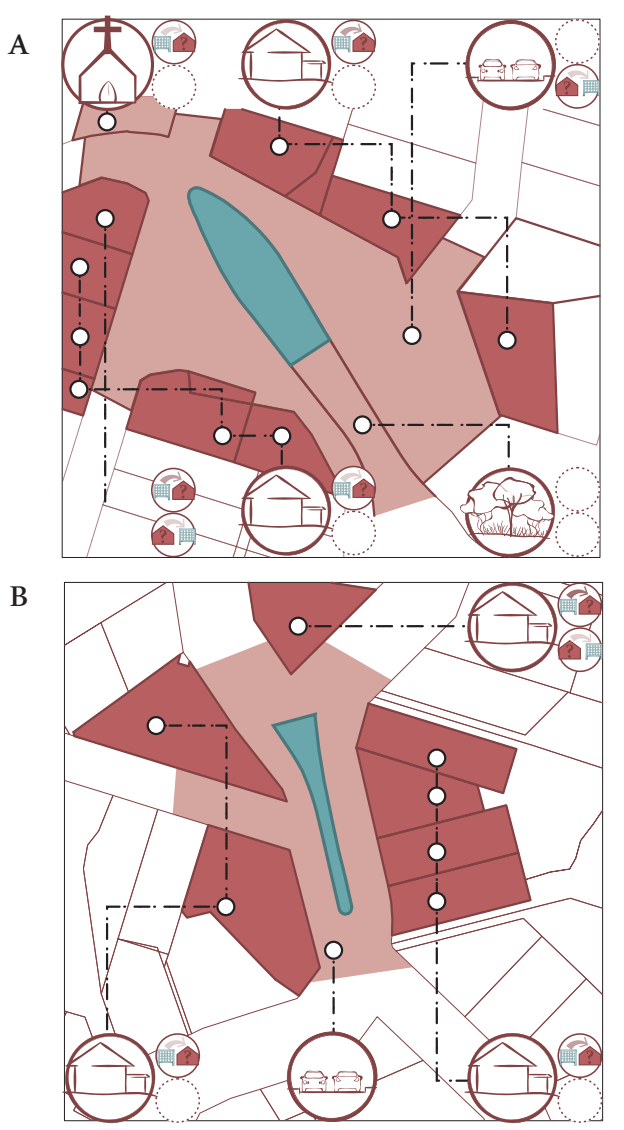

c $308 / / 2 / 59$

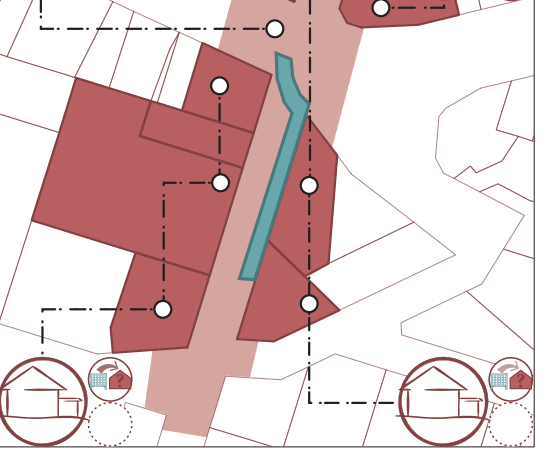




\section{Conclusions}

This site selection process has allowed for the proposal of a network where spaces for the dead have been reintroduced to the suburb's community core. There are many potential sites for these spaces through the city.

The process has led to the selection of these 6 sites across Wellington Cities suburban centres. (Fig 36) Rather than attempt to design all six of these sites, two of them have been selected to be developed in the site design chapter which follows. They are the catalyst project, proposed for construction in Brooklyn in

the next two years, and the final project based in Kilbirnie which would begin in roughly fifteen years time.

While the main contribution of this chapter is the selection of these sites, allowing the design case studies to proceed, it also serves as a test of the first three design principles. Their application here will be critically assessed in the exegesis chapter.
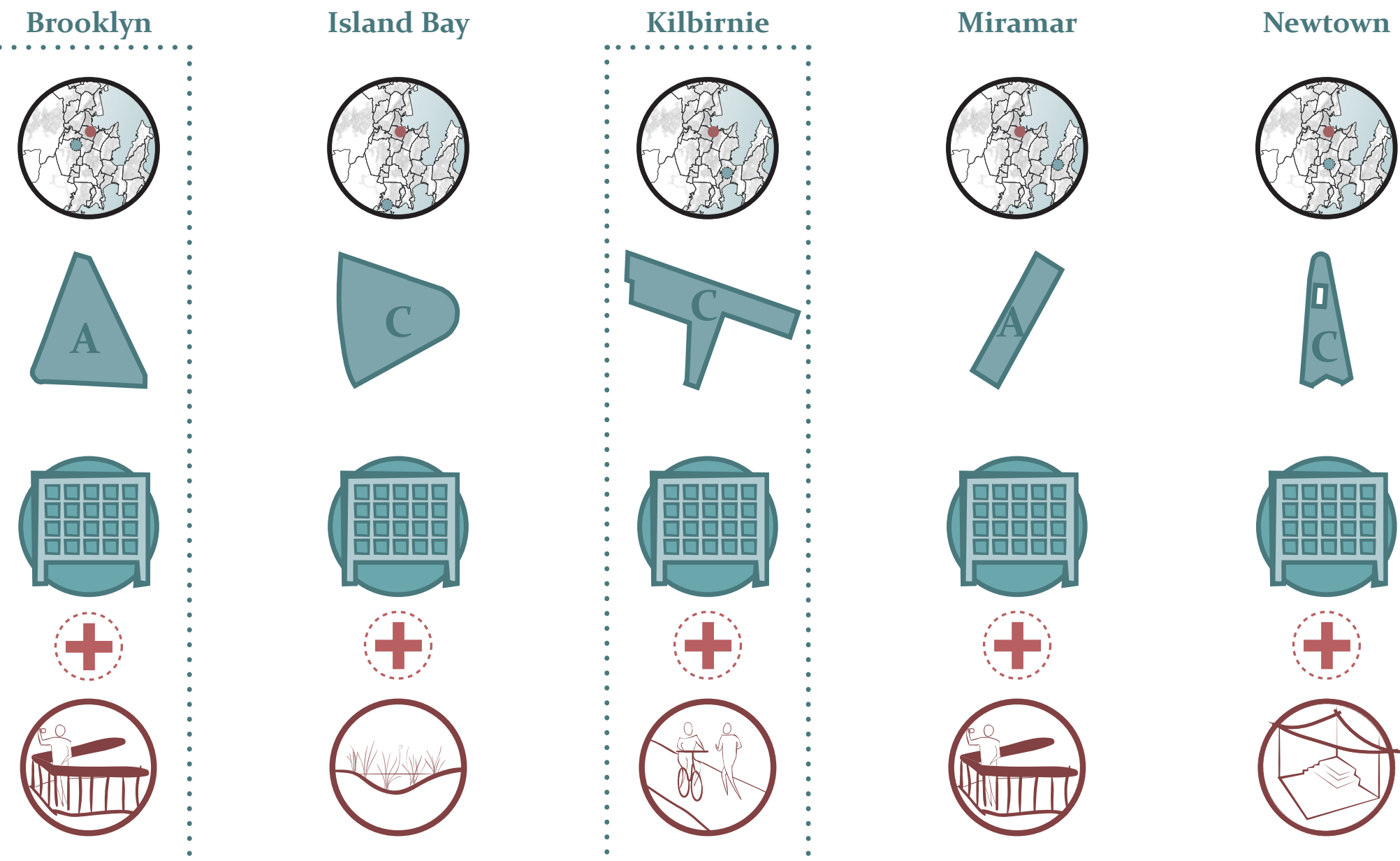

C
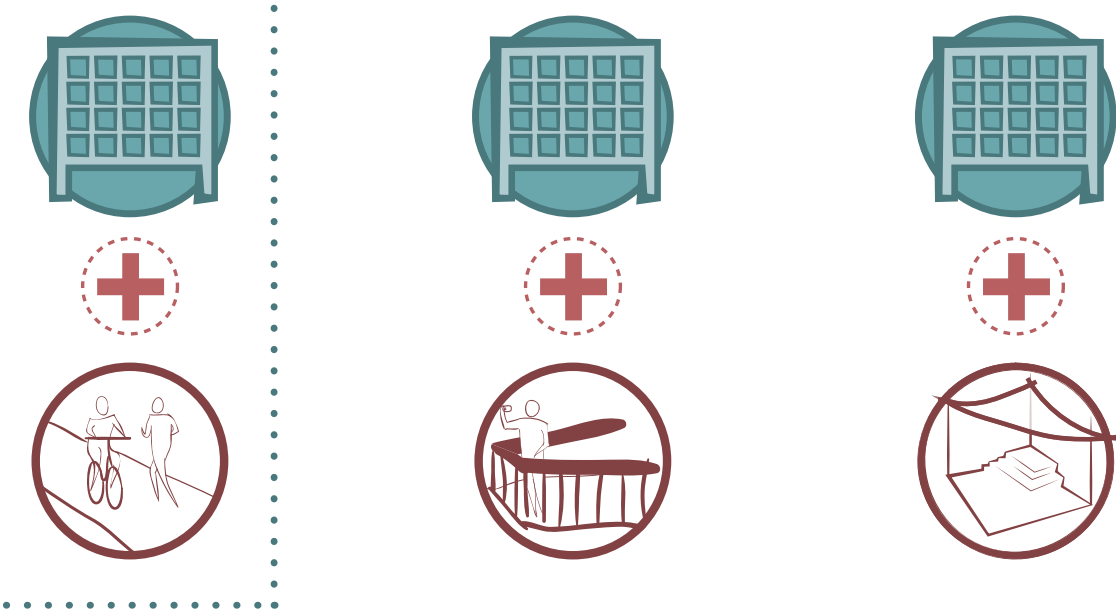

Wadestown
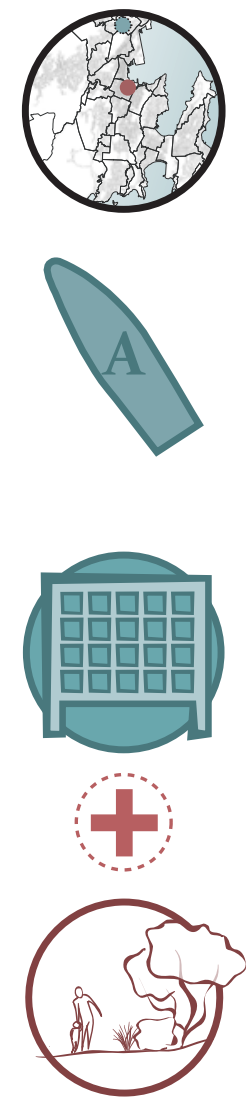


\section{Design}

VI 



\section{Introduction}

n order to find space for the dead in today's suburbs, new ways 1 of organising and designing these spaces must emerge. This chapter seeks to investigate new spatial solutions in the context of two design case studies, the first in Brooklyn acting as a

catalyst to a shift in the conventions for spaces for the dead, and the second in Kilbirnie aligning itself with a proposed suburban intensification scheme.

These designs respond to the location in which they sit, both in terms of the suburb's wider context and the immediate requirements of the specific site. In each design case study these issues are identified. Next the design principles are applied, particularly Layered Intimacy (4) which is the most focused at the site design scale. However the section of Obvious Improvement (3.2) dealing with integrating both the space for the dead and (3.2) dealing with in another and use of benefit to the community, alsocomes in to play, as this integration is not as simple as placing one next to the other.

The results of this design process are represented through a series of renderings and finally through a series of details relating Individualised Interment $(5)$. 



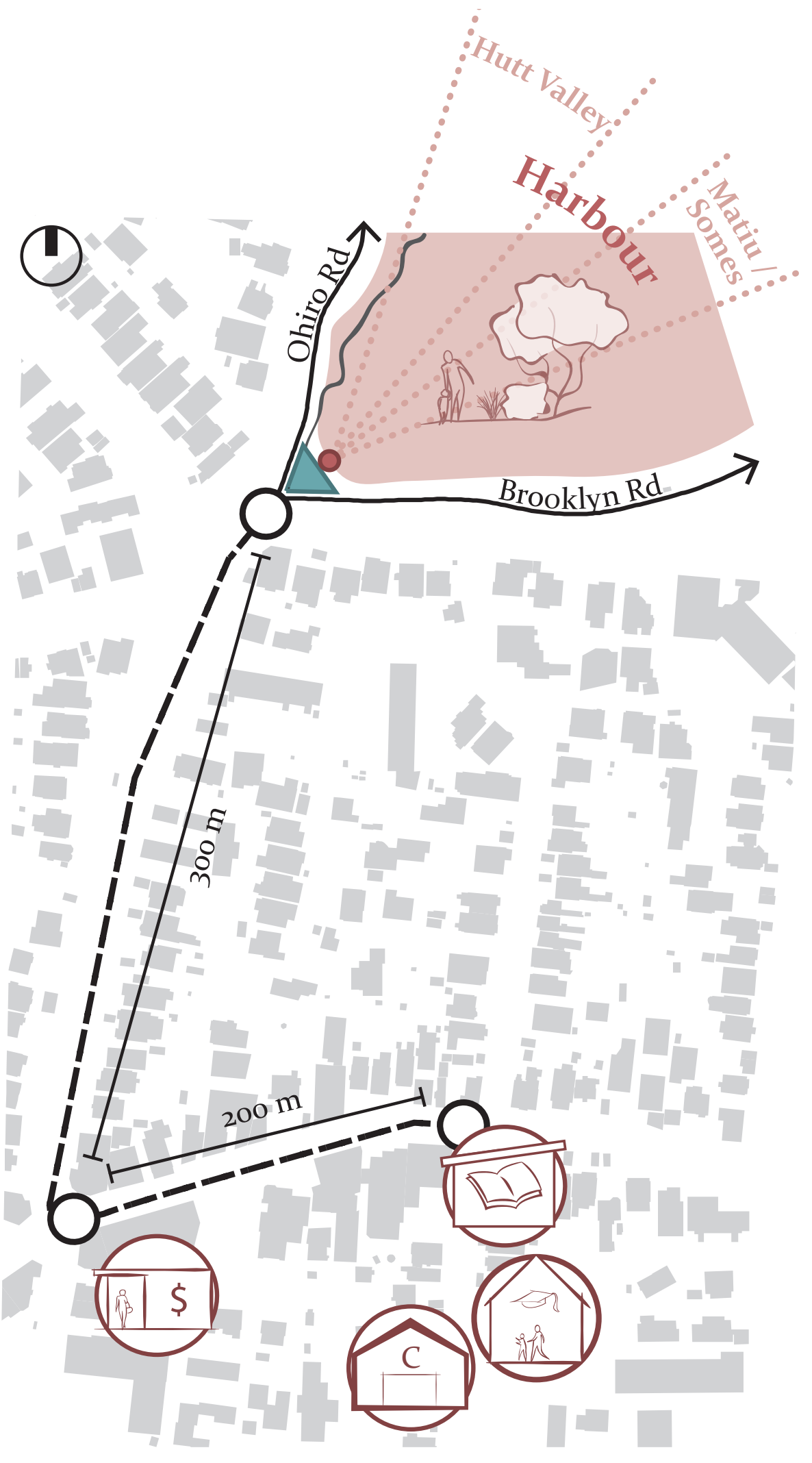

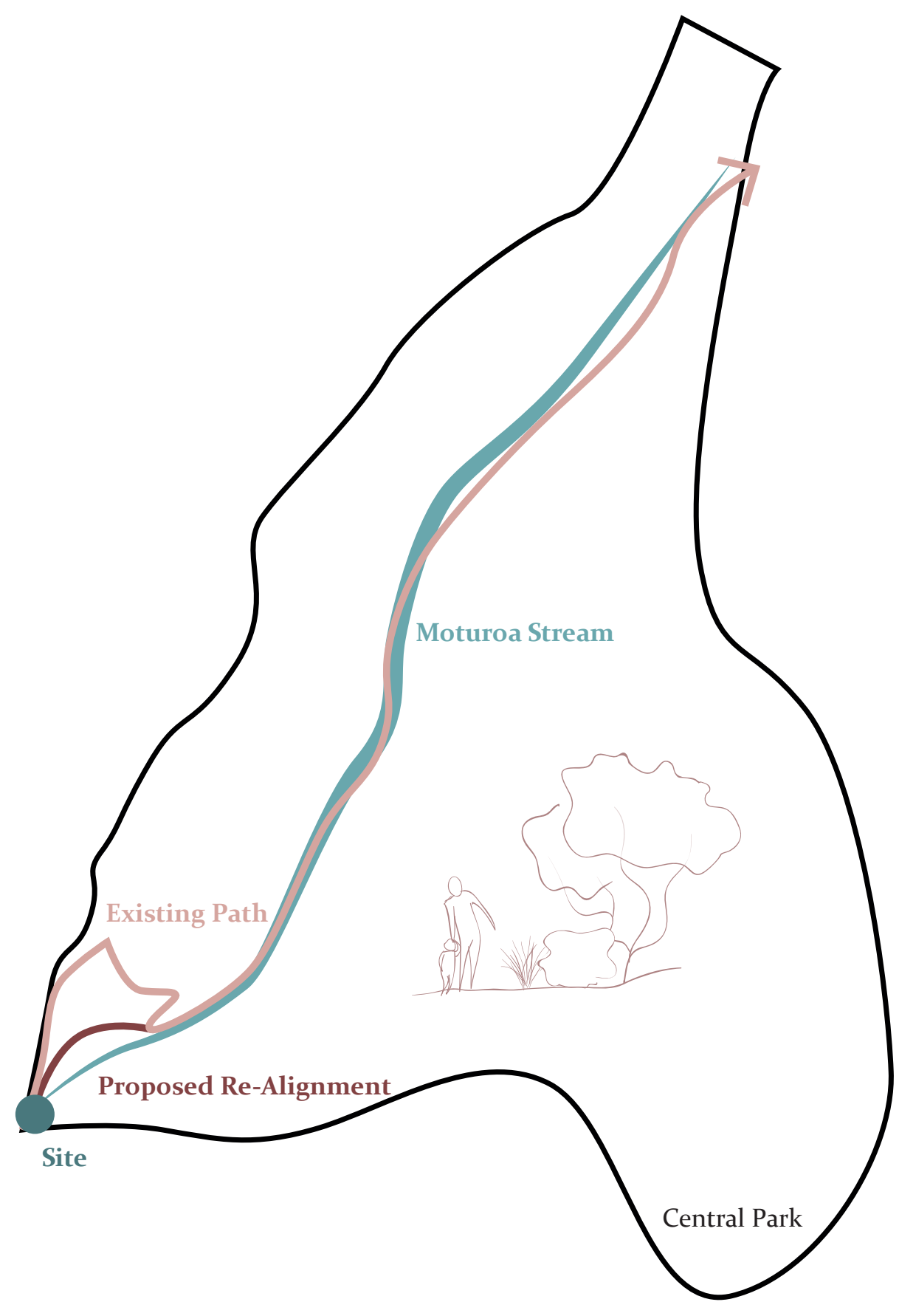

\section{Brooklyn - Catalyst}

The chosen Brooklyn site sits within an interesting context, being both a part of the suburb's suburban core, and the northwestern tip of Central Park. For many pedestrian commuters the quickest way in to the city runs through the site. However the current alignment of the path leaves the Moturoa stream at the stormwater exit where it begins (on the site) and then sharply turns back in towards it later on.

One of the design moves is to realign the path to follow the stream more closely, and to better expose the stormwater exit which feeds the stream, creating a more visible connection between the path and the stream. The site will effectively become the entrance to this path, and as such an entrance to Central Park. 


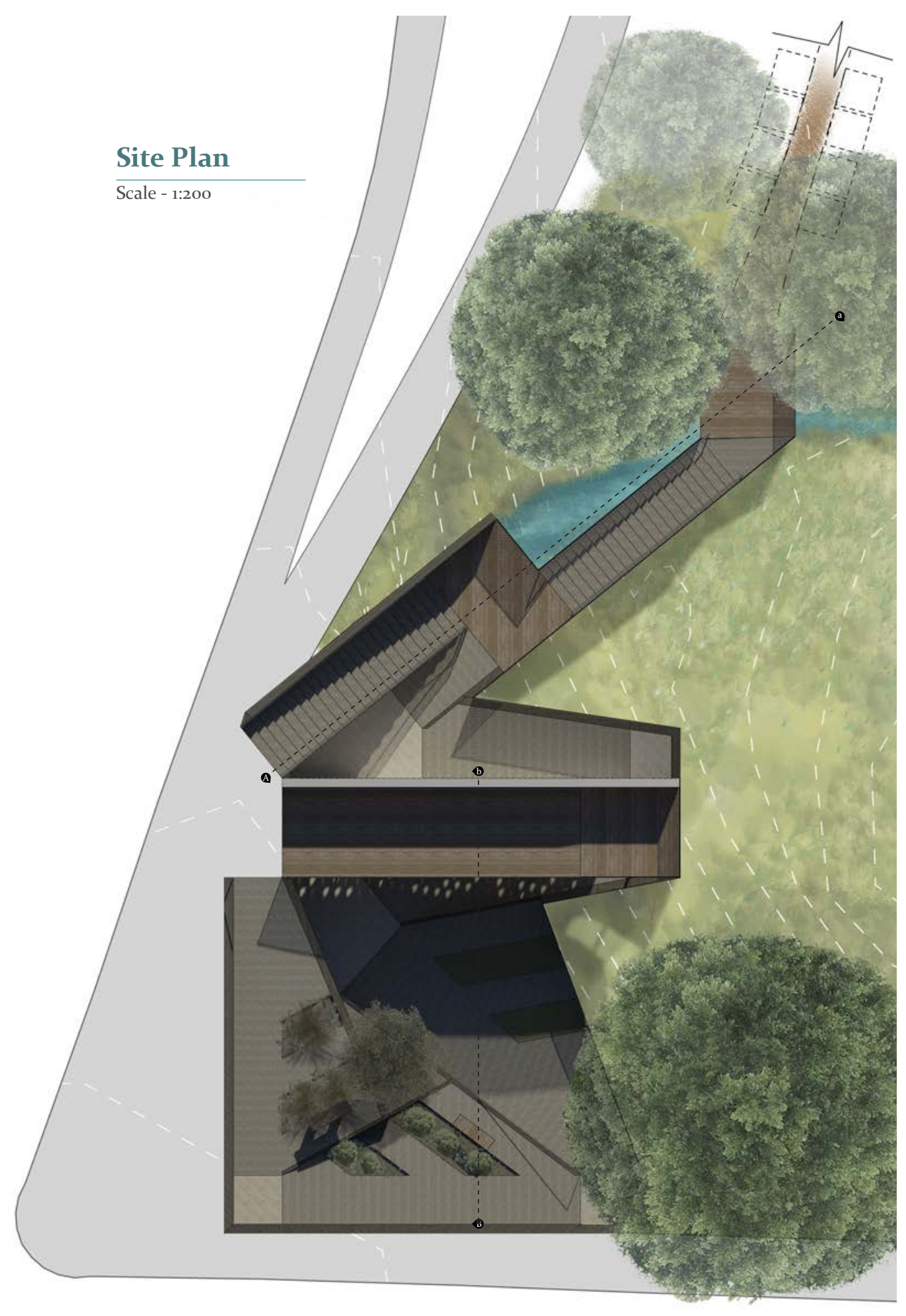

Figure 39. Brooklyn Site Plan
Application of Layered Intimacy Principle

The layered space required to create an intimate space has been implemented here through the four access ramps which slope down and through the

columbarium. The spaces are made distinctive from

one another using planting (and in the columbarium

space, the canopy of the viewing platform). The

(n)

to provide a tactile indicator.
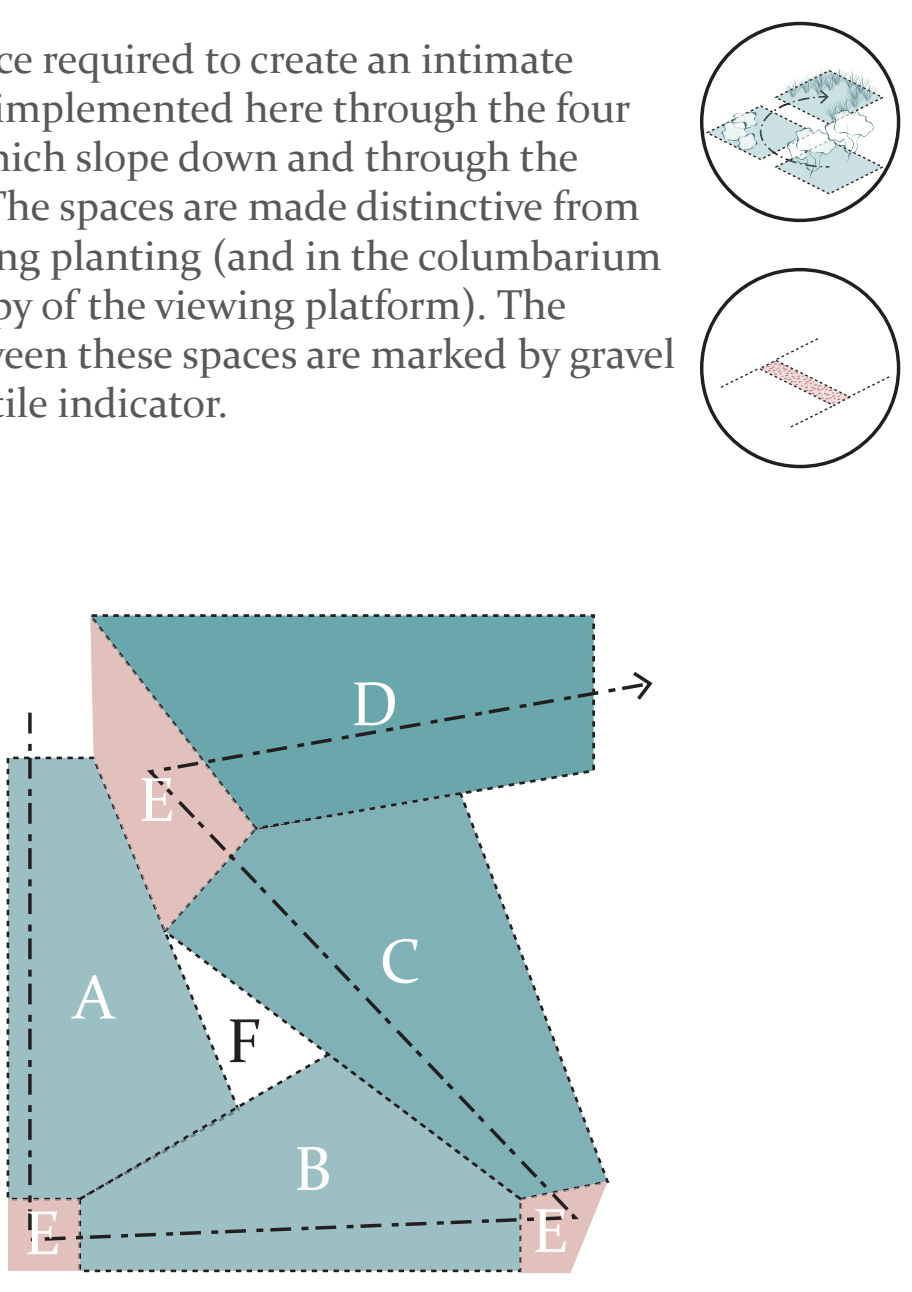

C
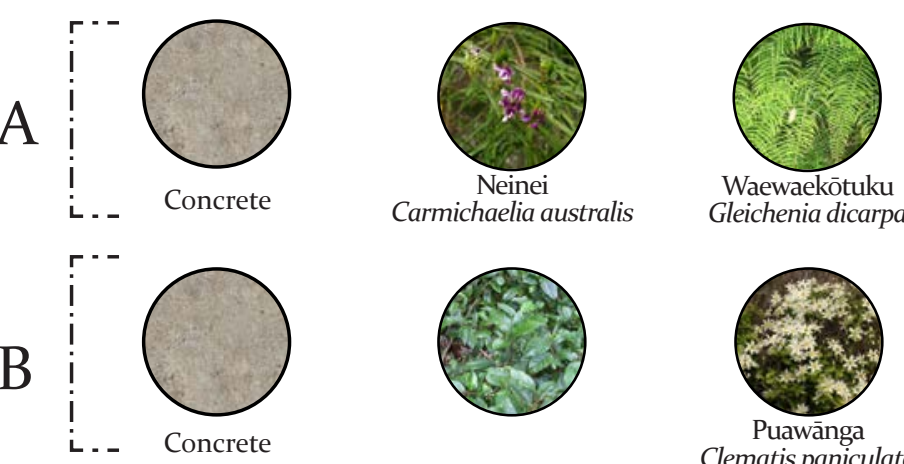

$\vdots$
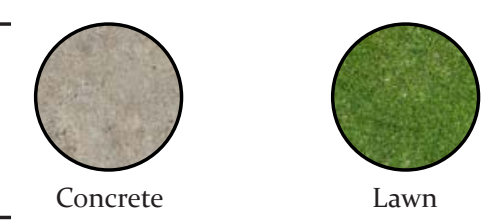

$\mathrm{D}$

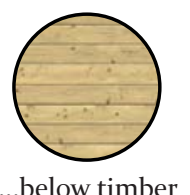

i.. Concrete...

...below timber

E

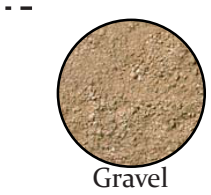

F
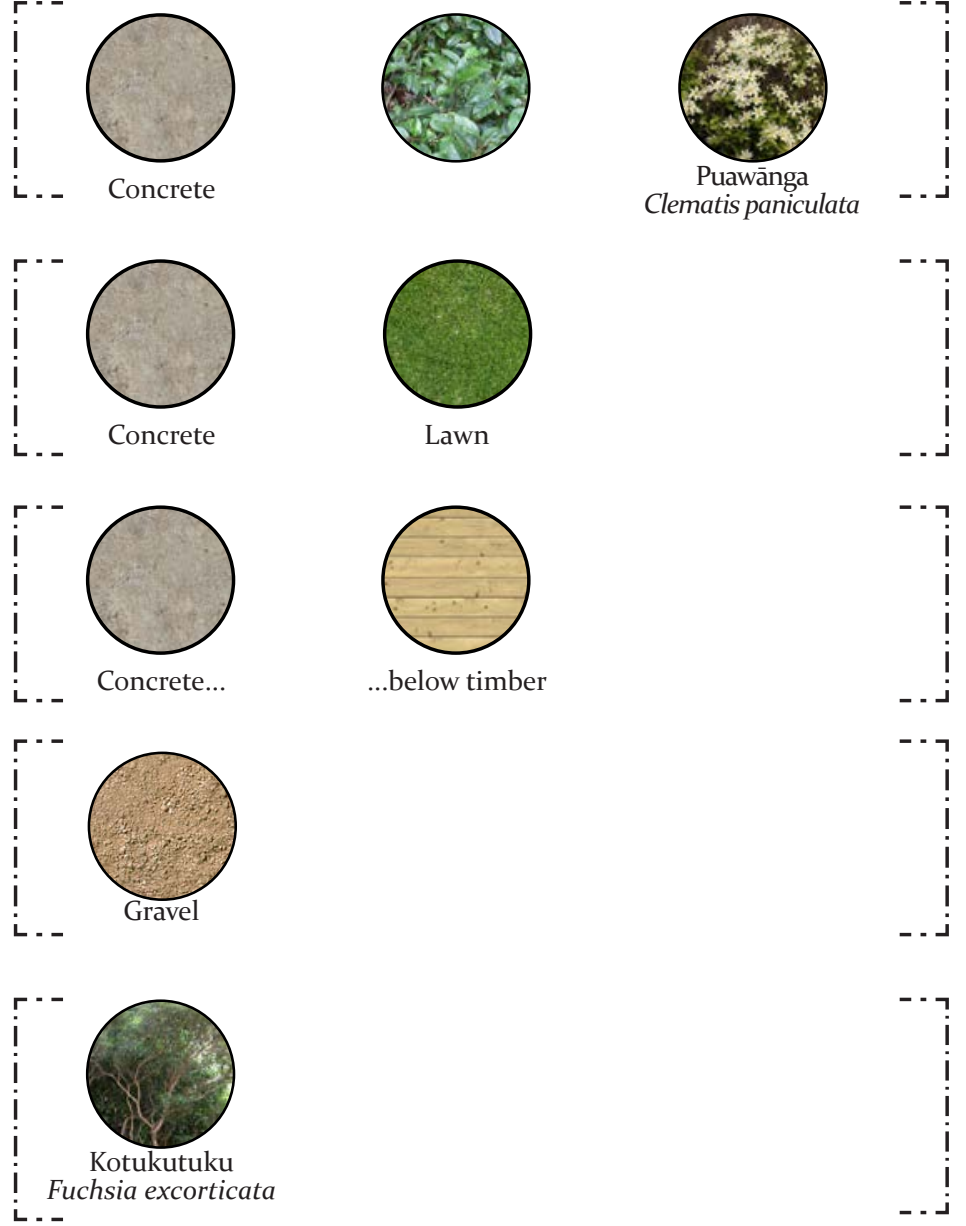

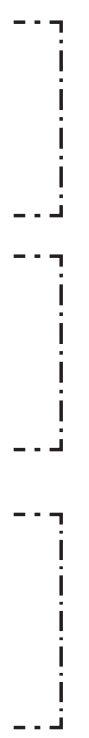

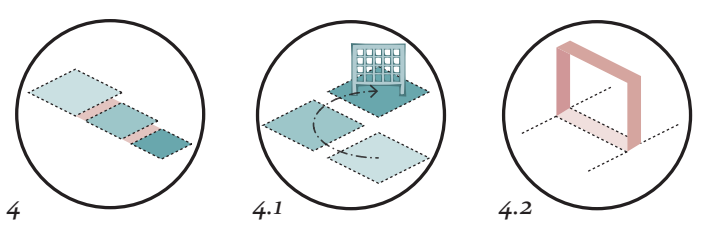

Figure 40. Planting and Surface Palette.

Source: Wellington, Greater. "Wellington Regional Native Plant Guide." Wellington Greater Wellington, 2010. Print. 
Scale - 1:100

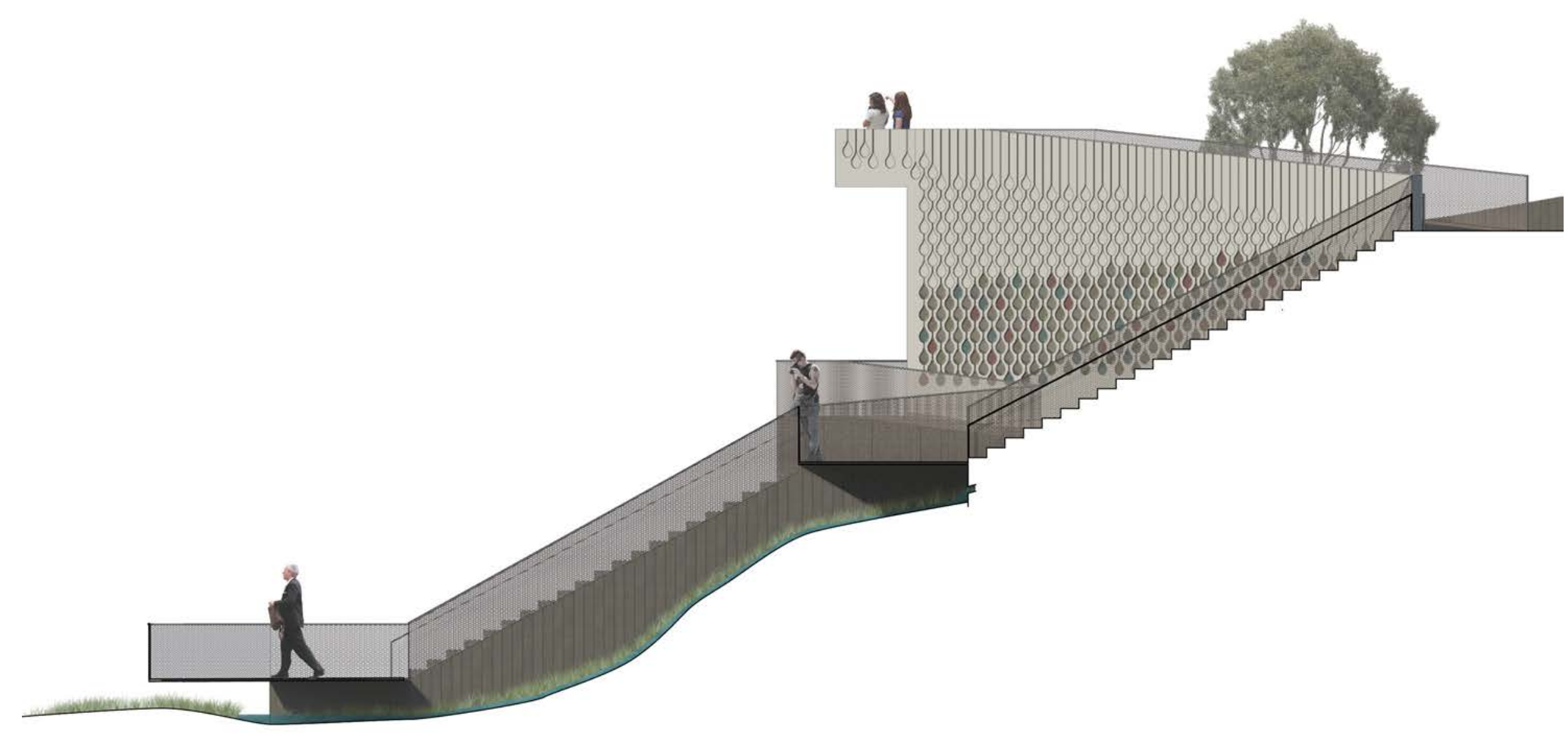

Application of Obvious Improvement Principle

Adding value through another land use, while initiated during the site selection process, must also be executed at the site scale.

While it may be tempting to simply place the two land uses next to one another, this serves little purpose. An advantage to actually integrating the two land uses within the site is that the purpose of the space for the dead becomes more subtle.

Here we can see that the columbarium has become a support for the viewing platform, while the platform has become a canopy for the columbarium space. This means that those simply passing through as they walk in to town through the park will not have the death aspect of the site 'forced' upon them, while the wall still provides enough intrigue that anyone interested can still choose to investigate its true purpose.

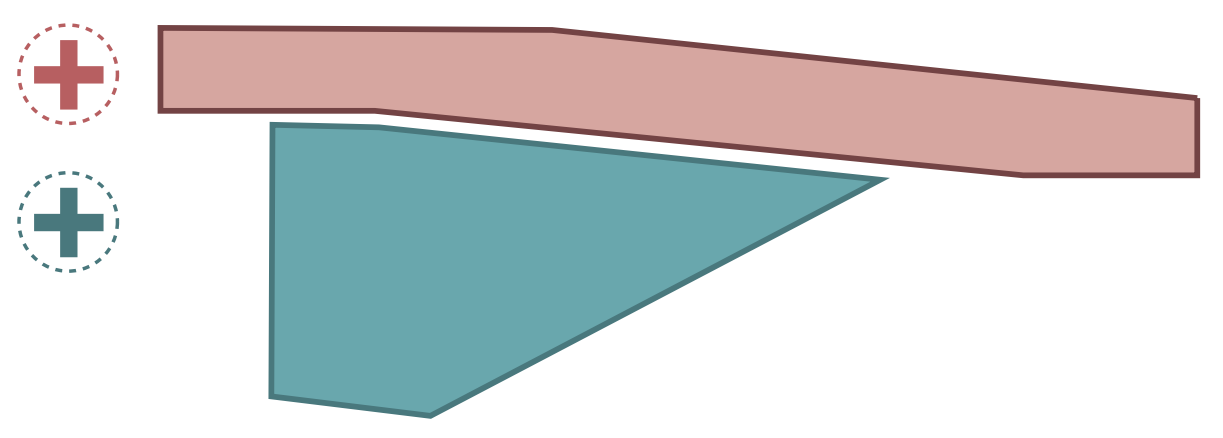




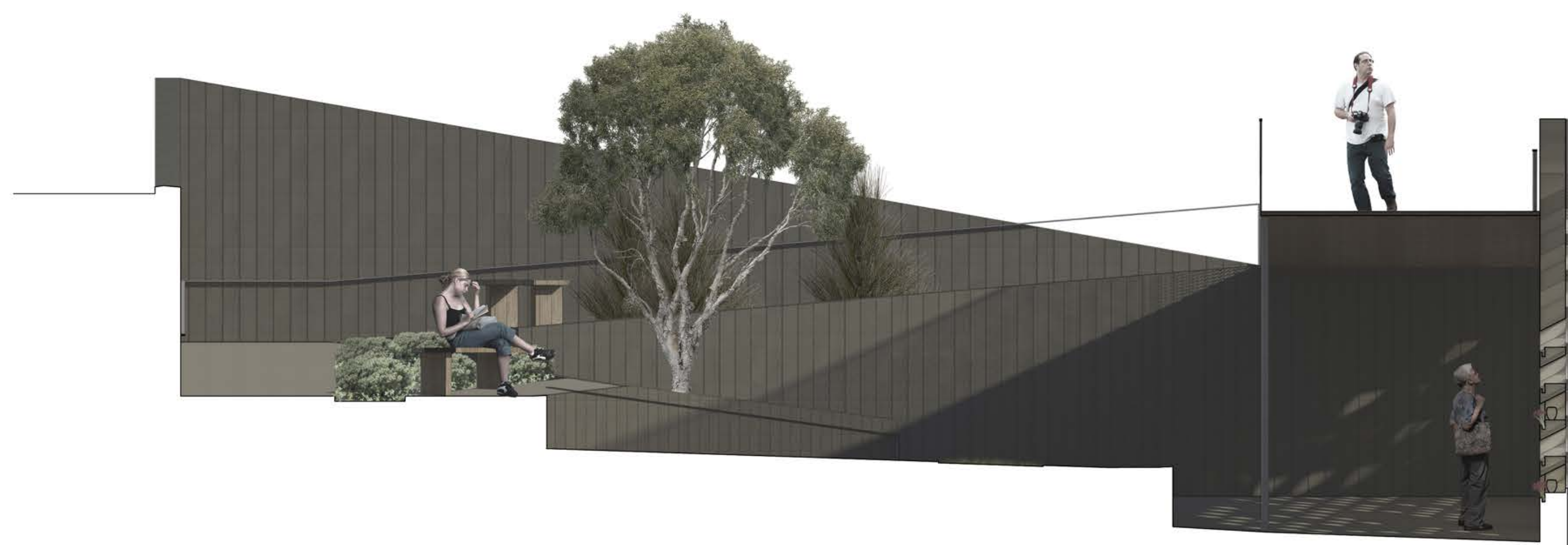




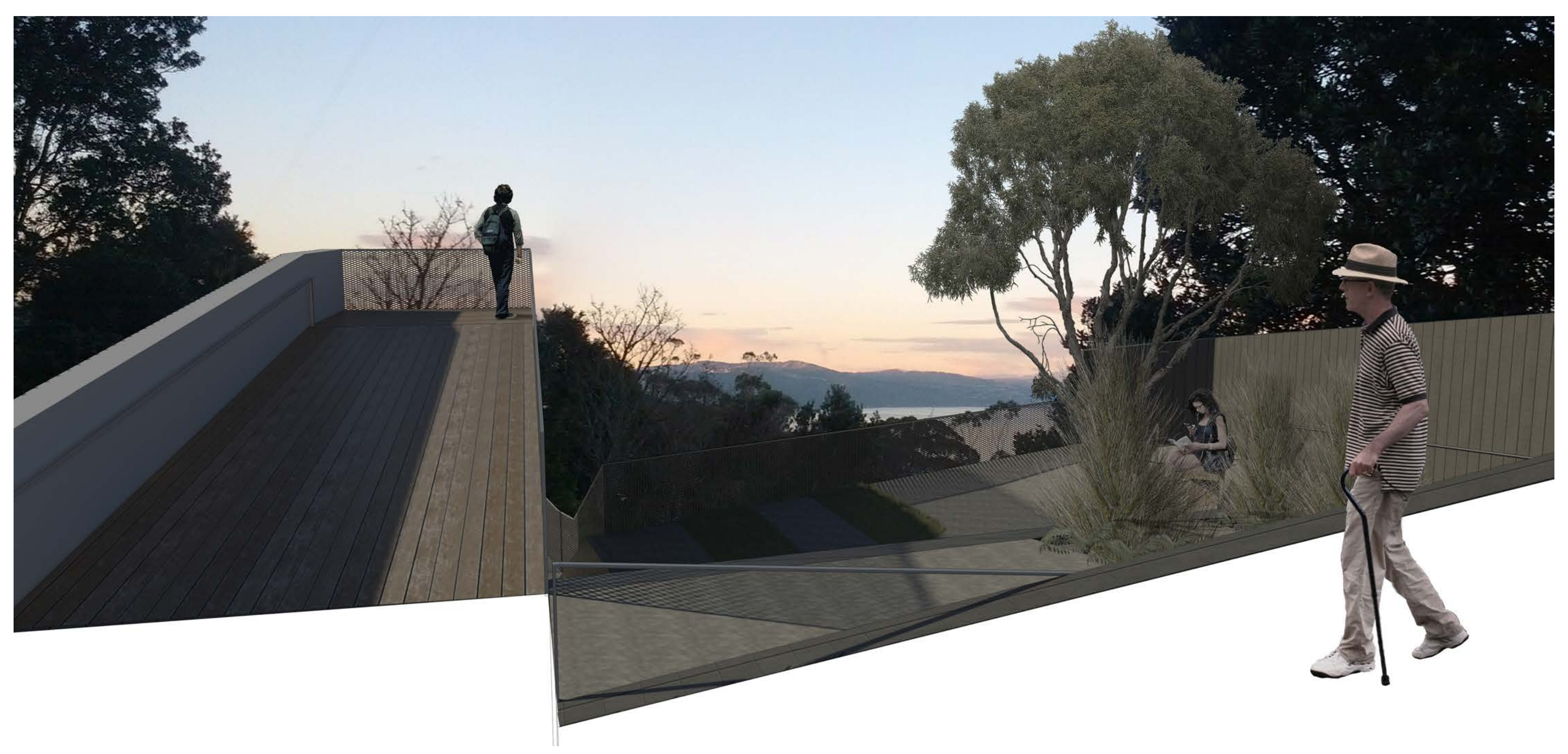




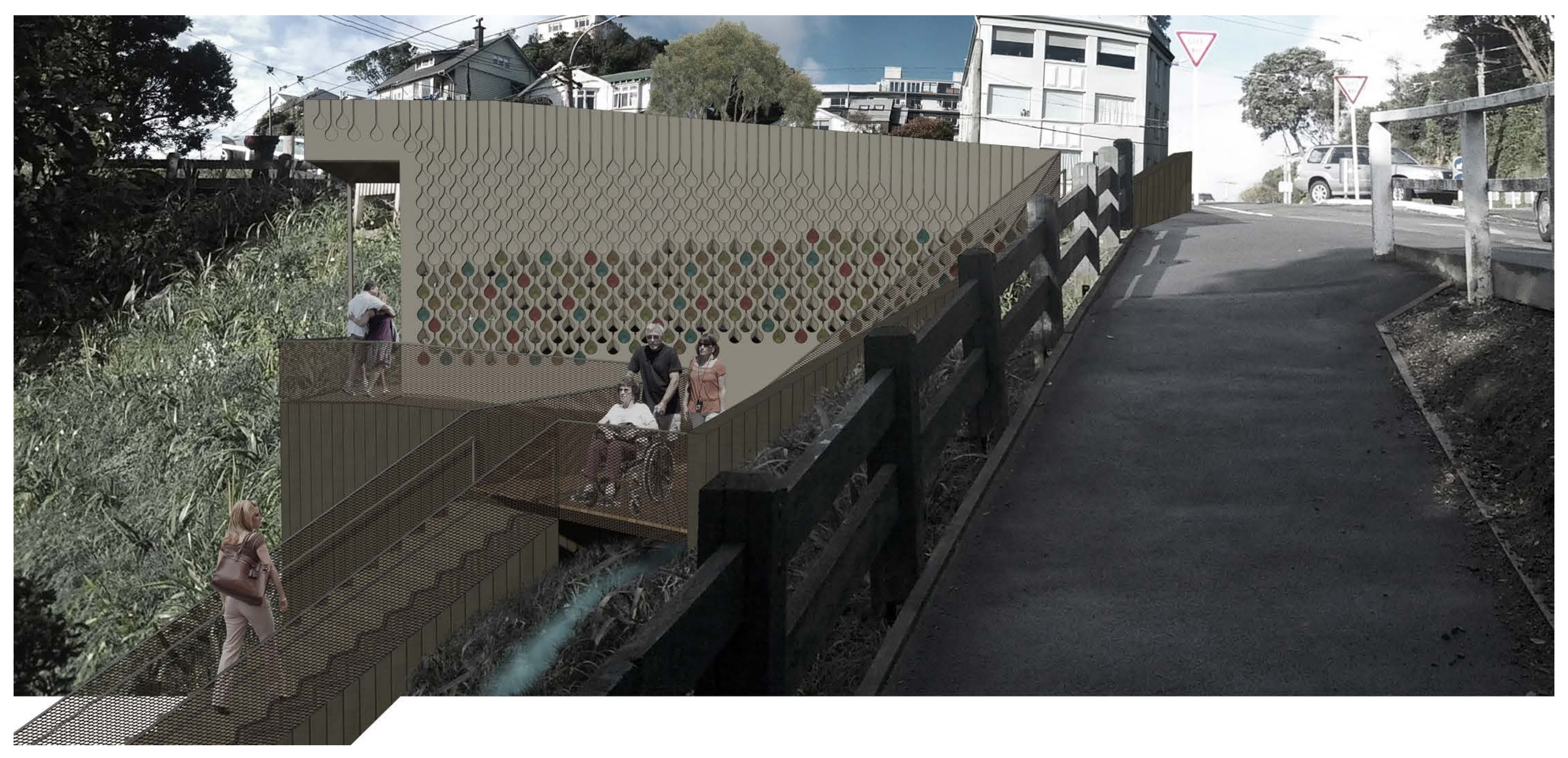




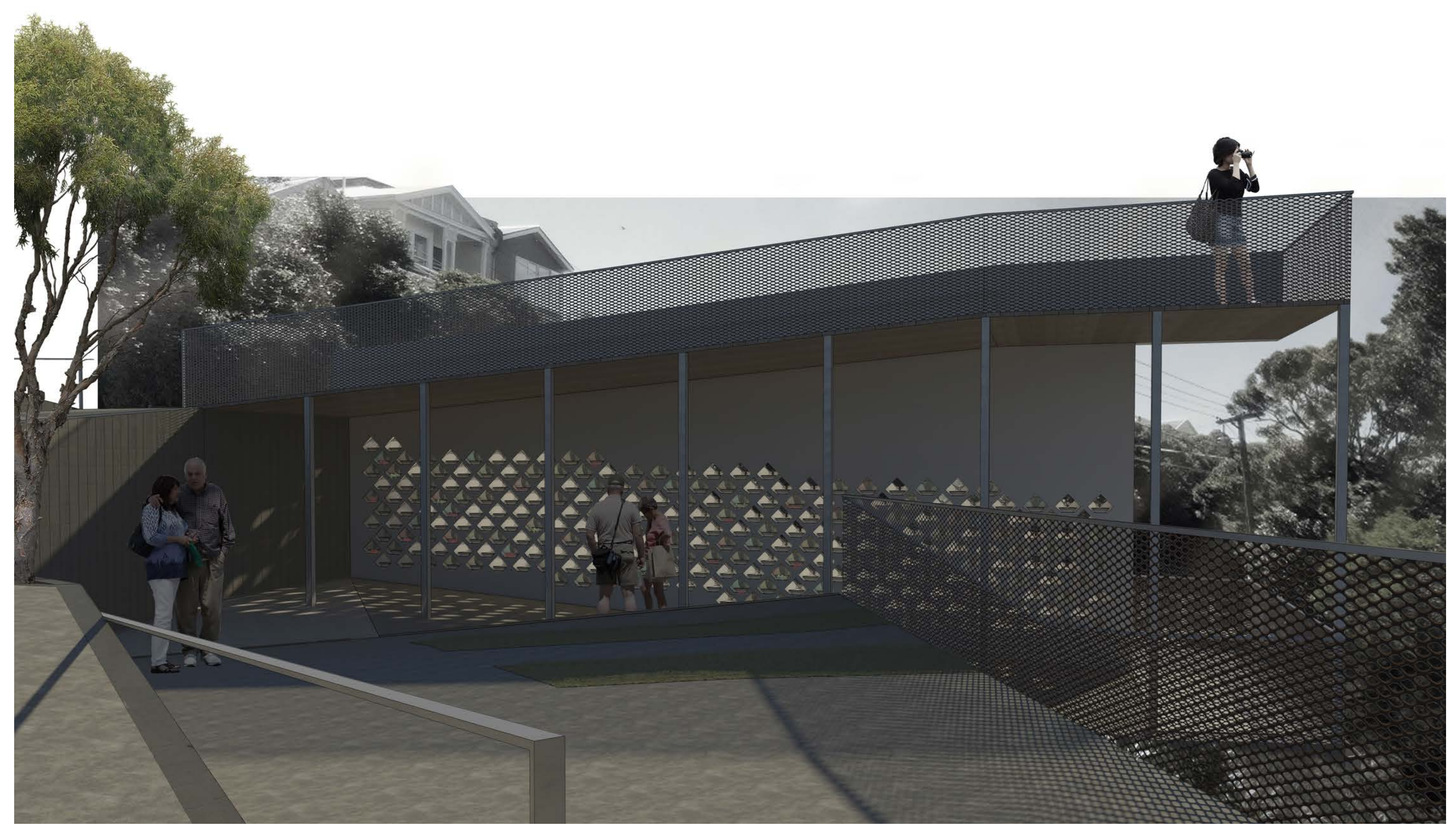





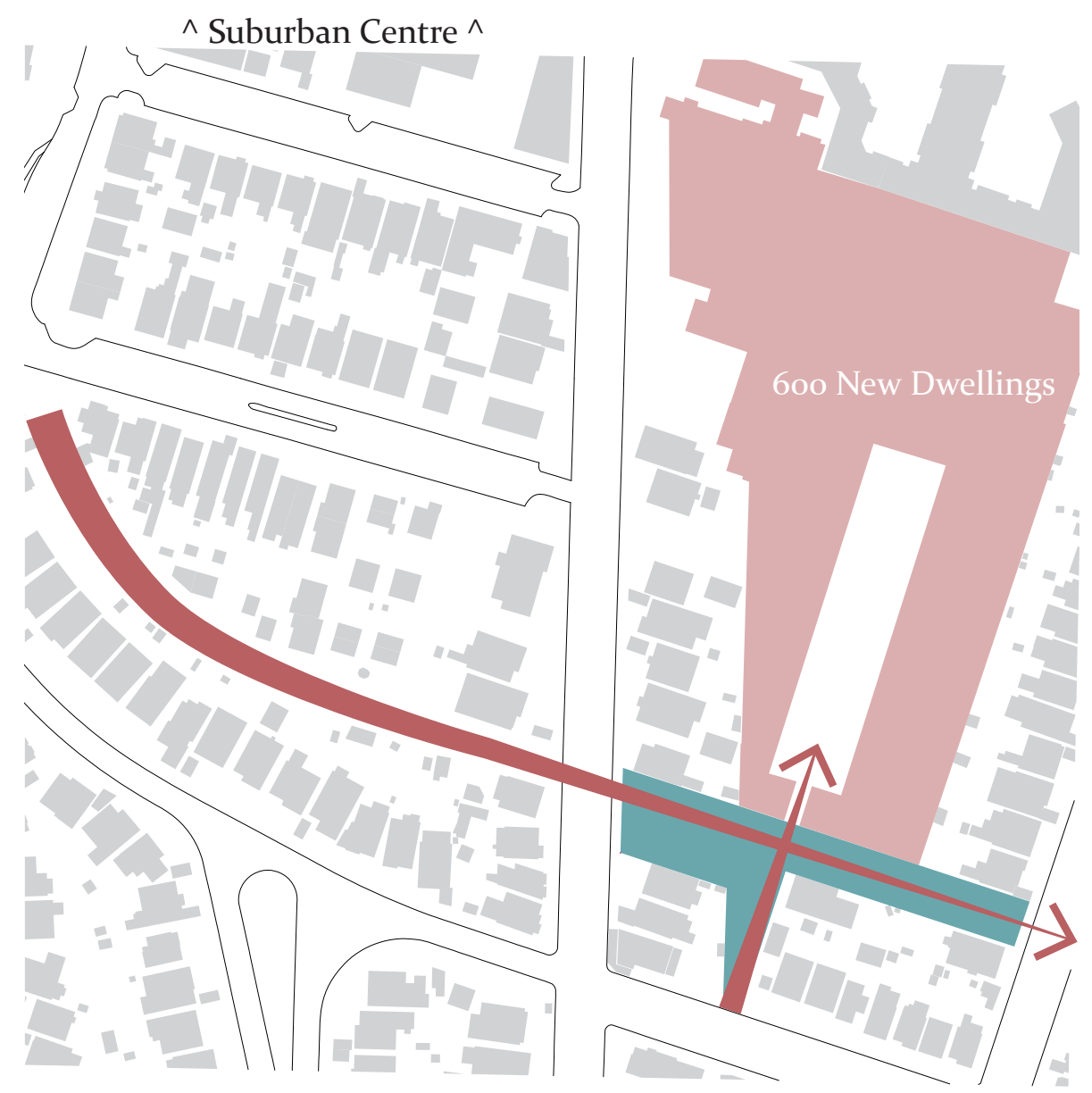

\section{Kilbirnie - Development Alignment}

The Kilbirnie Town Centre plan published by the Wellington City council proposes a medium density housing development on the site of the current 'bus barn' which will provide 600 dwellings for the suburbs intensification by 2030. In addition to this a community walkway is proposed running along the existing services easement from the development to the suburban centre.

The site of the columbarium is within this walkway, aligned with the bus barn development. The design seeks to accommodate both the walkway and an entrance space to the development, while also integrating a columbarium, providing the option of local interment.

The design was required to leave the centre of the walkway without major intervention in order to allow access to the

infrastructure below. A series of programmatic strips ranging from sculptural plinths to picnic tables (and of course the columbarium space itself) were established in order to foster community interest in the walkway beyond its pedestrian transport role.
Application of Obvious Improvement Principle

The integration of the two land uses is, in this case, slightly less literal than in Brooklyn. The columbarium serves as a circulation alignment aide for the walkway and entrance, creating a node of sorts at the intersection of the two paths.

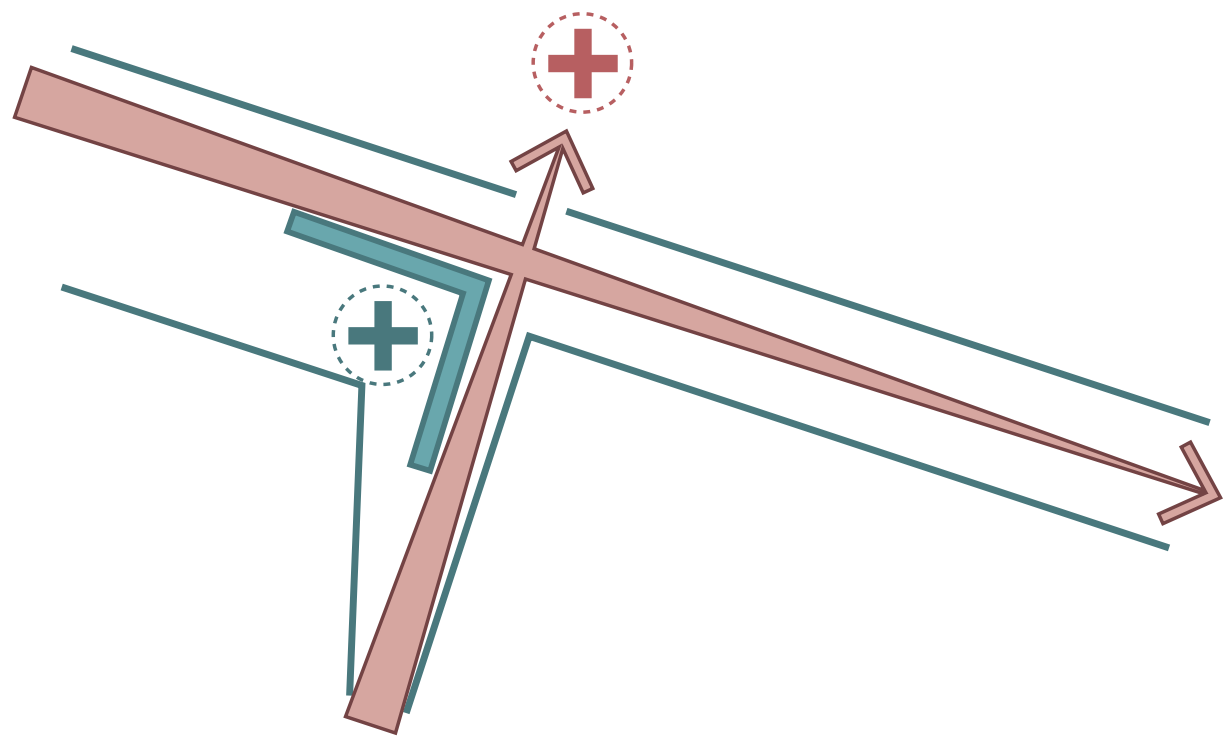


Site Plan

Scale - 1:500

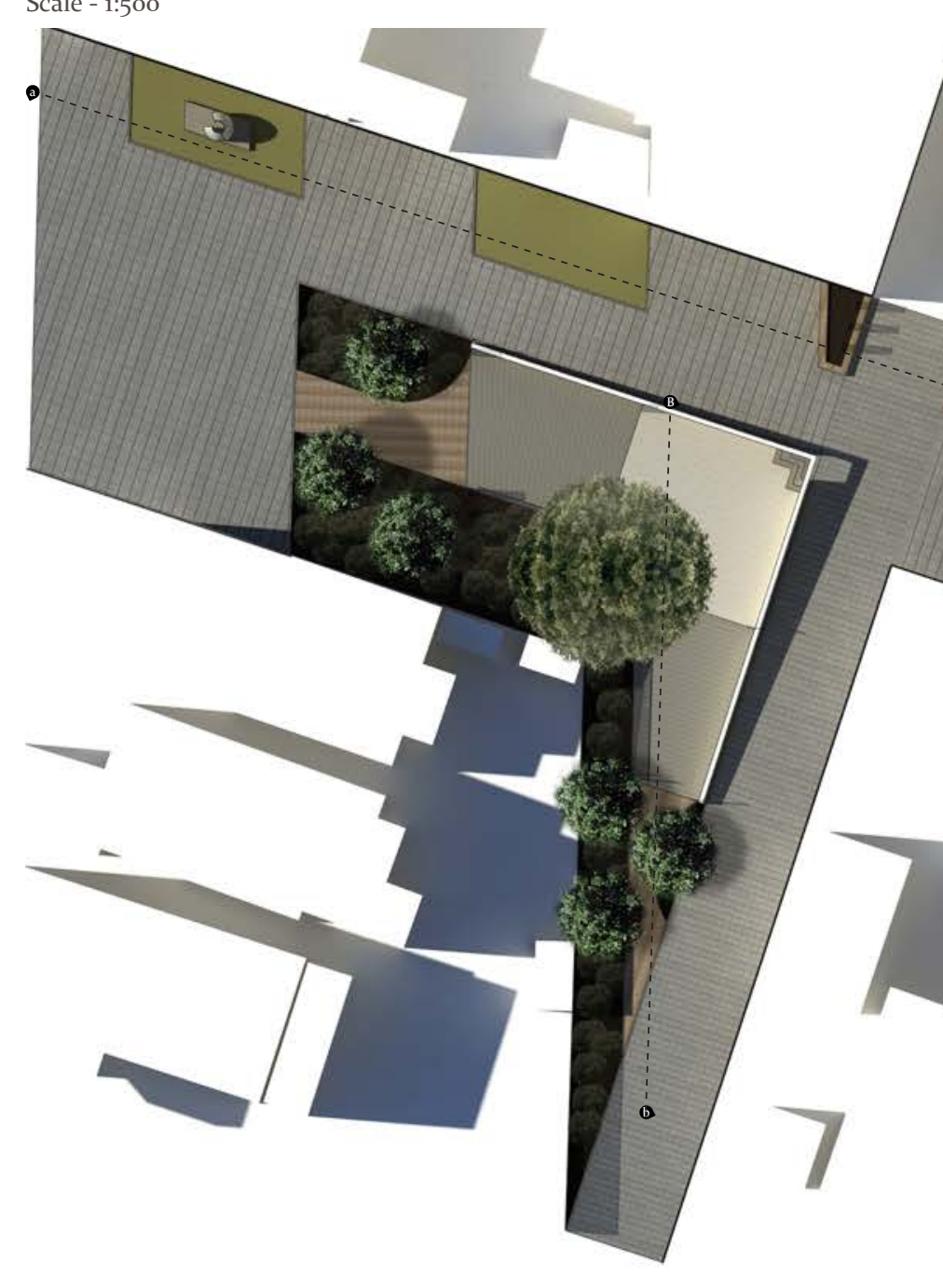

Section Aa

Scale - 1:200

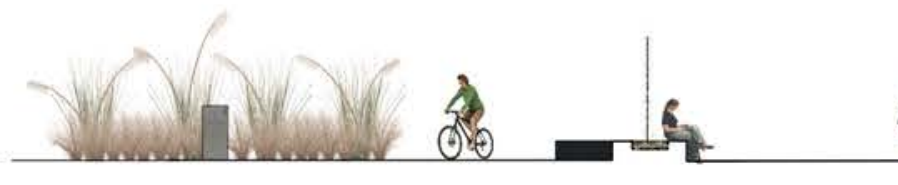

Planting Plan

Scale - 1:1000

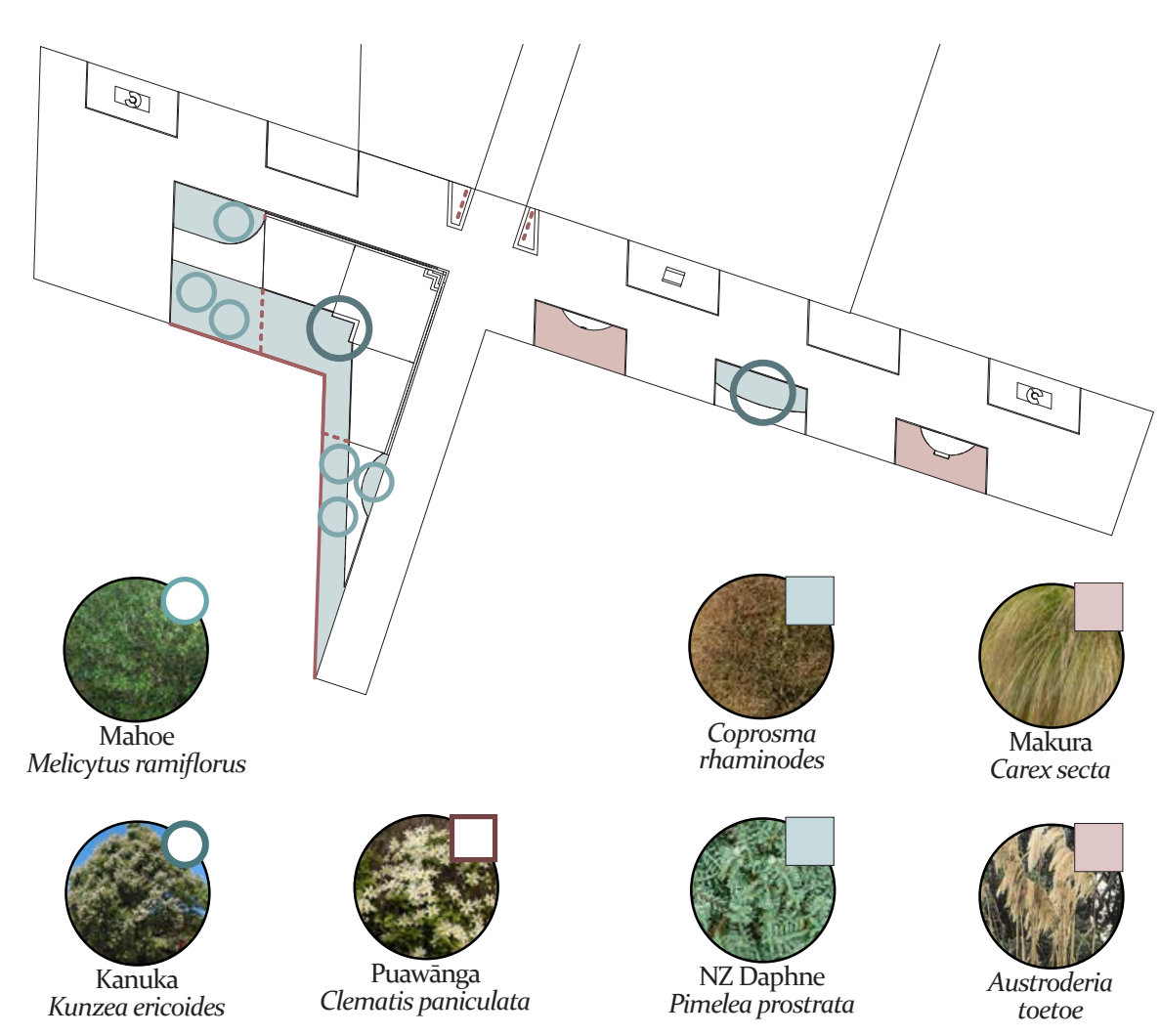

Application of Infill Impacts Principle

The planting here is used to create a 'screen' (along with the perforated steel fences) between the columbarium space and

the residential, mitigating the potential impacts which were

identified within the site selection chapter.

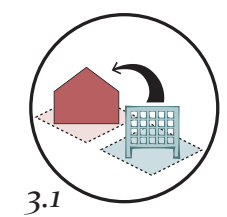


Section Bb

Scale - 1.100
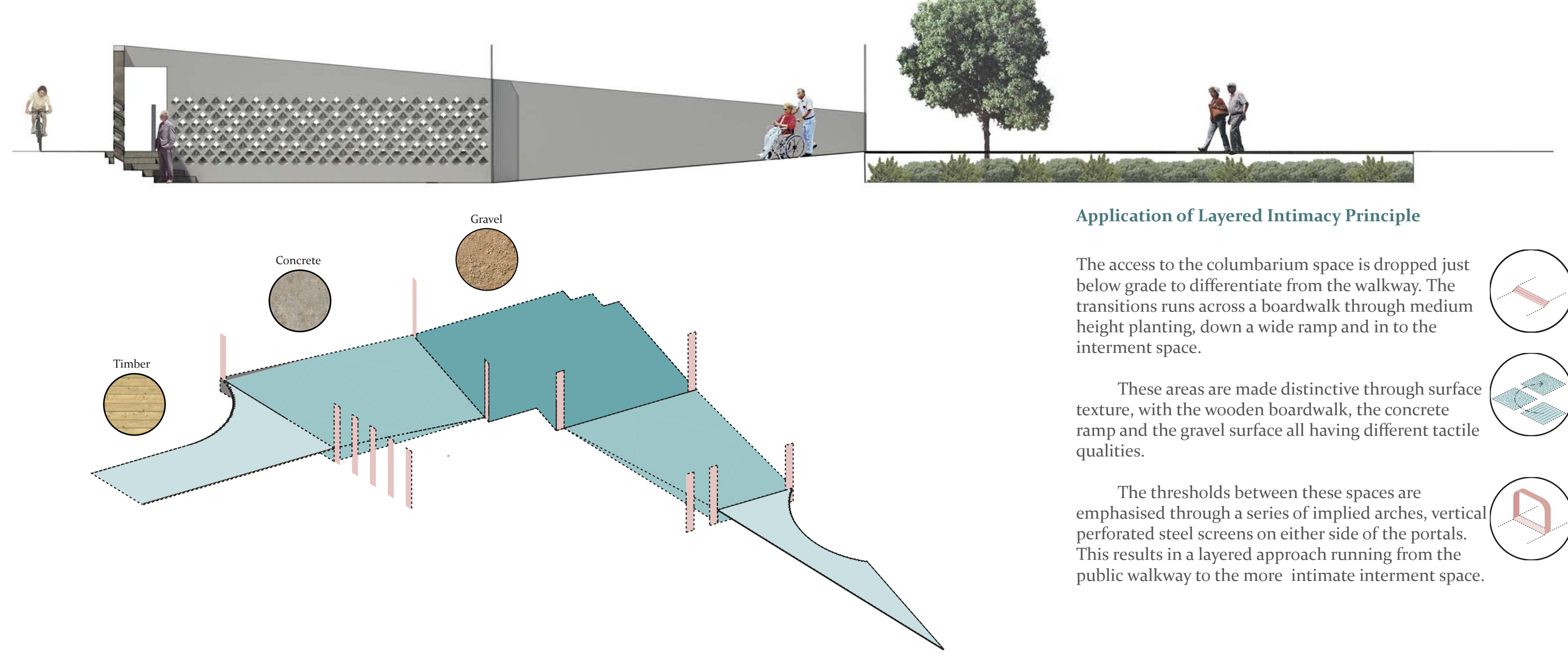

Application of Layered Intimacy Principle

The access to the columbarium space is dropped just below grade to differentiate from the walkway. The transitions runs across a boardwalk through medium height planting, down a wide ramp and in to the interment space.

These areas are made distinctive through surface texture, with the wooden boardwalk, the concrete ramp and the gravel surface all having different tactile qualities.

The thresholds between these spaces are emphasised through a series of implied arches, vertica perforated steel screens on either side of the portals. This results in a layered approach running from the public walkway to the more intimate interment space.
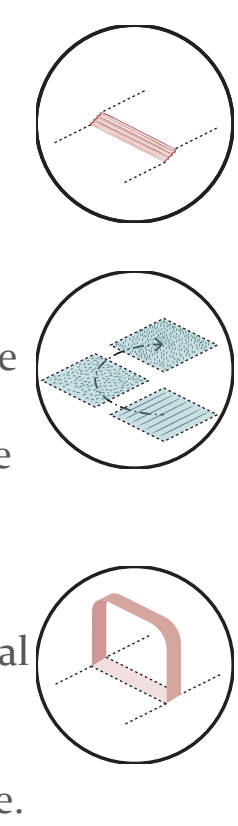


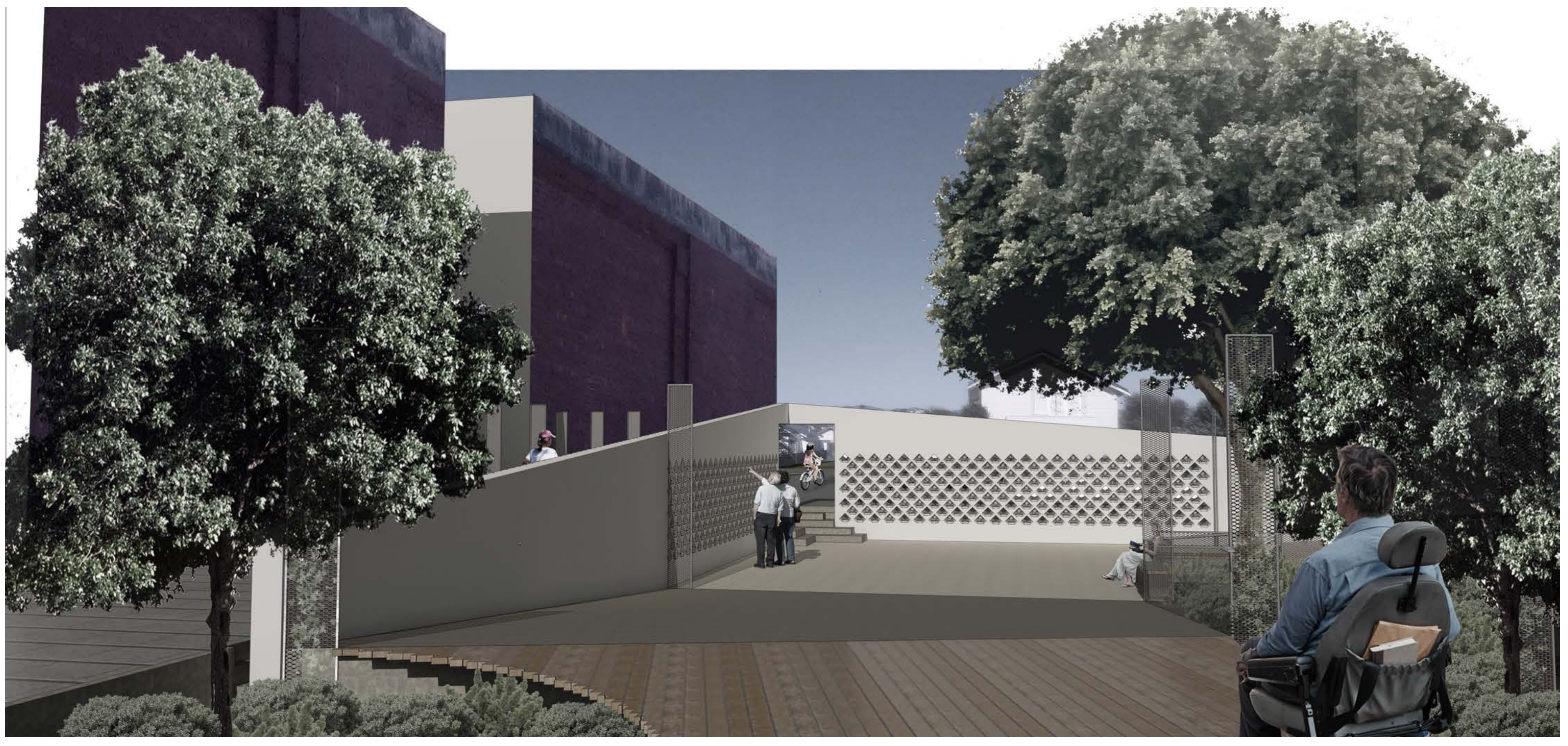




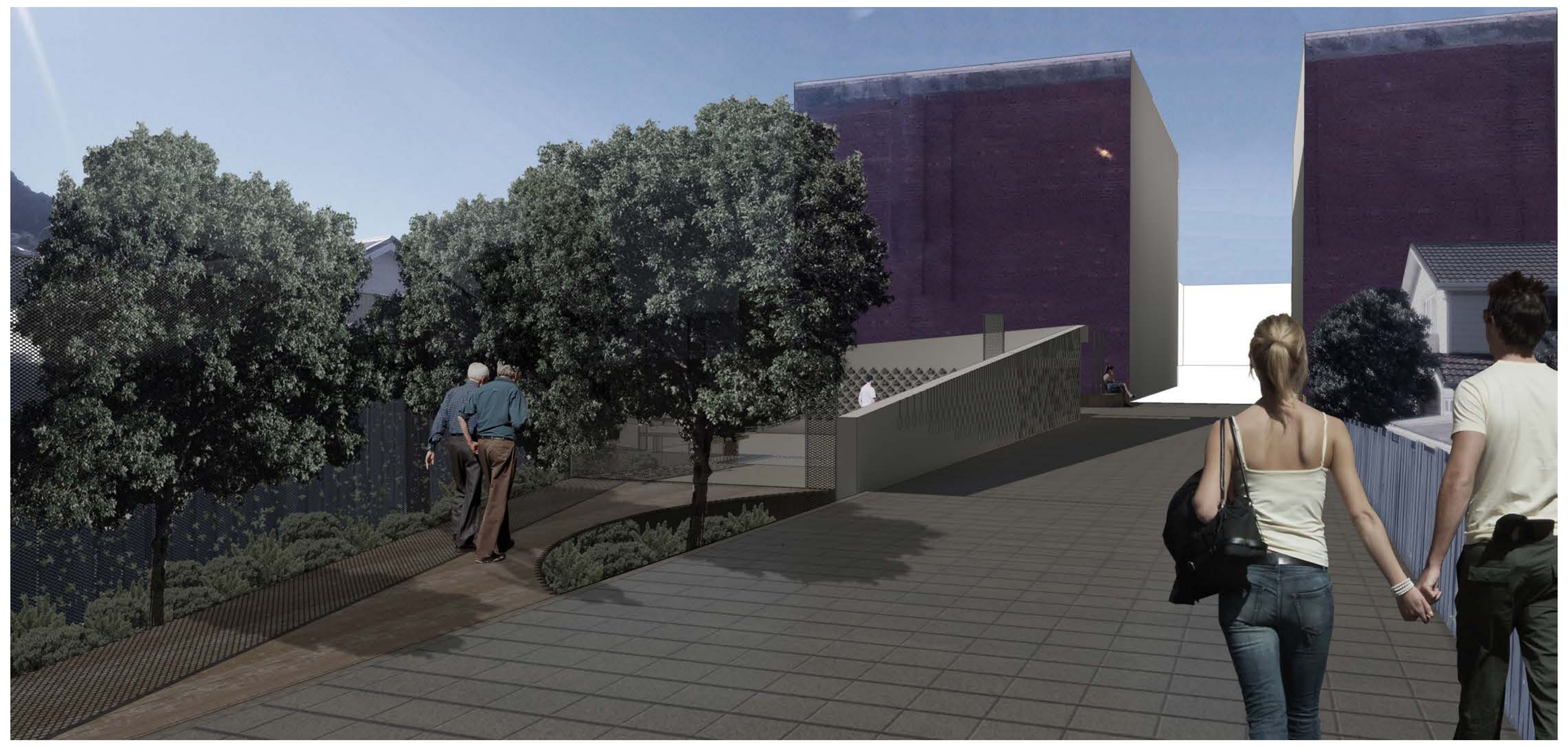





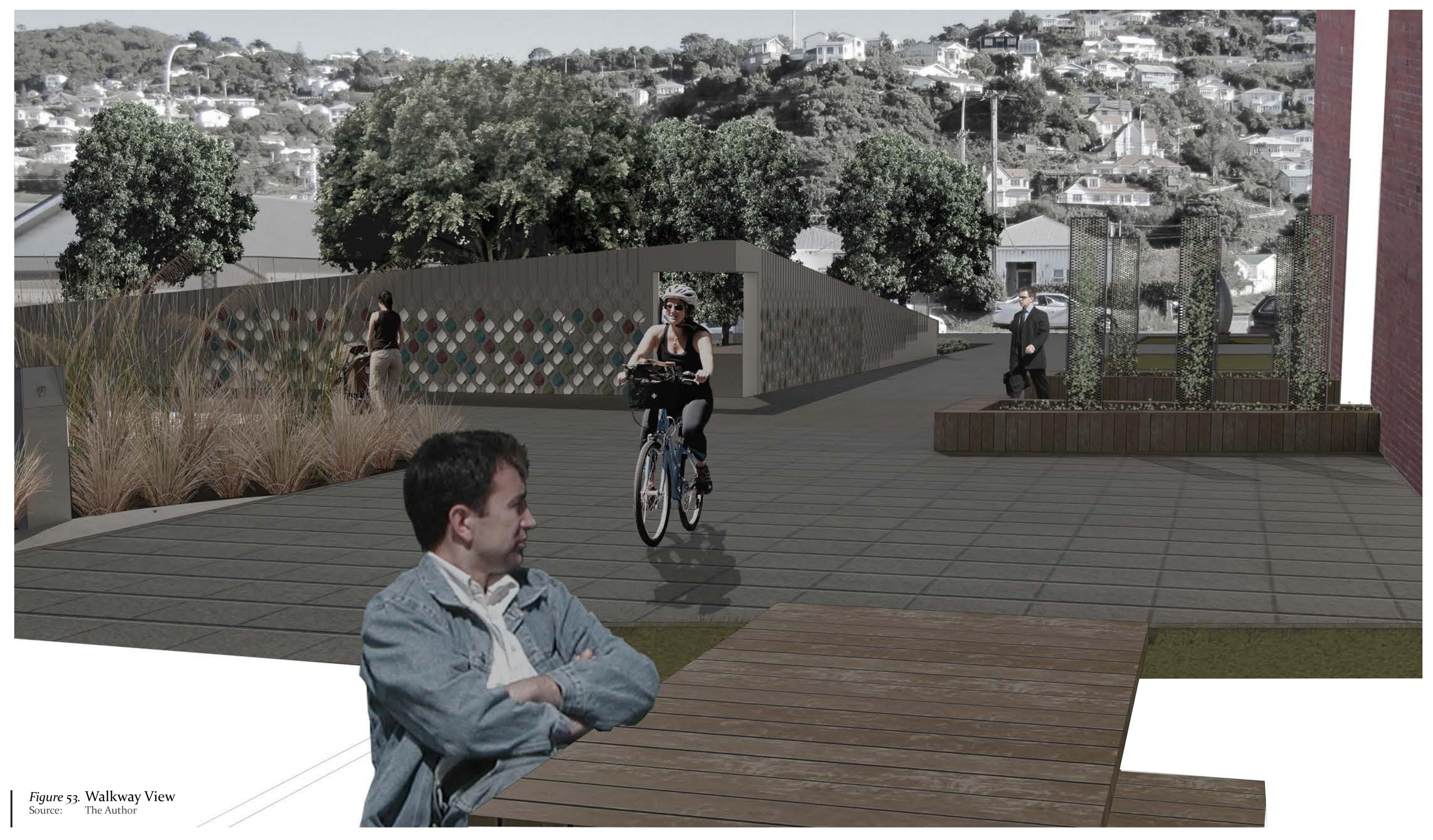




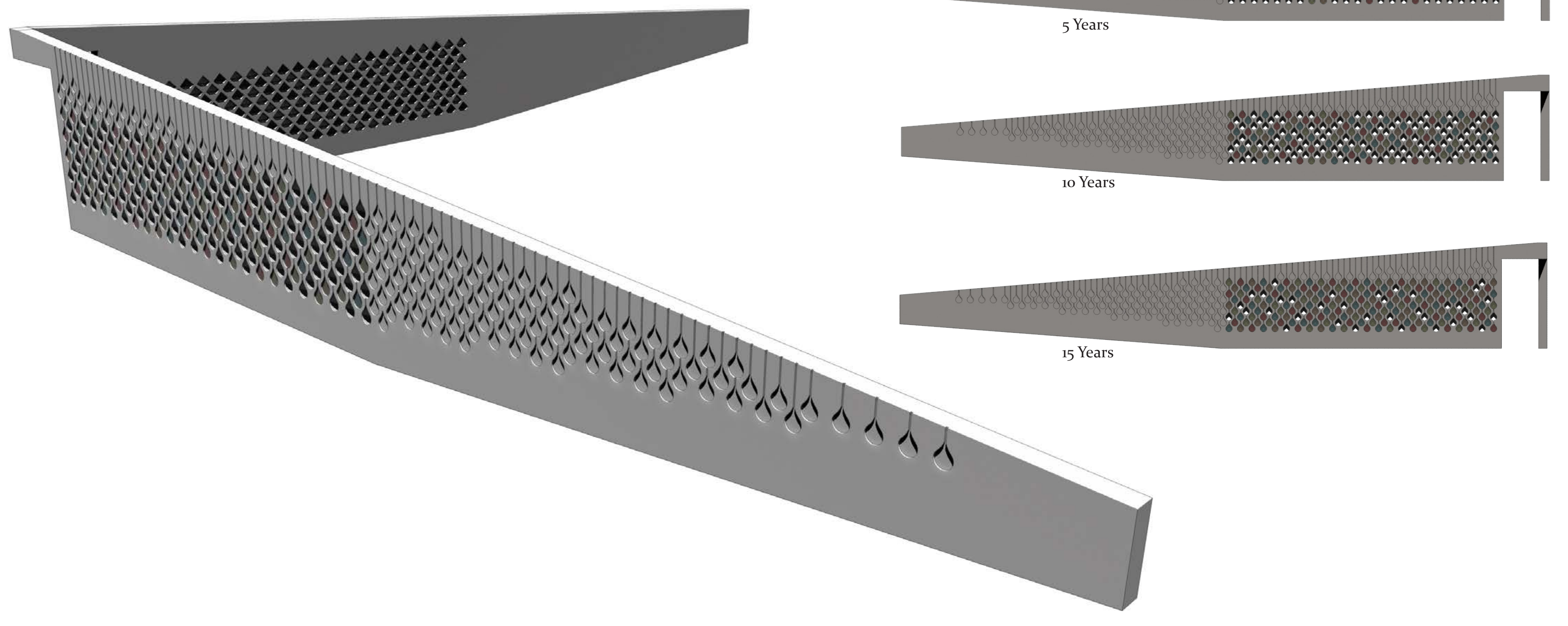




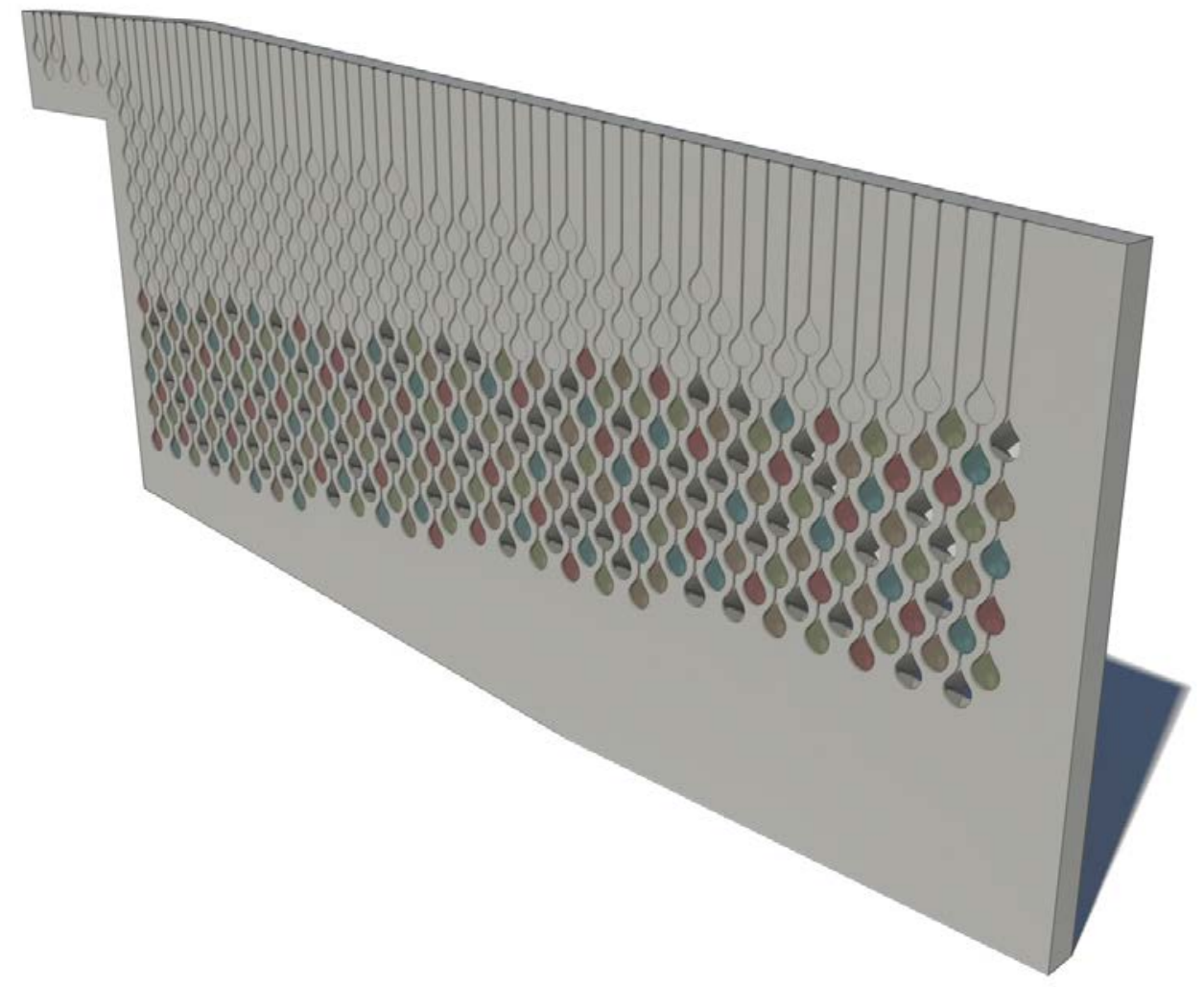

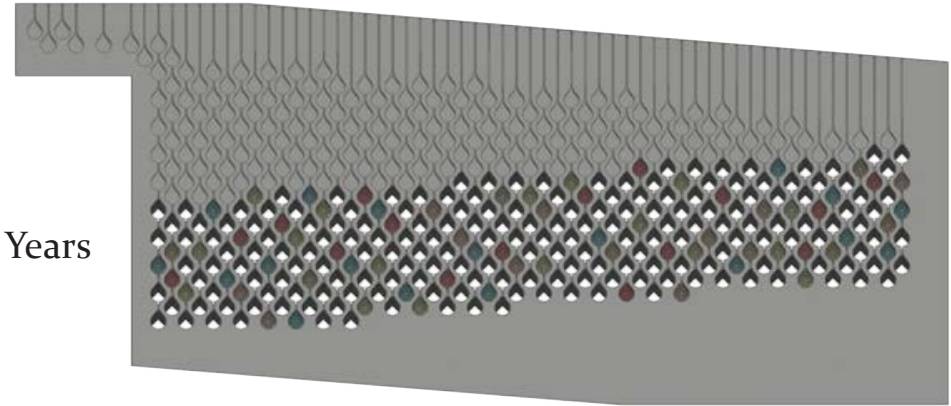

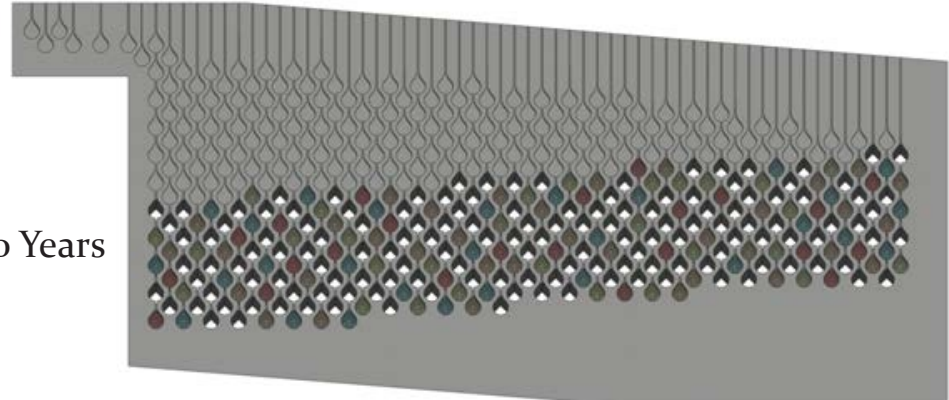

15 Years

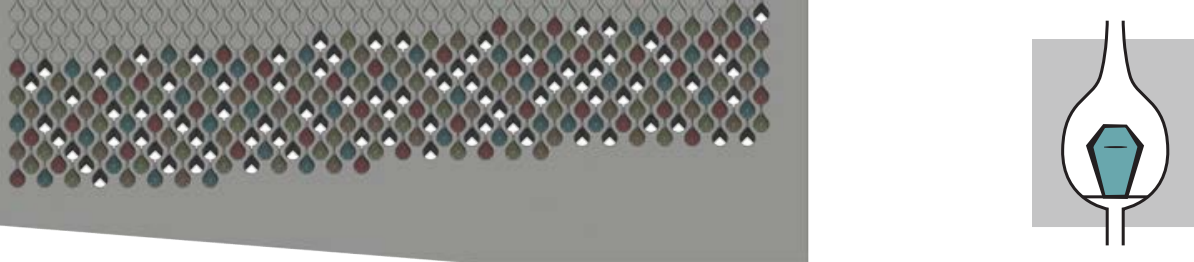

Application of Individualised Interment Principle

On both sites the walls effectively have a 'front' and 'back' end. The inward facing niches with engraved plaques and space for leaving flowers allow for traditional practices of commemoration to continue, while the outward facing multicoloured niche caps provide an option to individualise (through colour choice) the 'public face' of the interment.

This 'front' side of the wall does not immediately read as associated with commemoration. However the intrigue it sparks, with its steadily evolving mosaic, will lead to those who are

curious venturing to the other side and discovering the wall's true purpose.

This evolution over time extends beyond just the wall. In order to allow for the wall to be a sustainable interment option over time, niches will be leased for 25 year periods, and in vacating them families will be given the option of scattering the ashes in a plot near path with which each wall is associated. On this plot a native from each site's planting palette can be chosen by the family and planted, adding yet another layer of individualisation to the process.
Year 1

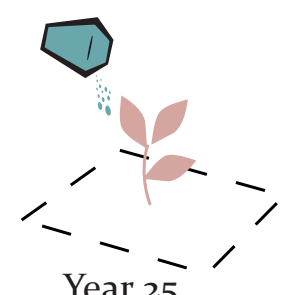

Year 25

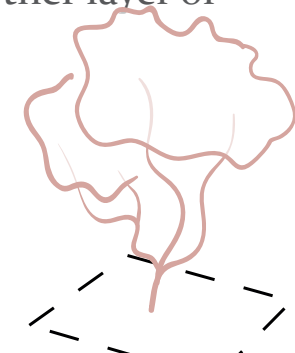

- 1 

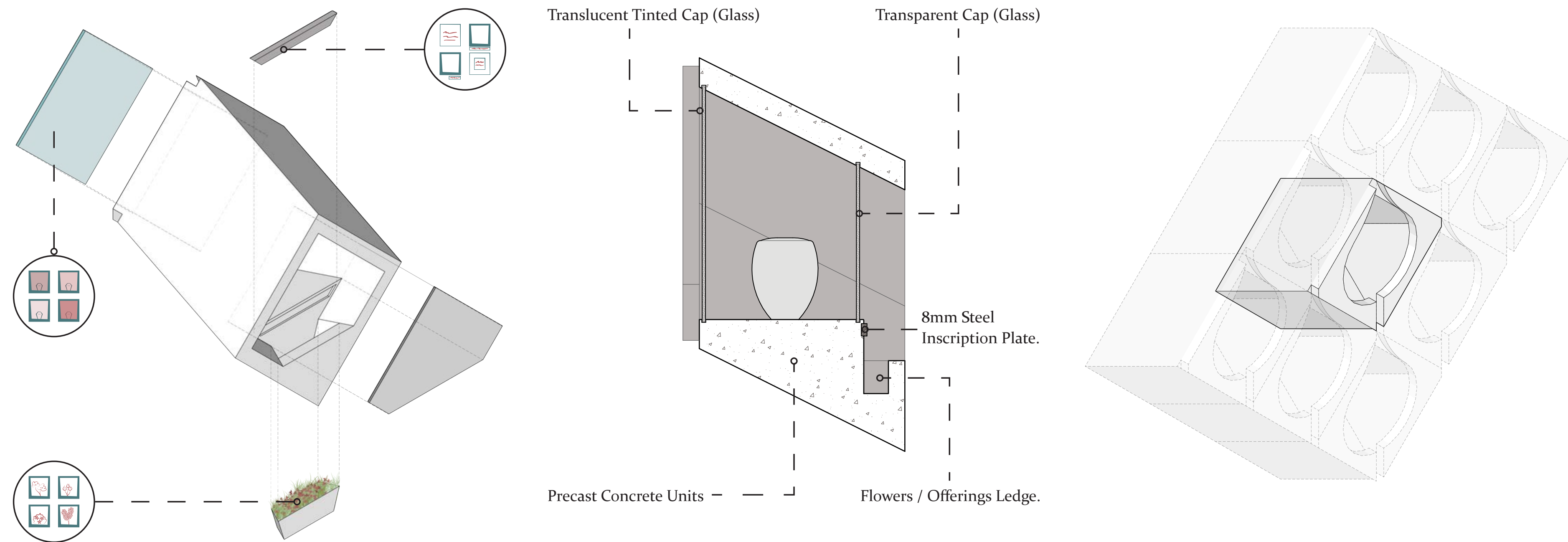

Precast Concrete Units - $\quad-\quad\lrcorner \quad$ Flowers / Offerings Ledge 


\section{Conclusions}

The design process executed in this chapter has led to the design of two spaces for the dead which break from many of

the current traditions in urban spaces for the dead, particularly when considered as a part of the larger network design from the previous chapter

Rather than sitting within existing large scale cemeteries they are nearby to the communities for whom they are built. They respond to this context by accommodating the various needs of the communities and mitigating their impact on the surrounding land uses and how those and uses might effect them. This is done by layering several distinctive spaces leading up to the columbarium, creating a transition from public to intimate.

These design case studies serve as examples of the design principles, particularly the final two, being implemented. They will be looked at as tests of the principles in the exegesis which follows, and will help to gauge their value. 



\section{EXEGESIS}

VII 



\section{Introduction}

n order to contribute to the discipline and the specific area of research of the thesis, its outputs need to be critically assessed.

This chapter seeks to reflect on both the design proposal for

Wellington City and the design principles which have served as its underlying structure.

This is achieved first through a more general review of the design, reflecting on some of the aspects which, while not directly related to the design principles, are none the less of significance. Next each of the five design principles are used to assess the design and the value of each principle is also reviewed.

Following this the relationships between these principles are reflected on. Finally a conclusion is made, visiting the implications of the thesis and the potential for further research in the area. 


\section{General Design Discussion}

Early investigations of the precedent case studies revealed that spaces for the dead, particularly columbaria (as upright opaque elements which often block views from multiple sides) often lack proper consideration of Crime Prevention Through Environmental Design (CPTED) issues. (Fig. 63 \& 64) As these precedent case studies were located within existing cemeteries, with gates that would close after hours, this was not a problem, however in transferring the tradition to the suburban core, such issues become much more important.

The first step to avoiding such issues was the choice of a single layered niche with openings on both sides, as is the case at Hörnli. Through this it is able to reduce areas of low visibility.

However avoiding CPTED issues has often caused issues, particularly in relation to the principle of Layered Intimacy, as intimacy effectively requires areas of low visibility. This has been approached by screening, both with vegetation and perforated steel fencing. In order to avoid entrapment spots, both walls have been given openings through to their front sides. (Fig 62) Howeve this does compromise the strong design integrity of the wall, particularly at the Brooklyn site where it serves as the viewing platform's 'end'.

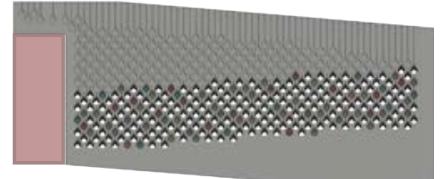

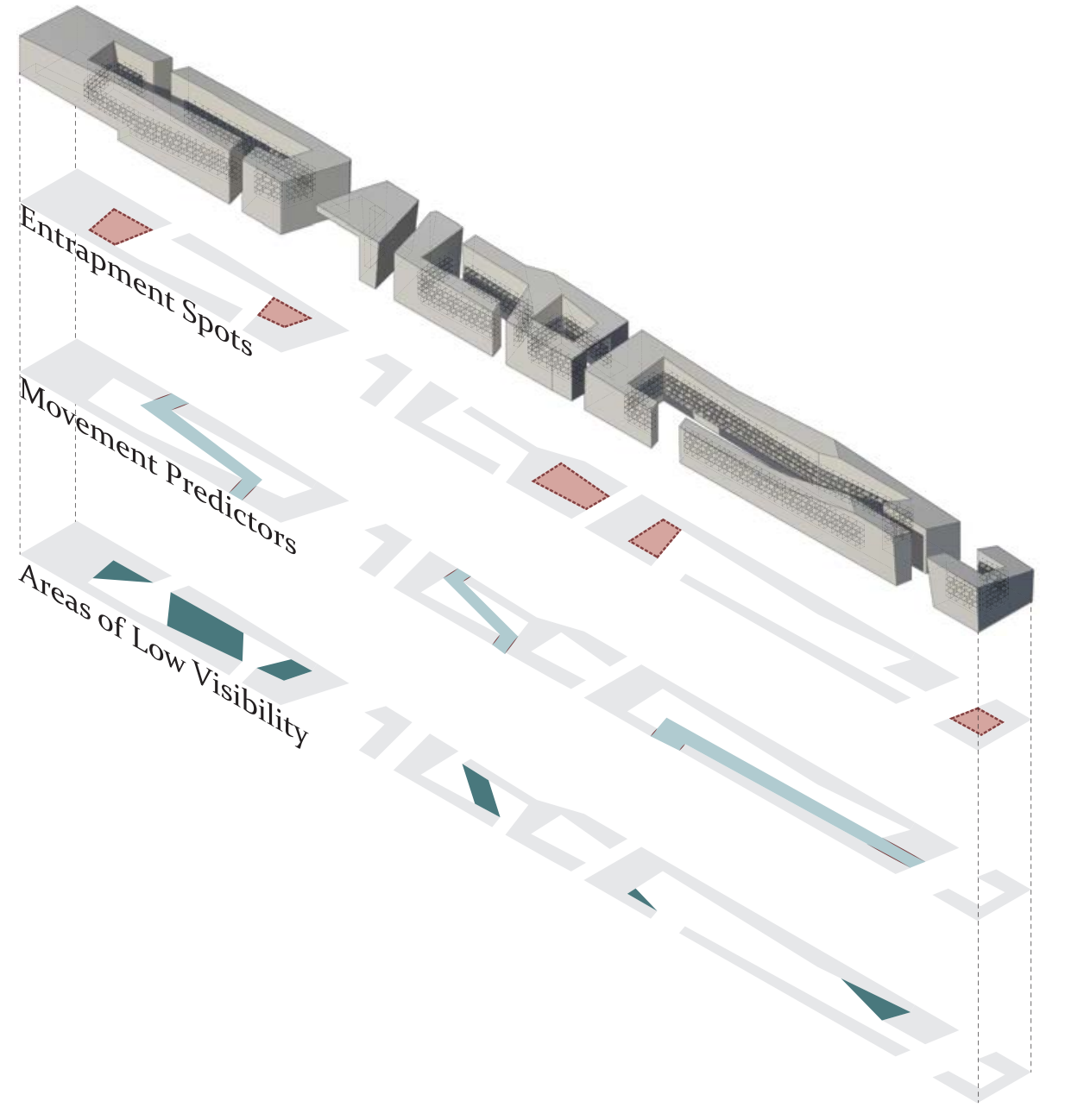

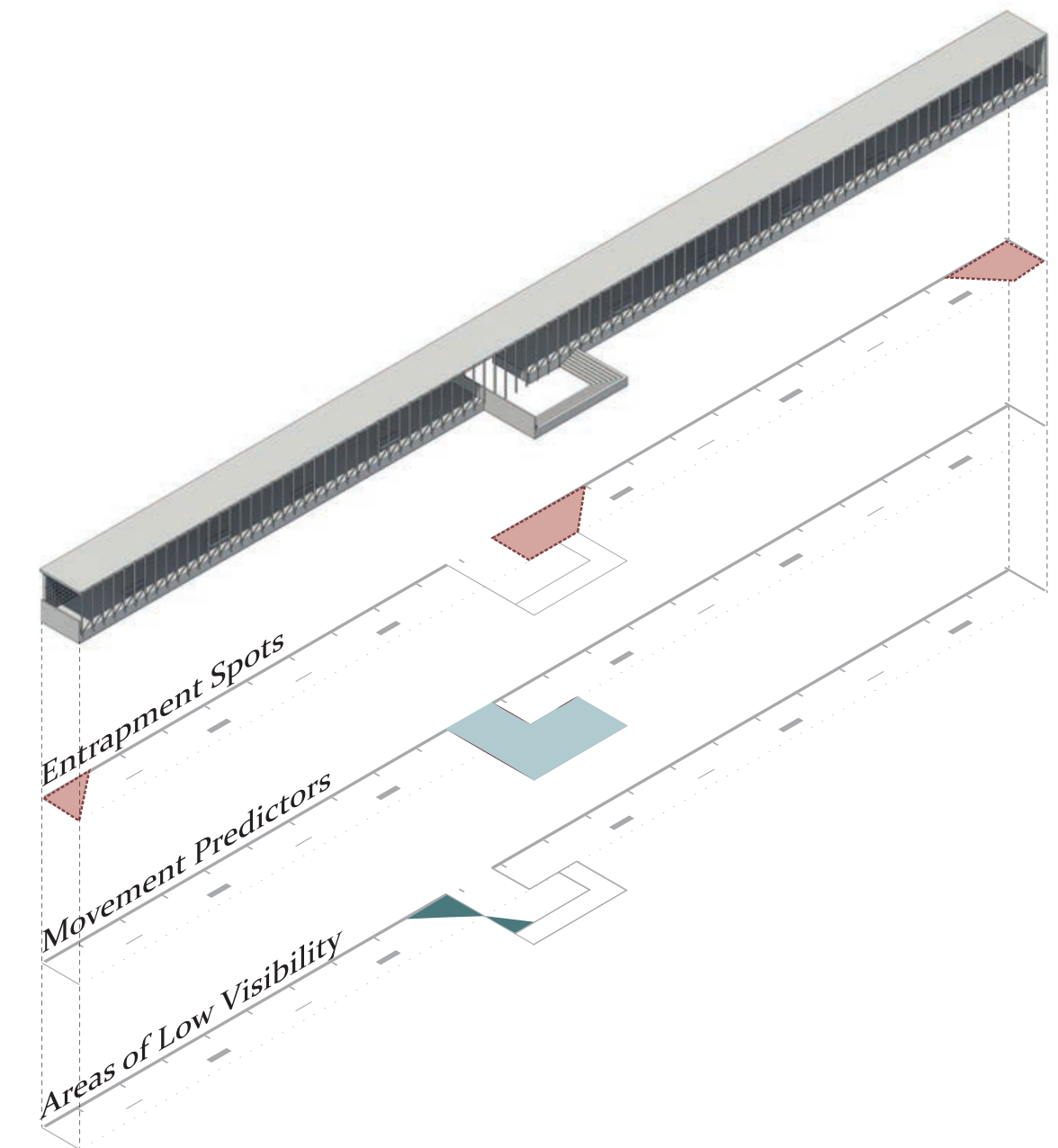

Figure 64. Hörnli CPTED Analysis 
One question is who would actually administer these spaces? This thesis has operated under the assumption that the network would be constructed and administered by the local council, as they the existing system, and are in fact bound by the Burial and Cremation Act to do so. However when considering the principles as a model for application across a wide variety of contexts, it is important to note that other models might also prove successful, particularly in other legislative contexts (outside of New Zealand).

The concept of a private trust, established by local government but held by the local community, might allow for a greater investment of confidence in the projects, and a sense of ownership of the public space. This would allow each suburb to develop a commemorative space which was tailored to their own needs.

Weather it is administered centrally (where rates would, at least in part, subsidise it) or through some other model, the economic sustainability of the columbarium would have to be ensured. One of the biggest issues, particularly in Wellington, is that currently plots are purchased with one lump sum, however the cemetery must be maintained in perpetuity. (Cemetery Management Plan 21) This means that older cemeteries effectively become money sinks. As few plots remain, very little money comes in, however maintenance costs are higher than ever, leading to an unstable economic equation.

It is for this reason that the niches of the columbarium would not be sold in perpetuity, but leased for a period of time. After 25 years the family would be given the option to renew the lease or transfer the ashes to a designated scattering / planting plot, adding another layer to the commemoration process while freeing the niche, allowing for greater economic sustainability.

However it does need to be acknowledged that such a system would likely serve as a slight culture shock to those used to perpetual interment. While people are more comfortable with the disinterment of cremated remains than that of buried ones, and scattering of ashes is already a widely practiced ritual, it is likely that this cultural shift would need to occur gradually. 


\section{Brooklyn}

As mentioned in the previous chapter, the Brooklyn site needs to serve as the beginning of the Moturoa stream, and in many ways this also makes it the northern entrance to Central Park (although an entran Brooklyn road). The design's two tiered staircase, which kinks at the stormwater exit which feeds the stream in order to create a connection between is intended to serve this function.

However this is not visible from street level, while the viewing platform is. The platform may spark intrigue which then leads people down to the stairs. However a more pronounced entrance may be necessary for the site to act as 'the entrance' rather than 'an entrance'. It is difficult to balance this programme which is effectively a third land use to be integrated) with the viewing platform and columbarium while allowing each to retain their integrity.

\section{Kilbirnie}

The easement over which the proposed community walkway runs is a likely source of controversy, as it is there to allow for sewerage from the city to be carried out to the treatment plant at Moa Point. While this fact is not well known and therefore unlikely to impact on people's perceptions of the site, it does create some issues.

The Kilbirnie Town Centre Plan states that any intervention on the easement would need to allow continued access to the pipes sitting below. (McIndoe et al. 26) However in order to provide the space necessary, the columbarium must encroach slightly on the easement. While it would not obstruct access to the pipes, as it extends only $1 / 3$ of the way on to the area, some alteration to the easement's border might be necessary, which might cause issues.

Unlike Brooklyn, Kilbirnie lacks shelter directly adjacen to the columbarium wall. This is not a necessity; the majority of the precedents were left open to the elements. However in Wellington's climate the inclusion of some form of shelter beyond the large Kanuka might be necessary.

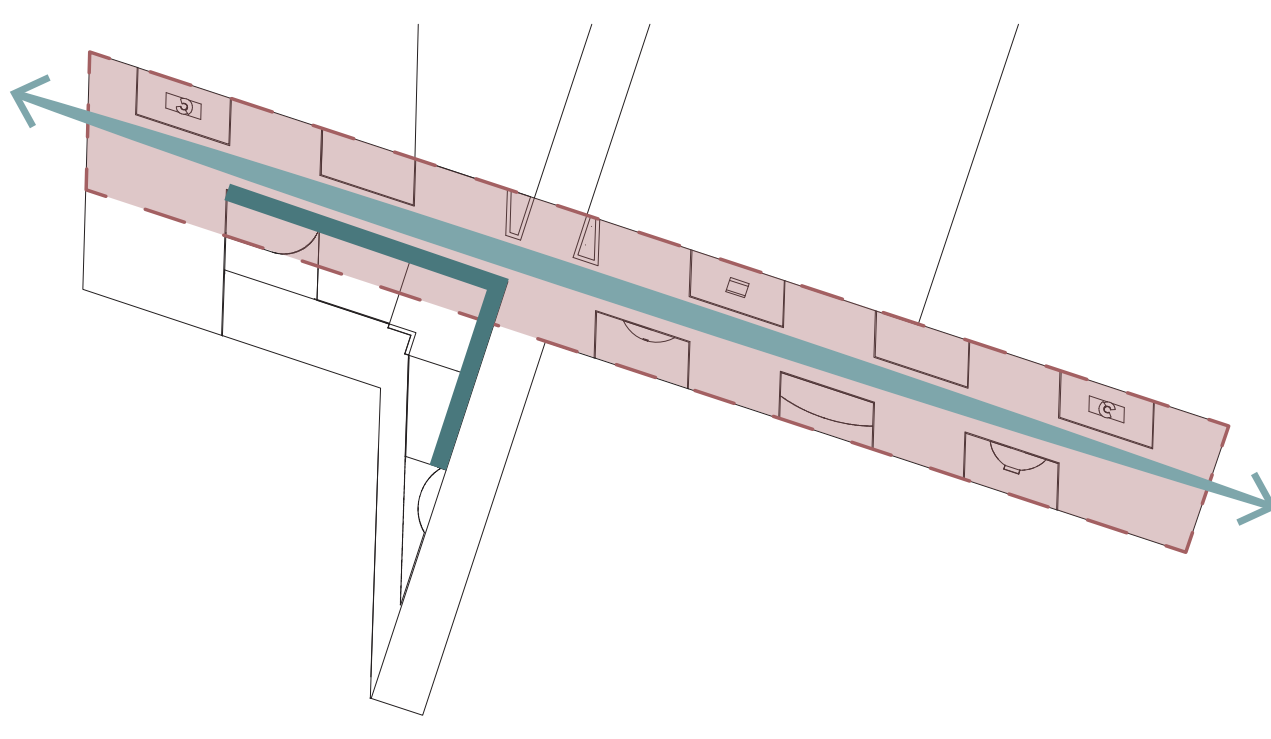

Easement Boundary

Pipe Location 


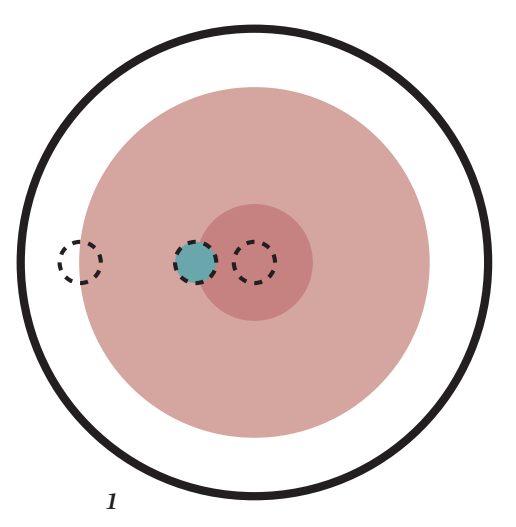

\section{Informed Introduction Discussion}

For Kilbirnie, which is in many ways a centre to several adjacent suburbs as well as itself, the core is arbitrarily constrained by council defined borders, which hardly fit with the principle's intended purpose, as the local community do not, for instance, make a distinction between Kilbirnie and Lyall Bay as a line in the ground. In many ways the selected site could be seen as an inter-suburb option catering to Kilbirnie, Rongatai and Lyall Bay, particularly considering the walkway will pass through all three.

In several cases sites which were selected within the core are later in the site selection process (while carrying out Obvious Improvement or Infill Impacts) found to be too close to the community's centre. While knowing the exterior limits of the core is important, it seems that also establishing a series of 'no go' zones may prevent redundant work assessing sites which are too close for comfort.

The layered analysis involved in the application of this principle helps to establish a 'ball park' area from which sites will be selected. It is important that the principle be kept in mind while carrying out the rest of site selections, and that the site's appropriateness in relation to the principle of an 'informed introduction' be considered from the city scale all the way to the parcel.

The simplification of roadways down to primary, secondary and tertiary allows for gaining a simplified understanding of the suburb at a large scale. However variables such as traffic volumes and pedestrian usage should also be known for major routes within the suburb. In particular, it could we worth investigating any situations where a primary road is actually serving predominantly as a thoroughfare for people from other areas. For instance the state highway to the north-east of Kilbirnie has been noted because, while it carries high volumes of traffic, few of these individuals will actually be from the local community. In some other cases during the site selection chapter, this issue was missed. Constable St in Newtown was seen as a primary road and created an extension of the 'core area', however in retrospect much of this use would come from those commuting from Kilbirnie and beyond. 


\section{Obvious Improvement Discussion}

While public stairways were identified as potential sites across a number of suburbs during the application of this principle, when the sites were examined at a closer scale access always became an issue. As such they may need to be omitted from the palette of underutilised spaces because, although they may present a development opportunity of some type, access is a necessity for a columbarium as many of the users do not have full mobility.

In some cases multiple land uses were added as a part of the clear benefit section of the principle. However in situations such as the viewing platform, columbarium and stormwater exit (and eventually park entrance) found in Brooklyn this becomes a little too complex. Rather than attempting to evenly distribute and integrate each of the land uses throughout the site, it may have been better to focus on two: the columbarium and the one of most obvious benefit, while allowing other land uses to become secondary programmes which, while still taken in to account, are no longer the design focus.

The techniques used in locating sites within the core may not align properly with the type of sites being sought. The sub

principle of underutilised spaces has lacked an experiential scale through the site selection process. These spaces are inherently the type of space one doesn't come across when examining from plan view, but rather something that is stumbled upon while walking the streets.

The two sub principles, while both working toward the same goal, go about it in very different ways and could almost be seen as separate principles both operating at similar scales. However by collecting the two together it does allow each to align specifically toward this goal, rather than being abstract principles with little relationship to the introduction of columbaria.

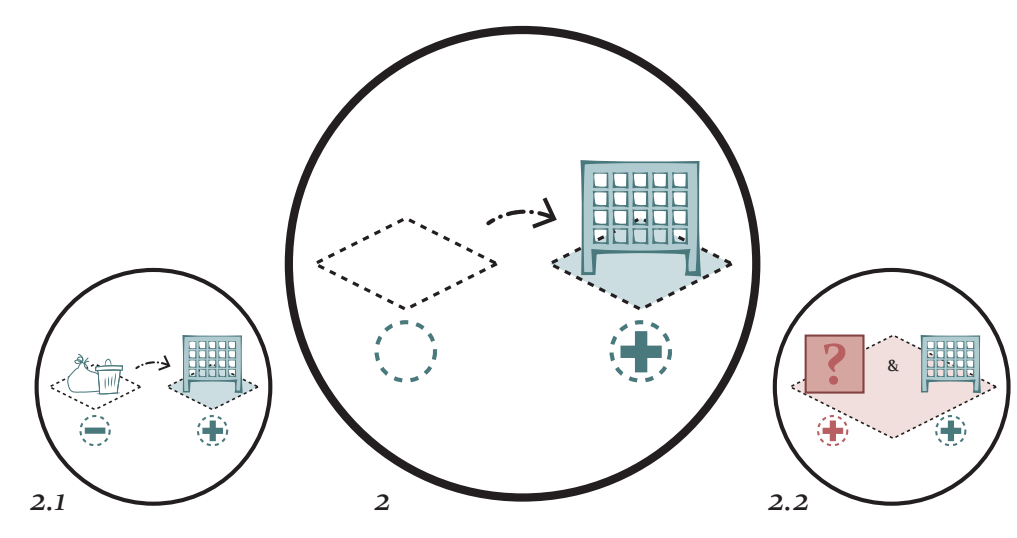




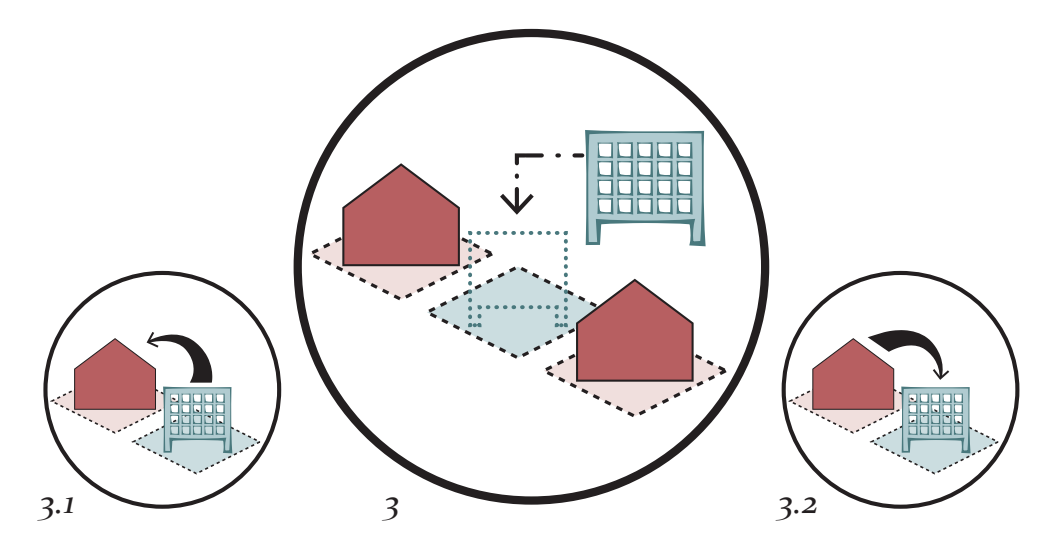

\section{Infill Impacts Discussion}

As almost every site is adjacent to a road, more effort to distinguish types of road may have allowed for a better

understanding of the potential impact on the site. While the

degree to which they would impact the site was identified during

visits and recorded during each site's Infill Impacts assessment,

had they been broken down in to different types of road prior to

this, such site by site assessment may not have been necessary.

On the Brooklyn site the apartments on the corner of

Brooklyn and Ohiro will, despite the mitigation provided by

the Kotukutuku, have a fairly strong visual connection to the

columbarium space. While this alleviates some CPTED concerns,

it does mean that the infill will have some impact on these

apartments.

With the residential development to the Kilbirnie site's North in the bus barn, the intention is for the columbarium space and walkway to occur prior to the establishment of 600 new dwellings. Even with this, the problem of reverse sensitivity of those moving in, plus the potential for the developers to take issue with the columbarium before breaking ground on their own project.
The potential for changes in societal attitudes surrounding death over time mean that the priorities of this principle will have to be malleable. While initially the focus will need to be on preventing effects by the spaces for the dead, if people do become more comfortable, this may shift to controlling the effects the space experiences in order to craft the best possible experience.

This also links to the fact that the principle tends to focus on the negative impacts, and how to mitigate them. There may be value in identifying potential positive impacts of a particular infill, and finding a way to amplify them.

While the application of this principle has been based almost entirely on adjacent land uses, there is an in between scale which has effectively been omitted: The parcel chosen will potentially have impacts and be impacted on not just adjacent land uses but on the 'block' or 'street' as a suburban entity. 


\section{Layered Intimacy Discussion}

In both designs the access ramps have been used as 'areas' However they are almost inherently transition spaces rather than true 'layers'. In Brooklyn they are slightly less transitional due to the perpendicular bands of planting and seating used to make each area distinctive, however in Kilbirnie they are serve only to funnel from the boardwalk spaces to the interment area. They are almost threshold areas between thresholds, and do not contribute to the layered intimacy principle as effectively as the distinctive areas which they link.

The Brooklyn gravel transition markers may not be entirely noticeable for the less haptic user. The surface threshold may need some form of augmentation. It is possible that the change from a slope to a landing and back again may serve this purpose.

The implied 'portals' may be obscured by the planting (in particular the Mahoe). The use of some horizontal element connecting the two 'pillars' might be necessary to prevent this. Perhaps even a simple wire with the Clematis trained along it.

This principle has been applied in what is still a fairly linear fashion, following Alexander's principles perhaps too closely.
Certainly the Brooklyn site is effectively a single sequence of spaces, while Kilbirnie does have two approaches, each echoing the same sequence. In many ways the principle could be altered to allow for a more 'onion skin' style of layering where the areas are bands wrapping around a core space which can be approached from a range of sides, allowing for a less linear spatial sequence.

In many ways the outer layers are all there simply to be passed through, rather than functional areas in their own right. It may be necessary to assign some specific programme to these a disposing of old 作 space for interment.

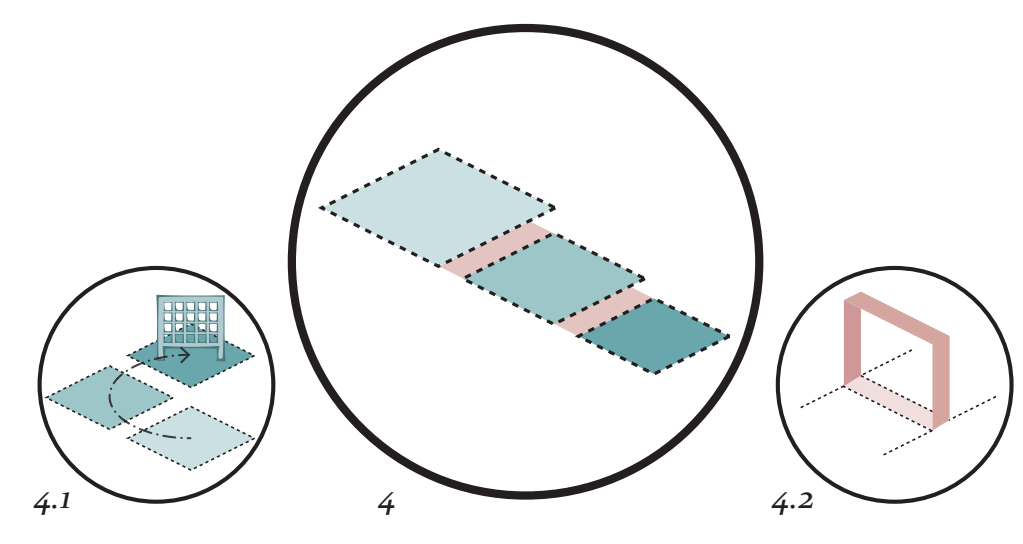




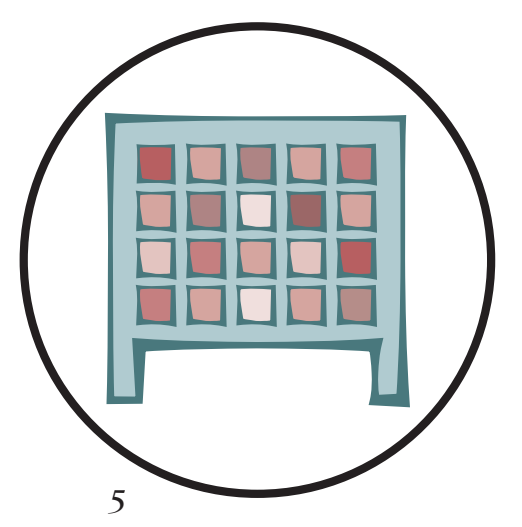

\section{Individualised Interment Discussion}

During the initial few years the wall will be relatively underpopulated', meaning that it may lack in texture. It might be necessary to implement 'placeholder' caps which help to spark interest in the wall, or even having the caps in place from the beginning. However this would limit the extent to which the community would begin to associate the 'front' of the wall with certain individuals, because the introduction of a new urn would no longer be a noticeable action.

It is certainly important to provide multiple ways of individualising interments, not just one. An individual is likely to have a number of people who might visit their memorial. Each of these people will perceive this individual differently and it is important that they have the opportunity to express themselves through the option of their choice, rather than forcing just one on them.

The principle of individualised interment could also be applied at the city scale. By allowing each suburb to individualise their wall, either through the trust model described in the general design discussion or alternatively by designing the wall to be somehow 'open source', to make some sort of formal response to the commemorative practices of that particular suburb. This would allow the columbarium to individualise not only to the person, but also to the community as a whole. 


\section{Synthesis of Principles}

The Informed Introduction principle is both the largest scale principle, and the one which is most key to the aim of the thesis. Because of this, while it is applied early within site selection, it is also taken into consideration throughout the whole process.

While assessing potential sites through the infill impacts principle at the scale of several parcels, the concept of this site's ocation with relation to the suburbs 'core' and the location of the community's centre should still be accounted for:

While the Infill Impacts principle is primarily applied during the site selection process, certain impacts identified through the principle must then be mitigated. As such Infill Impacts also needs to be worked in to the design process. Both the Obvious Improvement and Layered Privacy principles all allow for this.

The added value section of the Obvious Improvement principle, when carried out on site, seeks to align the purpose of the columbarium with that of the other land use. This mitigates the impacts of the columbarium on surrounding land uses by masking' the purpose of the site.
Though Layered Privacy the impacts of surrounding land uses can be filtered so that they do not affect the inner interment space. The outer layers, through planting, screens and level changes can begin to 'absorb' the publicness of the surround environment, allowing for the inner area to maintain a more intimate one.

There is an inherent conflict between the principle of Layered Intimacy and the underutilised spaces section of Obvious Improvement. As most of the underutilised spaces are relatively small parcels, often with awkward shapes and slopes, the ability to establish the sequence of spaces is required for a Layered Privacy.

This is particularly true if Alexander's notion of a sequence running from larger spaces to smaller ones is followed. There is effectively a minimum space that needs to be provided adjacent to the columbarium wall in order to accommodate commitment ceremonies as well as general commemorative activity. If each area leading up to this one must be larger, then the exterior layer is likely to be too large to be accommodated in most of the underutilised spaces identified throughout the site selection process.
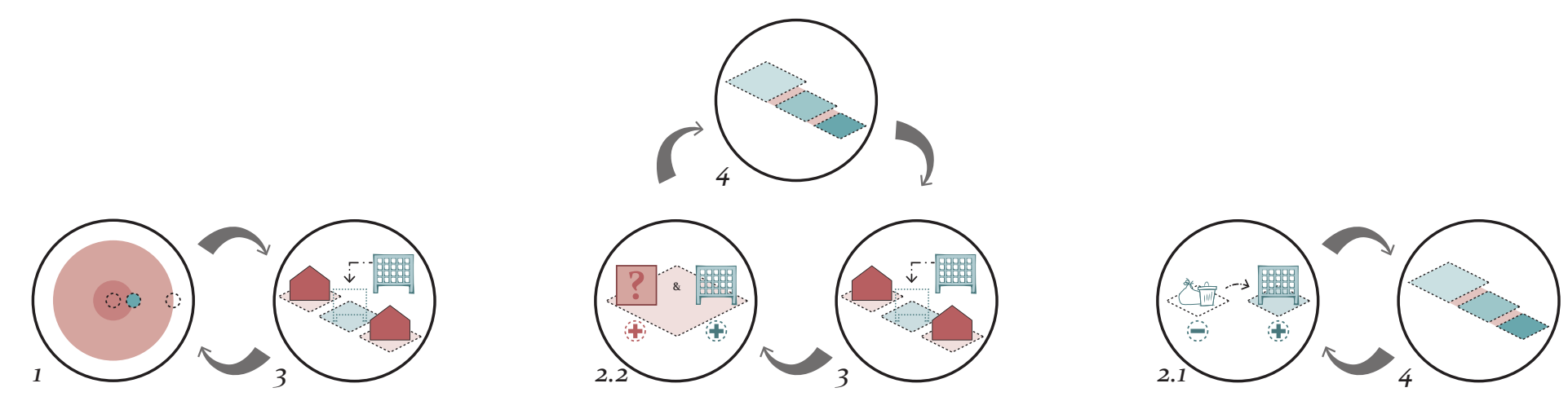


\section{Conclusions}

The words 'find' and 'space' are perhaps the most important ones within the stated aim in the introduction to this thesis:

\section{To find space for the dead within today's suburbs.}

'Find' because the place where this land use should go is not, like with many other land uses, explicit or subject to some best practice.

This is effectively why these spaces have been pushed to the periphery, because no system has been in place to find space for them.

'Space' because, particularly with columbaria, exactly how to assemble and compose the wall and the spaces associated with it is relatively under-investigated. The concept of a columbarium as a new 'type' of space for the dead is almost universally unfamiliar and requires careful development.

The principles developed through the research in this thesis have allowed for an investigation of this aim, first at an abstract contextual level and then through a more specific design test applying them to Wellington City. While there are certainly overlaps the site selection process is effectively a response to the 'find' while the design responds to the 'space'.

\section{Implications}

There is the potential for the Design Principles to be refined through further research, and to be tested through further design case studies. Their application to cities with different contexts and demographics would further highlight their strengths and weaknesses.

The goal is that these principles might also be of help for practitioners looking to realise such a scheme, or carry out some design involving a new type of space for the dead.

With this said, the wider topic of space for the dead through the lense of spatial design disciplines is still extremely underdeveloped. This thesis has focused on suburbs, but while the majority of urban dwellers now live in such places, there are a significant number who also chose to live in the city centre. a significant nutre. with a city's centre, rather than a suburbs, certainly warrants investigation.

Populations have also become more transient, with people moving, on average, every 10 years this. While this thesis investigates a location specific space for the dead, the question of how a population with no strong connection to a single suburb, city or even country might be provided with a space for the dead.

As this thesis focuses almost entirely on culturally nonspecific options, there is still room for investigation into networks which begin to provide for multiple cultural contexts. In particular, when the country has an endemic spatial tradition: the Urupa (or Maori burial ground), that has barely been investigated from a spatial design perspective, it is important that such research occur.

Ultimately this thesis investigates one of many possible models for the distribution and composition of spaces for the dead. However as an augmentation to an existing system, with a series of design principles as an explicit underlying structure, this model allows for finding space for the dead in today's suburbs. It is a step towards suburban populations having a greater awareness of their own mortality, and one would hope, a better appreciation of life. 



\section{List of Figures}

\section{INTRODUCTION}

\section{Figure 1. Problem Diagram}

Figure 2. Aim Diagram

Figure 3. Thesis Structure Diagram

\section{Context Review}

Figure 4. Matrix of Religious/Cultural Mortality Traditions

\section{Precedents}

Figure 5. De Nieuwe Ooster Regional Context

Figure 6. Columbarium Aerial Photograph

Figure 7. Columbarium Plan and Section

Figure 8. Columbarium Analysis

Figure 9. Friedhof Hörnli Regional Context

Figure 11. Columbarium Photograph

Figure 10. Columbarium Plan and Section

Figure 12. Columbarium Analysis

Figure 13. Friedhof Steckborn Regional Context

Figure 14. Columbarium Photograph

Figure 15. Columbarium Plan and Section

Figure 16. Columbarium Analysis

Figure 17. Westminster Urban Columbarium Regional Context 32

Figure 18. Columbarium Photograph

Figure 19. Columbarium Plan and Section

Figure 20. Columbarium Analysis

Figure 21. Cimetière Arradon Regional Context

Figure 22. Columbarium Photograph

Figure 23. Columbarium Plan and Section

Figure 24.

Design PRinciples

Figure 25. Core Identification Example

\section{Site Selection}

\section{Figure 26. Existing Network Plan}

Figure 27. Suburban Centre to Cemetery Elevations

Figure 28. Phasing of Columbarium Network

Figure 29. Completed Network Plan

Figure 30. Brooklyn Suburban Core Identification

Figure 31. Additional Suburban Core Identifications

Figure 32. Brooklyn Potential Site Identification

Figure 33. Additional Suburbs Site Identification

Figure 34. Brooklyn Infill Impact Assessments

Figure 35. Additional Suburb's Infill Impact Assessment

Figure 36

Proposed Sites \& Programme

DESIGN

Figure 37

Figure 38

Figure 39.

Figure 40.

Figure 41

Figure 42

Figure 43.

Figure 44.

Figure 45

Figure 46.

Figure 47.

Figure 48.

Figure 49.

Figure 50.

Figure 51

Figure 52
Figure 53.

Figure 54.

Figure 55.

Figure 56.

Brooklyn Niche Occupation Progression

Figure 58. Long Term Niche Occupation

Figure 59. Niche Explosion

Figure 60. Niche Section

Figure 61.

Columbarium

EXEGESIS

Figure 62. Kilbirnie and Brooklyn CPTED Considerations

Figure 63. Nieuwe Ooster CPTED Analysis

Figure 64. Hörnli CPTED Analysis

Figure 65. Hörnli CPTED Analysis 


\section{Works Cited}

"Karres En Brands: De Nieuwe Ooster Columbarium, Amsterdam, the Netherlands 2004-2008." A + U: architecture and urbanism.4(475) (2010): 88-91. Print.

"National Guidelines for Crime Prevention through Environmental Design in New Zealand.” Ed. Justice, Ministry of. Wellington: Ministry of Justice, 2005. Print.

“Wellington Consolidated Bylaw 2008." Ed. Council, Wellington City2008. Print.

Alexander, C. A Pattern Language: Towns, Buildings, Construction. Oxford University Press, USA, 1977. Print.

Archinect. "Showcase: Westminster Presbyterian Church: Urban Columbarium and Courtyards". 2009. (May 29 2009): 17 November 2012. <http://archinect.com/features/ article/89230/showcase-westminster-presbyterian-churchurban-columbarium-and-courtyards $>$.

ASLA. "2009 Professional Awards - Westminster Presbyterian Church Fellowship Courtyard and

Memorial Columbarium,Minneapolis, Mn”. 2009. 17 November 2012. <http://www.asla.org/2009awards/349.html>.

Basmajian, Carlton, and Christopher Coutts. "Planning for the Disposal of the Dead." Journal of the American Planning Association 76.3 (2010): 305-17. Print.

Brennan, A. "The Office of Unsolicited Architecture: The Golden Gate Remains: Bridging the Deficits." Volume 14 (2007): 5261. Print.
Brett, Cate Honore. "Review of the Burial and Cremation Act 1964". Wellington, 2010. The Law Commission. 15 September 2012. <http://www.lawcom.govt.nz/project/ review-burial-and-cremation-act-1964>.

Council, Wellington City. Cemetery Management Plan. Wellington2010. Print.

Curl, J.S. A Celebration of Death. London: Batsford, 1993. Print.

Forum, Architectural. "Living Memorials." Architectural forum 83 (1945): 141-41. Print.

Freud, Sigmund. "Our Attitude Towards Death." Thoughts for the Times on War and Death. 1915. Vol. 12. Th E Pelican Freud Library. Ringwood: Penguin, 1985. Print.

Grainger, Hilary J. "Golders Green Crematorium and the Architectural Expression of Cremation.” Mortality 5.1 (2000): 53 - 73. Print.

Harnik, Peter, and Aric Merolli. "Cemeteries Alive - Graveyards Are Resurging as Green Spaces for the Public." Landscape Architecture 100.12 (2010): 44-50. Print.

Harvey, Sarah. "Funeral Fireworks... Why Kiwis Go out with a Bang." Sunday Star Times (2012). 13 September $2012<$ http:// www.stuff.co.nz/national/6867468/Funeral-fireworks-whyKiwis-go-out-with-a-bang>.

Hudnut, J. The Monument Does Not Remember. 1945. Print.

Madec, Philippe. “Cementary, Extension, Arradon”. Rennes. 28 January 2013. <http://www.atelierphilippemadec.com/ landscaping/cementeries/cementary-extension.html>.
McIndoe, Graeme, et al. Kilbirnie Town Centre Plan. Wellington: Wellington City Council, 2009. Print.

McIntosh, J. Ancient Mesopotamia: New Perspectives. ABC-CLIO 2005. Print.

Morgan, Scott. "More Cemetery Sites Planned." Western Leader 10 March 2011 2011. Print.

Morris, I. Burial and Ancient Society: The Rise of the Greek CityState. Cambridge University Press, 1990. Print.

Mumford, Lewis. “"Monuments and Memorials"” Good Housekeeping 120.17 (1945): 106-08. Print.

Neal, Tracy. "Plan Considers Ageing Population." Nelson Mail 26 September 2012 2012. Print.

Odland, Aaron. "Urban Spaces - Reconnecting with the Urban Dead - a Traveling Scholar Studies How Cemeteries Fit into the Urban Fabric." Landscape Architecture 100.6 (2010): 4049. Print.

paris.fr. "Cimetière Père-Lachaise". Paris. paris. fr. 21 May 2012. <http://equipements.paris.fr/ Cimeti\%C3\%A8re_P\%C3\%A8re-Lachaise .

Parliamentary, Library. "New Zealand's Aging Population." (2011). 10 October $2012<$ http://www.parliament.nz/en-NZ/ ParlSupport/ResearchPapers/2/b/8/ooPlibCIPo31-NewZealand-s-ageing-population.htm>. 
Rainey, Reuben M. “The Garden as Narrative: Lawrence Halprin’s Franklin Delano Roosevelt." Places of Commemoration: Search for Identity and Landscape Design. Ed. WolschkeBulmahn, Joachim. Washington, DC: Dumbarton Oaks Research Library and Collection, 2001. 377 - 416. Print.

Raudon, Sally. "Angelboxes in the Land of Ikea." Funeralcare 34 (2012): 29-30. Print.

Contemporary Funeral Practices. 2011. Radio. Walbridge, Catherine, 2o September 2011.

The NZ Way of Death. 2012. Radio. Scott, Richard, 19 May 2012.

Retief, FP. "Burial Customs and the Pollution of Death in Ancient Rome: Procedures and Paradoxes." Acta theologica 26.2 (2010): 128 - 46. Print.

Rugg, Julie. "Defining the Place of Burial: What Makes a Cemetery a Cemetery?” Mortality 5.3 (2000): 259-75. Print.

---. “'A Few Remarks on Modern Sepulture': Current Trends and New Directions in Cemetery Research." Mortality 3.2 (1998): 111-28. Print.

---. "Lawn Cemeteries: The Emergence of a New Landscape of Death." Urban History 33.02 (2006): 213-33. Print.

Sachdeva, Sam. "Sydenham Locals 'Not Keen' on Crematorium." The Press 2011. Print.

Saibene, Gianluca. "Gianluca Saibene: Ampliamento Del Cimitero Communale, Giussano, Italia, 2001." Casabella 66.705 (2002): 80-[85]. Print.
Schwass, Margot. Last Words: Approaches to Death in New Zealand's Cultures and Faiths. Wellington: Bridget Williams Books, 2005. Print.

Shanken, Andrew M. "Planning Memory: Living Memorials in the United States During World War Ii." The Art Bulletin 84.1 (2002): 130-47. Print.

Sokol, David. "Making an Exit: As Cremation Gains Acceptance Worldwide, Architects Are Laying to Rest the Overly Dignified Crematorium Designs of the Past - Instead, They're Firing up Their Imaginations, Creating Sublime Modernist Places for Saying Farewell." Azure 23.179 (2007) 79-85. Print.

Stock, Rob. "Are We Too Poor to Die?" Stuff (2012). 10 Octobe $2012<$ http://www.stuff.co.nz/business/money/6867599/ Are-we-too-poor-to-die $>$.

Tarlow, S. Bereavement and Commemoration: An Archaeology of Mortality. John Wiley \& Sons, 1999. Print.

Torres-Rouff, Christina, William J. Pestle, and Blair M. Daverman. "Commemorating Bodies and Lives at Kish's 'a Cemetery': (Re)Presenting Social Memory." Journal of Social Archaeology 12.2 (2012): 193-219. Print.

Vetschpartner. "The Hörnli Cemetery”. 2011. Landzine. 23 January 2013. <http://www.landezine.com/index.php/2011/o1/theho\%CC\%88rnli-cemetery-by-vetschpartner-landscapearchitecture/>.
Vogt, G. Miniature and Panorama: Vogt Landscape Architects, Projects 2000-2012. Lars Müller Publishers, 2011. Print.

---. "Steckborn Cemetery, Steckborn". 2010. 17 November 2012. $<$ http://www.vogt-la.com/en/node/244/typology>.

Wilford, John Noble. "At Ur, Ritual Deaths That Were Anything but Serene "The New York Times (2009). 3 October 2012 <http://www.nytimes.com/2009/10/27/science/27ur.html? $\mathrm{r}=\mathrm{O}>$.

Wolschke-Bulmahn, Joachim, ed. Places of Commemoration: Search for Identity and Landscape Design. Washington, DC: Dumbarton Oaks Research Library and Collection, 2001. Print.

Worpole, Ken. Last Landscapes: The Architecture of the Cemetery in the West. London: Reaktion, 2003 Print. 
